\title{
The Mechanism of Potassium tert-Butoxide-Catalyzed Ketones Hydrogenation in Solution-Phase
}

Pavel A. Dub* and Nikolay V. Tkachenko

Chemistry Division, Los Alamos National Laboratory, Los Alamos, New Mexico 87545, United States

Cartesian coordinates and energy data for the optimized structures

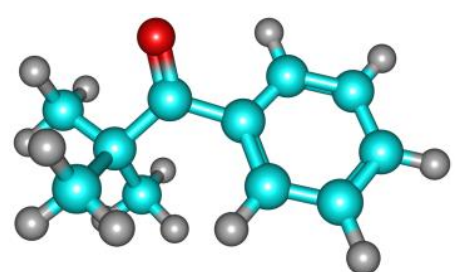

basis = def2-QZVP

$E=-502.848970647$

$G=-502.692015($ Temperature $=483.15$ pressure $=39.64)$

$\mathbf{G}=\mathbf{- 5 0 2 . 6 5 9 3 9 4}($ Temperature $=\mathbf{2 9 8 . 1 5}$ pressure $=\mathbf{2 4 . 4 5})$

$\begin{array}{llll}6 & 0.733842000 & 0.684269000 & -0.275455000\end{array}$

$\begin{array}{llll}8 & 0.952317000 & 1.798947000 & -0.702765000\end{array}$

$\begin{array}{llll}6 & -0.699231000 & 0.253496000 & -0.135528000\end{array}$

$\begin{array}{llll}6 & 1.898947000 & -0.224313000 & 0.129975000\end{array}$

$\begin{array}{llll}6 & -1.150317000 & -1.047192000 & -0.351752000\end{array}$

$\begin{array}{llll}6 & -1.623247000 & 1.244500000 & 0.197560000\end{array}$

$\begin{array}{llll}6 & -2.968048000 & 0.940351000 & 0.329335000\end{array}$

$6 \quad-2.501291000 \quad-1.345018000 \quad-0.245414000$

$\begin{array}{llll}6 & -3.410529000 & -0.356412000 & 0.103229000\end{array}$

$1-0.464280000 \quad-1.832918000 \quad-0.625933000$

$\begin{array}{llll}1 & -2.841887000 & -2.353551000 & -0.432973000\end{array}$

$1 \quad-4.460752000 \quad-0.595439000 \quad 0.196889000$

$\begin{array}{lll}-3.670722000 & 1.714485000 & 0.603841000\end{array}$

$\begin{array}{lll}-1.273952000 & 2.254205000 & 0.360840000\end{array}$

$\begin{array}{lll}1.566557000 & -1.069784000 & 1.362229000\end{array}$

$\begin{array}{lll}2.255739000 & -1.119964000 & -1.067328000\end{array}$

$\begin{array}{lll}3.107877000 & 0.656788000 & 0.448027000\end{array}$

$\begin{array}{lll}0.802977000 & -1.818499000 & 1.168915000\end{array}$

$\begin{array}{lll}2.891995000 & 1.339108000 & 1.270373000\end{array}$

$\begin{array}{lll}2.496445000 & -0.515272000 & -1.941571000\end{array}$

$\begin{array}{lll}2.467772000 & -1.590488000 & 1.686229000\end{array}$

$\begin{array}{lll}1.226862000 & -0.443041000 & 2.187793000\end{array}$

$\begin{array}{lll}3.944265000 & 0.022488000 & 0.740003000\end{array}$

$\begin{array}{lll}3.409606000 & 1.247547000 & -0.413635000\end{array}$

$\begin{array}{lll}3.134275000 & -1.713269000 & -0.811533000\end{array}$

$\begin{array}{lll}1.457064000 & -1.807264000 & -1.336381000\end{array}$

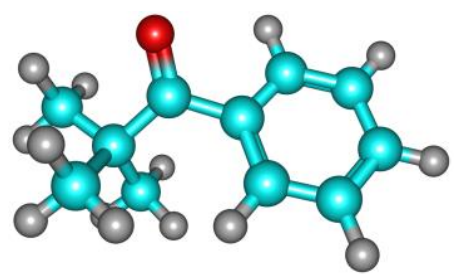

basis = def2-QZVP

$E=-502.835874537$ (gas)

$\mathbf{G}=\mathbf{- 5 0 2 . 6 8 5 1 3 6}$ (Temperature $=\mathbf{4 8 3 . 1 5}$, gas)

$6 \quad 0.728598000 \quad 0.714795000-0.184906000$

$\begin{array}{lllll}8 & 0.937039000 & 1.869927000 & -0.467249000\end{array}$

$\begin{array}{llll}6 & -0.705949000 & 0.255595000 & -0.089529000\end{array}$
$6 \quad 1.909982000$

$6-1.136604000$

$6-1.658042000$

$-3.002929000$

$-2.486586000$

$-3.421354000$

$-0.433090000$

$-2.806011000$

$-4.471502000$

$-3.726044000$

$-1.318442000$

1.676188000

2.148590000

3.158312000

0.865375000

3.033450000

2.334647000

2.585352000

1.451962000

4.012834000

3.366529000

3.030233000

1.317157000

$-0.229728000$

$-1.060463000$

1.249722000

0.936826000

$-1.372563000$

$-0.377586000$

$-1.854136000$

$-2.396020000$

$-0.624711000$

1.717456000

2.269639000

$-1.083156000$

$-1.109374000$

0.625793000

$-1.796614000$

1.284183000

$-0.488687000$

$-1.640904000$

$-0.452872000$

$-0.024969000$

1.247642000

$-1.727741000$

$-1.770841000$

0.088032000

$-0.241284000$

0.137192000

0.225503000

$-0.172001000$

0.067945000

$-0.430426000$

$-0.305130000$

0.130002000

0.412688000

0.240139000

1.339840000

$-1.149790000$

0.308785000

1.229729000

1.166794000

$-2.025384000$

1.563606000

2.200472000

0.491225000

$-0.557427000$

$-0.980375000$

$-1.376643000$

\section{H $-H$}

basis = def2-QZVP

$E=-1.16927069945$

$G=-1.174636($ Temperature $=483.15$ pressure $=39.64$ )

$\mathbf{G}=\mathbf{- 1 . 1 6 7 5 5}($ Temperature $=\mathbf{2 9 8 . 1 5}$ pressure $=\mathbf{2 4 . 4 5})$

$1 \quad 0.000000000 \quad 0.000000000 \quad 0.369453000$

$\begin{array}{llll}1 & 0.000000000 & 0.000000000 & -0.369453000\end{array}$

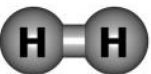

basis = def2-QZVP

$E=-1.16950926726$ (gas)

$\mathbf{G}=\mathbf{- 1 . 1 8 0 4 2 6}$ (Temperature $=\mathbf{4 8 3 . 1 5}$, gas)

$\begin{array}{llll}1 & 0.000000000 & 0.000000000 & 0.368715000\end{array}$

$\begin{array}{llll}1 & 0.000000000 & 0.000000000 & -0.368715000\end{array}$

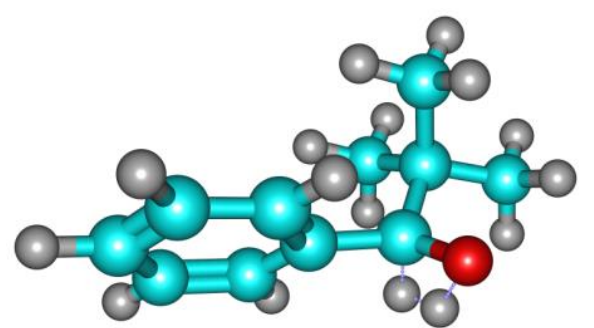

basis = def2-QZVP

$E=-503.925617078$ 


$\begin{array}{lrrr}\mathbf{v}=\mathbf{1 7 8 4} \mathbf{~ c m}^{-1} & & \\ \mathbf{G}=\mathbf{- 5 0 3 . 7 5 3 8 7 5} \text { (Temperature=483.15 pressure=39.64) } \\ \mathbf{G}=\mathbf{- 5 0 3 . 7 2 0 9 1 9} \text { (Temperature=298.15 pressure=24.45) } \\ 1 & 0.928876000 & 0.589645000 & -1.673560000 \\ 6 & -1.348512000 & -1.171480000 & 0.225140000 \\ 6 & -0.678132000 & -0.122976000 & -0.394143000 \\ 6 & -1.354688000 & 1.057949000 & -0.672724000 \\ 6 & -2.691308000 & 1.195273000 & -0.325075000 \\ 6 & -3.354061000 & 0.155397000 & 0.312557000 \\ 6 & -2.680229000 & -1.027356000 & 0.587067000 \\ 6 & 0.787111000 & -0.328920000 & -0.722885000 \\ 8 & 1.096148000 & -1.544341000 & -1.237995000 \\ 6 & 1.807378000 & 0.253975000 & 0.283342000 \\ 1 & -0.825049000 & -2.098119000 & 0.415274000 \\ 1 & -0.842116000 & 1.870279000 & -1.172892000 \\ 1 & -3.214233000 & 2.113077000 & -0.554939000 \\ 1 & -4.394561000 & 0.262647000 & 0.585672000 \\ 1 & -3.194582000 & -1.843699000 & 1.074709000 \\ 1 & 1.099054000 & -0.273265000 & -1.965819000 \\ 6 & 3.205114000 & 0.142557000 & -0.320469000 \\ 1 & 3.933963000 & 0.583528000 & 0.358673000 \\ 1 & 3.480223000 & -0.894535000 & -0.496436000 \\ 1 & 3.262548000 & 0.680118000 & -1.270304000 \\ 6 & 1.717233000 & -0.606952000 & 1.545176000 \\ 1 & 2.498545000 & -0.299558000 & 2.240245000 \\ 1 & 0.756412000 & -0.487182000 & 2.045036000 \\ 1 & 1.863864000 & -1.661200000 & 1.313442000 \\ 6 & 1.511371000 & 1.712076000 & 0.624558000 \\ 1 & 1.516833000 & 2.340940000 & -0.268494000 \\ 1 & 0.549804000 & 1.828355000 & 1.121741000 \\ 1 & 2.283569000 & 2.086441000 & 1.296355000 \\ 6 & & & \\ 6 & & \end{array}$

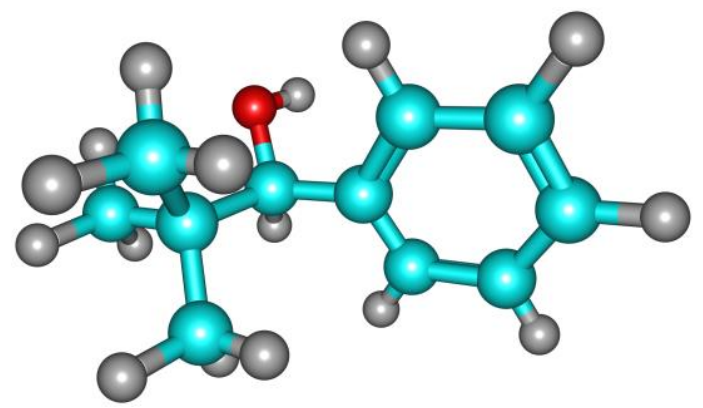

basis = def2-QZVP

$E=-504.058993140$

$\mathbf{G}=\mathbf{- 5 0 3 . 8 8 0 2 4 7}($ Temperature $=\mathbf{4 8 3 . 1 5}$ pressure $=\mathbf{3 9 . 6 4}$ )

$\mathbf{G}=\mathbf{- 5 0 3 . 8 4 6 8 7 9}($ Temperature $=\mathbf{2 9 8 . 1 5}$ pressure $=\mathbf{2 4 . 4 5}$ )

$\begin{array}{llll}0.944964000 & 0.444059000 & -1.68850\end{array}$

$\begin{array}{llll}6 & -1.293893000 & -1.124486000 & 0.281498000\end{array}$

$\begin{array}{llll}6 & -0.660770000 & -0.097016000 & -0.413723000\end{array}$

$\begin{array}{llll}6 & -1.393886000 & 1.040800000 & -0.739703000\end{array}$

$\begin{array}{llll}6 & -2.725037000 & 1.161071000 & -0.365371000\end{array}$

$\begin{array}{llll}6 & -3.345242000 & 0.135152000 & 0.335054000\end{array}$

$\begin{array}{llll}6 & -2.626383000 & -1.009254000 & 0.654071000\end{array}$

$\begin{array}{llll}6 & 0.794522000 & -0.203307000 & -0.817478000\end{array}$

$8 \quad 1.135923000-1.540099000-1.161406000$

$\begin{array}{llll}6 & 1.793734000 & 0.273658000 & 0.262532000\end{array}$

$\begin{array}{llll}1 & -0.741147000 & -2.020632000 & 0.528501000\end{array}$

$\begin{array}{llll}1 & -0.916741000 & 1.838295000 & -1.295612000\end{array}$

$\begin{array}{llll}1 & -3.279606000 & 2.051487000 & -0.627992000\end{array}$

$\begin{array}{llll}1 & -4.383534000 & 0.223299000 & 0.623323000\end{array}$

$\begin{array}{llll}1 & -3.104439000 & -1.815728000 & 1.193085000\end{array}$

$\begin{array}{llll}1 & 0.518542000 & -1.850950000 & -1.830477000\end{array}$

$\begin{array}{llll}6 & 3.207375000 & 0.155215000 & -0.311315000\end{array}$

$\begin{array}{llll}1 & 3.931370000 & 0.561623000 & 0.395726000\end{array}$

$\begin{array}{llll}1 & 3.473150000 & -0.881432000 & -0.509349000\end{array}$

$\begin{array}{llll}1 & 3.297650000 & 0.714686000 & -1.244351000\end{array}$

$\begin{array}{llll}6 & 1.690176000 & -0.565779000 & 1.534569000\end{array}$

$\begin{array}{llll}1 & 2.458863000 & -0.252034000 & 2.242158000\end{array}$

$\begin{array}{llll}1 & 0.722749000 & -0.441302000 & 2.020830000\end{array}$

$\begin{array}{llll}1 & 1.838821000 & -1.625075000 & 1.326768000\end{array}$

$\begin{array}{llll}6 & 1.520768000 & 1.739311000 & 0.596381000\end{array}$

$\begin{array}{llll}1 & 1.558835000 & 2.364142000 & -0.298012000\end{array}$ $\begin{array}{llll}1 & 0.546553000 & 1.874181000 & 1.065385000\end{array}$

$\begin{array}{llll}1 & 2.278409000 & 2.103989000 & 1.290685000\end{array}$

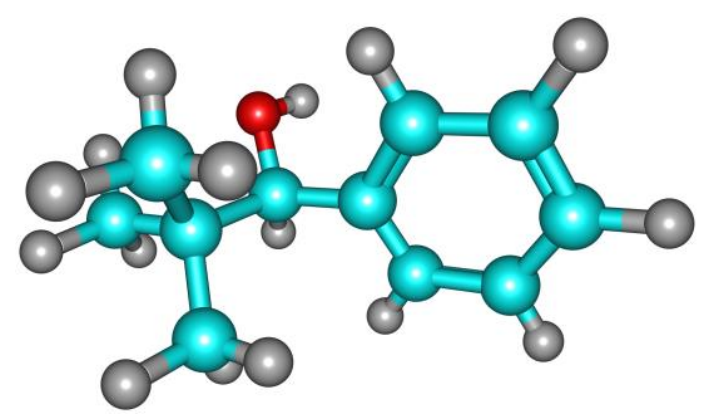

basis = def2-QZVP

$E=-504.045754275$ (gas)

$\mathbf{G}=\mathbf{- 5 0 3 . 8 7 1 2 3 1}$ (Temperature $=\mathbf{4 8 3 . 1 5}$, gas)

$\begin{array}{llll}1 & 0.948048000 & 0.453410000 & -1.688441000\end{array}$

$\begin{array}{llll}6 & -1.290565000 & -1.123634000 & 0.272112000\end{array}$

$\begin{array}{llll}6 & -0.660811000 & -0.089374000 & -0.414781000\end{array}$

$\begin{array}{llll}6 & -1.396733000 & 1.047772000 & -0.732608000\end{array}$

$\begin{array}{llll}6 & -2.727254000 & 1.161345000 & -0.358036000\end{array}$

$\begin{array}{llll}6 & -3.343875000 & 0.128768000 & 0.333874000\end{array}$

$\begin{array}{llll}6 & -2.622605000 & -1.015092000 & 0.644654000\end{array}$

$\begin{array}{llll}6 & 0.794339000 & -0.195971000 & -0.817418000\end{array}$

$\begin{array}{llll}8 & 1.138416000 & -1.530325000 & -1.154227000\end{array}$

$\begin{array}{llll}6 & 1.794998000 & 0.276164000 & 0.262925000\end{array}$

$\begin{array}{llll}1 & -0.728481000 & -2.015716000 & 0.511543000\end{array}$

$\begin{array}{llll}1 & -0.921176000 & 1.850846000 & -1.281295000\end{array}$

$\begin{array}{llll}1 & -3.284369000 & 2.051336000 & -0.613691000\end{array}$

$\begin{array}{llll}1 & -4.381784000 & 0.211922000 & 0.622326000\end{array}$

$\begin{array}{llll}1 & -3.098286000 & -1.825817000 & 1.178020000\end{array}$

$1 \quad 0.495981000-1.862409000-1.784357000$

$\begin{array}{llll}6 & 3.205924000 & 0.152130000 & -0.317218000\end{array}$

$\begin{array}{llll}1 & 3.937546000 & 0.522249000 & 0.400888000\end{array}$

$\begin{array}{llll}1 & 3.442773000 & -0.882554000 & -0.551967000\end{array}$

$\begin{array}{llll}1 & 3.303109000 & 0.740570000 & -1.230929000\end{array}$

$\begin{array}{llll}6 & 1.686587000 & -0.577836000 & 1.525287000\end{array}$

$\begin{array}{llll}1 & 2.458524000 & -0.280611000 & 2.235246000\end{array}$

$\begin{array}{llll}1 & 0.719917000 & -0.450577000 & 2.011029000\end{array}$

$\begin{array}{llll}1 & 1.824335000 & -1.632642000 & 1.293450000\end{array}$

$\begin{array}{llll}6 & 1.523714000 & 1.739679000 & 0.607380000\end{array}$

$\begin{array}{llll}1 & 1.566959000 & 2.370565000 & -0.282372000\end{array}$

$\begin{array}{llll}1 & 0.547423000 & 1.870536000 & 1.071723000\end{array}$

$\begin{array}{llll}1 & 2.279848000 & 2.097785000 & 1.305616000\end{array}$

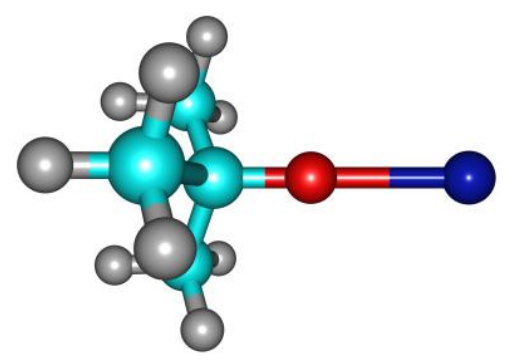

basis = def2-QZVP

$E=-833.053457844$

$\mathbf{G}=\mathbf{- 8 3 2 . 9 8 5 7 4 0}($ Temperature $=\mathbf{4 8 3 . 1 5}$ pressure $=\mathbf{3 9} .64)$

$\begin{array}{llll}19 & -2.622805000 & -0.000062000 & -0.000313000\end{array}$

$\begin{array}{llll}8 & -0.277992000 & -0.002545000 & -0.004808000\end{array}$

$\begin{array}{llll}6 & 1.101083000 & -0.000101000 & -0.000253000\end{array}$

$\begin{array}{llll}6 & 1.639583000 & 1.427575000 & 0.191390000\end{array}$

$\begin{array}{llll}6 & 1.639819000 & -0.877040000 & 1.142262000\end{array}$

$\begin{array}{llll}6 & 1.647365000 & -0.548234000 & -1.328937000\end{array}$

$\begin{array}{llll}1 & 1.281668000 & -1.901487000 & 1.022331000\end{array}$

$\begin{array}{llll}1 & 1.276568000 & 1.835867000 & 1.136745000\end{array}$

$1 \quad 1.288859000 \quad 0.065971000-2.157426000$

$\begin{array}{llll}1 & 2.739223000 & -0.562224000 & -1.361012000\end{array}$

$\begin{array}{llll}1 & 2.739223000 & -0.562224000 & -1.361012000 \\ 1 & 1.287503000 & -1.567416000 & -1.483923000\end{array}$

$\begin{array}{llll}1 & 2.731506000 & -0.898314000 & 1.176858000\end{array}$

$\begin{array}{llll}1 & 2.731506000 & -0.898314000 & 1.176858000 \\ 1 & 1.274598000 & -0.501608000 & 2.100308000\end{array}$

$\begin{array}{llll}1 & 2.731250000 & 1.467598000 & 0.198302000\end{array}$ 


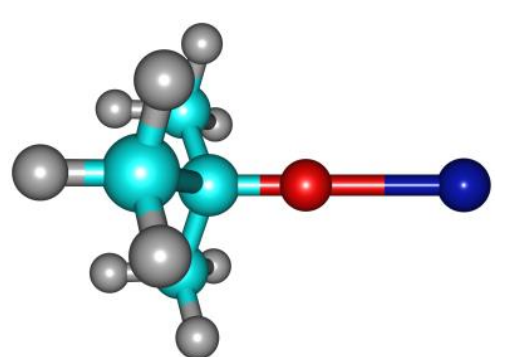

basis = def2-QZVP

$E=-833.029870142$ (gas)

$\mathbf{G}=\mathbf{- 8 3 2 . 9 6 8 2 9 2}$ (Temperature $=\mathbf{4 8 3 . 1 5}$, gas)

$\begin{array}{lrrr}19 & -2.535815000 & 0.000012000 & 0.000017000 \\ 8 & -0.305869000 & 0.000084000 & 0.000180000 \\ 6 & 1.065762000 & 0.000009000 & 0.000002000 \\ 6 & 1.598985000 & -1.157630000 & -0.859682000 \\ 6 & 1.599192000 & 1.323244000 & -0.572710000 \\ 6 & 1.599367000 & -0.165716000 & 1.432195000 \\ 1 & 1.229743000 & 2.153000000 & 0.031228000 \\ 1 & 1.229504000 & -1.049467000 & -1.880243000 \\ 1 & 1.229747000 & -1.103492000 & 1.848967000 \\ 1 & 2.690460000 & -0.170719000 & 1.475935000 \\ 1 & 1.229688000 & 0.651939000 & 2.052289000 \\ 1 & 2.690283000 & 1.363737000 & -0.590583000 \\ 1 & 1.229243000 & 1.451457000 & -1.590784000 \\ 1 & 2.690064000 & -1.193512000 & -0.885825000 \\ 1 & 1.228853000 & -2.103279000 & -0.461572000\end{array}$

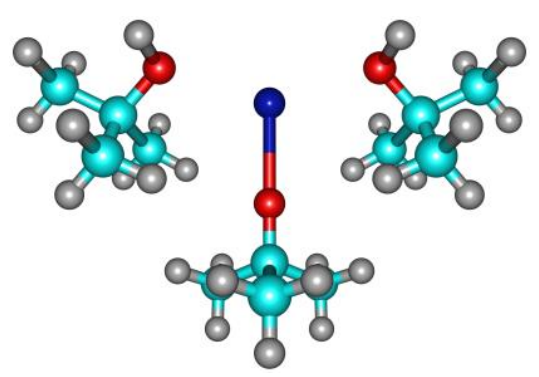

S1

basis = def2-SVP

$E=-1299.481077$

$E($ def2QZVP) $=-1300.45515$

$\mathbf{G}=\mathbf{- 1 2 9 9 . 1 9 1 5 2 9}$ (Temperature $=298.15$ pressure $=24.45$ )

$\begin{array}{lrrr}19 & 0.000467000 & -1.325019000 & -0.411203000 \\ 8 & -0.000006000 & 1.067815000 & -0.134280000 \\ 6 & 1.249037000 & 3.046003000 & -0.699080000 \\ 1 & 1.274648000 & 4.147384000 & -0.645283000 \\ 6 & -0.000480000 & 2.435830000 & -0.026404000 \\ 1 & 0.888183000 & 2.471842000 & 1.964833000 \\ 6 & -0.001953000 & 2.875134000 & 1.454134000 \\ 1 & -0.002342000 & 3.970911000 & 1.580114000 \\ 1 & -0.892818000 & 2.471359000 & 1.963183000 \\ 1 & -2.162455000 & 2.656702000 & -0.220642000 \\ 1 & -1.276810000 & 2.751310000 & -1.763453000 \\ 6 & -1.249183000 & 3.045396000 & -0.701153000 \\ 1 & -1.275282000 & 4.146761000 & -0.647351000 \\ 1 & 1.278452000 & 2.751865000 & -1.761313000 \\ 1 & 2.161699000 & 2.657674000 & -0.217110000 \\ 1 & -2.680997000 & -2.417512000 & 1.159307000 \\ 8 & -2.460749000 & -1.966406000 & 0.331485000 \\ 6 & -3.456820000 & -0.957566000 & 0.094302000 \\ 6 & -4.824104000 & -1.627494000 & 0.015723000 \\ 1 & -5.612307000 & -0.888519000 & -0.188578000 \\ 1 & -3.018717000 & -1.064158000 & -2.028667000 \\ 6 & -3.083013000 & -0.306787000 & -1.231639000 \\ 1 & -3.844363000 & 0.431665000 & -1.521848000 \\ 1 & -2.122589000 & 0.225408000 & -1.135280000 \\ 1 & -2.401813000 & 0.530487000 & 1.247550000\end{array}$

$\begin{array}{lr}1 & -3.599922000 \\ 6 & -3.400542000 \\ 1 & -4.149594000 \\ 1 & -5.064421000 \\ 1 & -4.833742000 \\ 1 & 2.682789000 \\ 8 & 2.461856000 \\ 6 & 3.457083000 \\ 6 & 3.399911000 \\ 1 & 4.148595000 \\ 1 & 2.122143000 \\ 6 & 3.083026000 \\ 1 & 3.843913000 \\ 1 & 3.019663000 \\ 1 & 4.835117000 \\ 1 & 5.065426000 \\ 6 & 4.824885000 \\ 1 & 5.612575000 \\ 1 & 3.599231000 \\ 1 & 2.400982000\end{array}$

$-0.411054000$

0.068376000

0.860072000

$-2.128301000$

$-2.381158000$

$-2.419318000$

$-1.967010000$

$-0.956961000$

0.067579000

0.859840000

0.226404000

$-0.304927000$

0.434455000

$-1.061402000$

$-2.378317000$

$-2.127644000$

$-1.625749000$

$-0.885935000$

$-0.412892000$

0.529240000

2.193645000

1.221982000

1.071611000

0.967192000

$-0.785791000$

1.157326000

0.330334000

0.094373000

1.223264000

1.074044000

$-1.134358000$

$-1.230884000$

$-1.519960000$

$-2.028852000$

$-0.787440000$

0.965941000

0.015100000

$-0.188128000$

2.194430000

1.249113000

\begin{tabular}{|c|c|c|c|}
\hline & $\begin{array}{l}=\text { def2-SVP } \\
1299.49178 \\
\text { 2QZVP) }=-130 \\
1299.204687(T\end{array}$ & $\begin{array}{l}.463636 \\
\text { mperature }=29\end{array}$ & \\
\hline 19 & 0.609178000 & -0.802163000 & -0.416812000 \\
\hline 8 & -1.819090000 & -1.048604000 & 0.095030000 \\
\hline 6 & -3.122642000 & -3.054572000 & 0.003831000 \\
\hline 1 & -4.140738000 & -3.475745000 & -0.015310000 \\
\hline 6 & -3.123474000 & -1.518901000 & 0.063077000 \\
\hline 1 & -3.333391000 & -1.301537000 & -2.091636000 \\
\hline 6 & -3.854635000 & -0.975626000 & -1.176900000 \\
\hline 1 & -4.900144000 & -1.319024000 & -1.234522000 \\
\hline 1 & -3.852024000 & 0.125820000 & -1.165155000 \\
\hline 1 & -3.933164000 & 0.020991000 & 1.376753000 \\
\hline 1 & -3.356534000 & -1.436864000 & 2.222333000 \\
\hline 6 & -3.886279000 & -1.078622000 & 1.324954000 \\
\hline 1 & -4.917668000 & -1.465978000 & 1.353230000 \\
\hline 1 & -2.597456000 & -3.462871000 & 0.882261000 \\
\hline 1 & -2.591501000 & -3.395745000 & -0.899265000 \\
\hline 1 & -1.590417000 & 0.261910000 & -0.412332000 \\
\hline 8 & -1.143834000 & 1.129988000 & -0.839970000 \\
\hline 6 & -1.294161000 & 2.252776000 & 0.002100000 \\
\hline 6 & -2.748674000 & 2.731603000 & -0.029845000 \\
\hline 1 & -2.891247000 & 3.644736000 & 0.568262000 \\
\hline 1 & -0.642748000 & 3.592320000 & -1.571088000 \\
\hline 6 & -0.376183000 & 3.349785000 & -0.530967000 \\
\hline 1 & -0.451703000 & 4.267283000 & 0.071733000 \\
\hline 1 & 0.671734000 & 3.010513000 & -0.516921000 \\
\hline 1 & 0.163939000 & 1.608308000 & 1.492923000 \\
\hline 1 & -1.501695000 & 1.030428000 & 1.782038000 \\
\hline 6 & -0.902348000 & 1.886932000 & 1.438371000 \\
\hline 1 & -1.060091000 & 2.728732000 & 2.129581000 \\
\hline 1 & -3.415286000 & 1.951948000 & 0.369897000 \\
\hline 1 & -3.050019000 & 2.942030000 & -1.067557000 \\
\hline 1 & 3.668676000 & -1.662555000 & -1.324742000 \\
\hline 8 & 3.194134000 & -1.105191000 & -0.690893000 \\
\hline 6 & 4.160439000 & -0.390786000 & 0.094313000 \\
\hline 6 & 5.024557000 & -1.393652000 & 0.851075000 \\
\hline 1 & 5.762984000 & -0.878453000 & 1.482190000 \\
\hline 1 & 2.728299000 & -0.154048000 & 1.715429000 \\
\hline 6 & 3.355260000 & 0.470546000 & 1.058489000 \\
\hline
\end{tabular}




$\begin{array}{rrrr}1 & 4.026106000 & 1.056199000 & 1.702174000 \\ 1 & 2.721231000 & 1.183568000 & 0.507059000 \\ 1 & 4.366845000 & 1.176607000 & -1.389517000 \\ 1 & 5.552429000 & -0.148910000 & -1.552500000 \\ 6 & 5.006261000 & 0.477712000 & -0.830181000 \\ 1 & 5.743225000 & 1.059014000 & -0.257460000 \\ 1 & 5.569466000 & -2.039884000 & 0.145251000 \\ 1 & 4.398116000 & -2.030425000 & 1.493084000\end{array}$

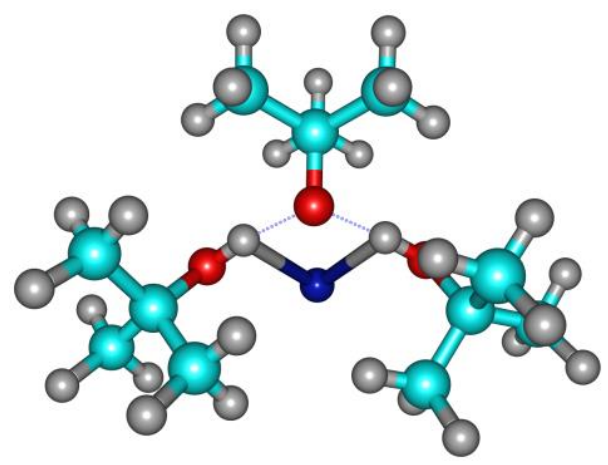

S3

basis = def2-SVP

$E=-1299.503745$

$E($ def2QZVP) $=-1300.473551$

$\mathbf{G}=\mathbf{- 1 2 9 9 . 2 0 9 4 9 6}($ Temperature $=\mathbf{2 9 8 . 1 5}$ pressure $=24.45)$

$\begin{array}{llll}1 & 1.311203000 & 0.254360000 & 0.094596000\end{array}$

$\begin{array}{llll}19 & -0.057533000 & -0.713204000 & 1.974142000\end{array}$

$\begin{array}{llll}6 & -2.810061000 & -0.995533000 & -0.409324000\end{array}$

$\begin{array}{llll}8 & -2.182585000 & -0.122912000 & 0.515957000\end{array}$

$\begin{array}{llll}6 & -2.908185000 & -0.308029000 & -1.772363000\end{array}$

$\begin{array}{llll}1 & -1.903220000 & -0.023723000 & -2.120619000\end{array}$

$\begin{array}{llll}1 & -3.364339000 & -0.971164000 & -2.522609000\end{array}$

$\begin{array}{llll}1 & -3.518547000 & 0.605042000 & -1.698322000\end{array}$

$\begin{array}{llll}6 & -4.199869000 & -1.307961000 & 0.133425000\end{array}$

$\begin{array}{llll}1 & -4.750723000 & -1.977998000 & -0.543167000\end{array}$

$\begin{array}{lll}-4.126242000 & -1.793943000 & 1.118654000\end{array}$

$\begin{array}{lll}-4.774878000 & -0.377266000 & 0.251323000\end{array}$

$\begin{array}{lll}-1.986872000 & -2.281291000 & -0.534162000\end{array}$

$\begin{array}{lll}-1.918770000 & -2.791620000 & 0.441450000\end{array}$

$\begin{array}{lll}-2.438242000 & -2.987692000 & -1.247232000\end{array}$

$\begin{array}{lll}-0.970023000 & -2.048556000 & -0.888672000\end{array}$

$\begin{array}{lll}-0.009987000 & 0.878624000 & -0.261656000\end{array}$

$\begin{array}{lll}-0.030199000 & 2.266038000 & -0.052903000\end{array}$

$\begin{array}{lll}1.227257000 & 2.896509000 & -0.660589000\end{array}$

$\begin{array}{lll}2.126643000 & 2.468655000 & -0.190662000\end{array}$

$\begin{array}{lll}1.266860000 & 2.680513000 & -1.739800000\end{array}$

$\begin{array}{lll}1.248820000 & 3.988975000 & -0.522982000\end{array}$

$\begin{array}{lll}-1.263728000 & 2.663517000 & -1.795387000\end{array}$

$\begin{array}{lll}-1.277287000 & 2.867245000 & -0.713023000\end{array}$

$\begin{array}{lll}-1.336953000 & 3.956952000 & -0.565128000\end{array}$

$\begin{array}{lll}-0.079417000 & 3.654249000 & 1.661406000\end{array}$

$\begin{array}{lll}-0.065232000 & 2.572709000 & 1.455050000\end{array}$

$\begin{array}{lll}0.829814000 & 2.156600000 & 1.949862000\end{array}$

$\begin{array}{lll}-0.972205000 & 2.140215000 & 1.912505000\end{array}$

$\begin{array}{lll}-2.182191000 & 2.405829000 & -0.288435000\end{array}$

$\begin{array}{lll}2.082513000 & -0.253384000 & 0.561953000\end{array}$

$\begin{array}{lll}2.861842000 & -0.962821000 & -0.386051000\end{array}$

$\begin{array}{lll}3.590514000 & 0.026791000 & -1.296853000\end{array}$

$\begin{array}{lll}2.863124000 & 0.634307000 & -1.856642000\end{array}$

$\begin{array}{lll}4.218595000 & 0.702871000 & -0.696605000\end{array}$

$\begin{array}{lll}4.233776000 & -0.494716000 & -2.021736000\end{array}$

$\begin{array}{lll}4.492580000 & -1.146093000 & 1.025903000\end{array}$

$\begin{array}{lll}3.867810000 & -1.798658000 & 0.397263000\end{array}$

$\begin{array}{lll}4.525010000 & -2.366240000 & -0.277912000\end{array}$

$\begin{array}{lll}2.515228000 & -2.415700000 & -1.986200000\end{array}$

$\begin{array}{lll}1.949153000 & -1.863227000 & -1.221071000\end{array}$

$\begin{array}{lll}1.185746000 & -1.252139000 & -1.727531000\end{array}$

$\begin{array}{lll}1.439054000 & -2.597209000 & -0.576373000\end{array}$

$\begin{array}{lll}3.345768000 & -2.512633000 & 1.053223000\end{array}$

$\begin{array}{lll}-1.342368000 & 0.306229000 & 0.081954000\end{array}$

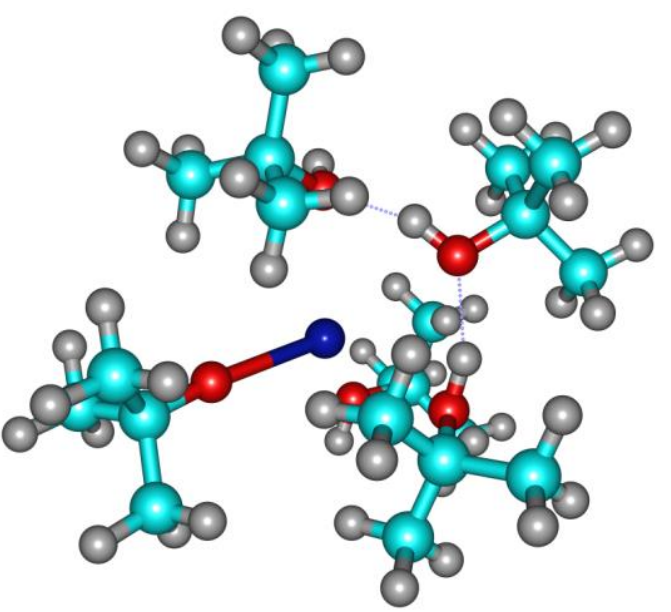

S4

$$
\begin{aligned}
& \text { basis = def2-SVP } \\
& E=-1767.85805 \\
& E(\text { def2QZVP })=-1300.473551
\end{aligned}
$$

$\mathbf{G}=\mathbf{- 1 7 6 5 . 7 8 3 6 8}($ Temperature $=\mathbf{2 9 8 . 1 5}$ pressure $=\mathbf{2 4 . 4 5}$ )

$19 \quad-0.296803000 \quad-0.465909000-0.765734000$

$\begin{array}{llll}8 & -2.664187000 & -0.836969000 & -0.400964000\end{array}$

$\begin{array}{llll}6 & -4.790745000 & -1.419784000 & -1.350458000\end{array}$

$1 \quad-5.845197000 \quad-1.664760000 \quad-1.138661000$

$6 \quad-3.964796000 \quad-1.124740000-0.079682000$

$\begin{array}{lll}-4.606206000 & 0.957876000 & 0.022216000\end{array}$

$\begin{array}{lll}-4.629868000 & 0.057920000 & 0.658826000\end{array}$

$\begin{array}{lll}-5.679787000 & -0.140144000 & 0.933698000\end{array}$

$\begin{array}{lll}-4.070535000 & 0.284109000 & 1.582277000\end{array}$

$-3.484871000 \quad-2.170354000 \quad 1.772980000$

$\begin{array}{lll}-3.574821000 & -3.226239000 & 0.340582000\end{array}$

$\begin{array}{lll}-4.042653000 & -2.362318000 & 0.841100000\end{array}$

$\begin{array}{lll}-5.075867000 & -2.639069000 & 1.111213000\end{array}$

$\begin{array}{lll}-4.341475000 & -2.267590000 & -1.893455000\end{array}$

$\begin{array}{lll}-4.768678000 & -0.543776000 & -2.019773000\end{array}$

$\begin{array}{lll}1.353483000 & -0.191066000 & 1.419318000\end{array}$

$\begin{array}{lll}1.109924000 & -1.133842000 & 1.478570000\end{array}$

$\begin{array}{lll}0.306863000 & -1.344454000 & 2.642527000\end{array}$

$\begin{array}{lll}1.167410000 & -1.134565000 & 3.885874000\end{array}$

$\begin{array}{lll}0.601088000 & -1.353775000 & 4.803145000\end{array}$

$\begin{array}{lll}0.670926000 & -3.477794000 & 2.527617000\end{array}$

$\begin{array}{lll}-0.182729000 & -2.783350000 & 2.552681000\end{array}$

$\begin{array}{lll}-0.814278000 & -3.036239000 & 3.416550000\end{array}$

$\begin{array}{lll}-0.776210000 & -2.925853000 & 1.636029000\end{array}$

$\begin{array}{lll}-1.503949000 & -0.541616000 & 1.739692000\end{array}$

$\begin{array}{lll}-0.508378000 & 0.667501000 & 2.618879000\end{array}$

$\begin{array}{lll}-0.872759000 & -0.372939000 & 2.628545000\end{array}$

$\begin{array}{lll}-1.499227000 & -0.503298000 & 3.524245000\end{array}$

$\begin{array}{lll}1.512869000 & -0.089743000 & 3.940838000\end{array}$

$\begin{array}{lll}2.049850000 & -1.791234000 & 3.852744000\end{array}$

$\begin{array}{lll}0.085454000 & 2.490010000 & -2.234159000\end{array}$

$\begin{array}{lll}-0.222481000 & 2.226972000 & -1.353510000\end{array}$

$\begin{array}{lll}-1.436604000 & 2.945506000 & -1.045141000\end{array}$

$\begin{array}{lll}-1.781345000 & 2.558998000 & 0.386359000\end{array}$

$\begin{array}{lll}-2.672235000 & 3.108722000 & 0.722915000\end{array}$

$\begin{array}{lll}-2.717693000 & 1.411611000 & -1.860867000\end{array}$

$\begin{array}{lll}-2.529685000 & 2.487180000 & -2.003205000\end{array}$

$\begin{array}{lll}-3.465720000 & 3.032231000 & -1.811050000\end{array}$

$\begin{array}{lll}-2.234421000 & 2.672520000 & -3.048093000\end{array}$

$\begin{array}{lll}-0.868503000 & 4.693624000 & -2.202078000\end{array}$

$\begin{array}{lll}-0.354062000 & 4.741824000 & -0.491278000\end{array}$

$\begin{array}{lll}-1.163898000 & 4.438643000 & -1.171985000\end{array}$

$\begin{array}{lll}-2.063202000 & 5.019501000 & -0.922282000\end{array}$

$\begin{array}{lll}-0.948199000 & 2.802334000 & 1.064137000\end{array}$

$\begin{array}{lll}-2.008954000 & 1.482106000 & 0.442221000\end{array}$

$\begin{array}{lll}0.743282000 & -3.409088000 & -1.861585000\end{array}$

$\begin{array}{lll}1.022458000 & -2.482681000 & -1.884268000\end{array}$

$\begin{array}{lll}2.451719000 & -2.437856000 & -1.964970000\end{array}$

$\begin{array}{lll}3.050662000 & -3.188289000 & -0.779316000\end{array}$

$\begin{array}{lll}4.149789000 & -3.164216000 & -0.815434000\end{array}$

$2.506494000 \quad-0.521512000 \quad-0.942618000$ 


$\begin{array}{lrr}2.818632000 & -0.960183000 & -1.903462000 \\ 3.906468000 & -0.829076000 & -1.995318000 \\ 2.337041000 & -0.407585000 & -2.726430000 \\ 2.429131000 & -2.522250000 & -4.130711000 \\ 2.589490000 & -4.113266000 & -3.337554000 \\ 2.893196000 & -3.055744000 & -3.287889000 \\ 3.986068000 & -3.008491000 & -3.401293000 \\ 2.732438000 & -4.242950000 & -0.791876000 \\ 2.717955000 & -2.732061000 & 0.164491000 \\ 0.984762000 & 1.931508000 & -0.035766000 \\ 1.571969000 & 1.422708000 & 0.558824000 \\ 2.788712000 & 2.150132000 & 0.762723000 \\ 2.510575000 & 3.315678000 & 1.708052000 \\ 3.430004000 & 3.879263000 & 1.924384000 \\ 2.553390000 & 3.320491000 & -1.050353000 \\ 3.296601000 & 2.653060000 & -0.585543000 \\ 4.233133000 & 3.216869000 & -0.466188000 \\ 3.482515000 & 1.809032000 & -1.266779000 \\ 3.951685000 & 0.318813000 & 0.712036000 \\ 3.382097000 & 0.782372000 & 2.339129000 \\ 3.773592000 & 1.170184000 & 1.386272000 \\ 4.735202000 & 1.664282000 & 1.585566000 \\ 2.097656000 & 2.941938000 & 2.657277000 \\ 1.780709000 & 4.008801000 & 1.261318000\end{array}$

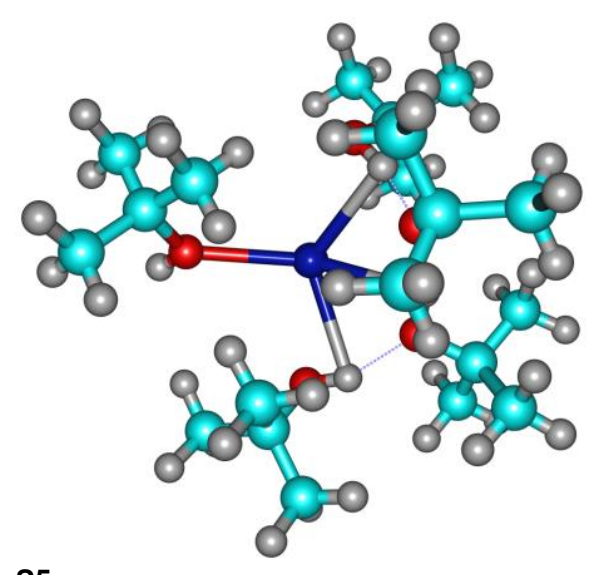

S5

basis = def2-SVP

$E=-1766.327514$

$E($ def2QZVP) $=-1767.880456$

$\mathbf{G}=\mathbf{- 1 7 6 5 . 8 1 0 1 1 6}$ (Temperature $=\mathbf{2 9 8 . 1 5}$ pressure $=24.45$ )

$19 \quad-0.618759000 \quad 0.148383000-0.808245000$

$\begin{array}{lllll}8 & 1.428886000 & 0.780411000 & 0.868408000\end{array}$

$\begin{array}{llll}6 & 0.959106000 & -0.319530000 & 2.950654000\end{array}$

$\begin{array}{llll}1 & 1.081558000 & -0.280886000 & 4.044652000\end{array}$

$\begin{array}{llll}6 & 1.583560000 & 0.896975000 & 2.256063000\end{array}$

$\begin{array}{llll}1 & -0.189782000 & 2.137001000 & 2.498852000\end{array}$

$\begin{array}{llll}6 & 0.883419000 & 2.170968000 & 2.745721000\end{array}$

$\begin{array}{llll}1 & 0.983657000 & 2.296383000 & 3.835207000\end{array}$

$\begin{array}{llll}1 & 1.314290000 & 3.057087000 & 2.254151000\end{array}$

$\begin{array}{llll}1 & 3.536779000 & 1.837410000 & 2.100165000\end{array}$

$\begin{array}{llll}1 & 3.596524000 & 0.066466000 & 2.286262000\end{array}$

$\begin{array}{llll}6 & 3.074069000 & 0.979267000 & 2.612740000\end{array}$

$\begin{array}{llll}1 & 3.235352000 & 1.096215000 & 3.696064000\end{array}$

$\begin{array}{llll}1 & 1.426016000 & -1.247651000 & 2.583886000\end{array}$

$\begin{array}{llll}1 & -0.119067000 & -0.367205000 & 2.726178000\end{array}$

$\begin{array}{llll}1 & 0.811324000 & 1.997186000 & 0.166716000\end{array}$

$\begin{array}{llll}8 & 0.280389000 & 2.670642000 & -0.391177000\end{array}$

$\begin{array}{llll}6 & 1.146407000 & 3.218132000 & -1.372043000\end{array}$

$\begin{array}{lllll}6 & 2.371343000 & 3.822143000 & -0.683022000\end{array}$

$\begin{array}{llll}1 & 3.066490000 & 4.266724000 & -1.410853000\end{array}$

$\begin{array}{llll}1 & 0.030267000 & 5.070750000 & -1.407219000\end{array}$

$\begin{array}{llll}6 & 0.360758000 & 4.293181000 & -2.112271000\end{array}$

$\begin{array}{llll}1 & 0.972123000 & 4.765368000 & -2.895369000\end{array}$

$1 \quad-0.532452000 \quad 3.855165000 \quad-2.584283000$

$\begin{array}{llll}1 & 0.720996000 & 1.720060000 & -2.900979000\end{array}$

$\begin{array}{llll}1 & 2.052094000 & 1.288860000 & -1.772126000\end{array}$

$\begin{array}{llll}6 & 1.584843000 & 2.111449000 & -2.337112000\end{array}$

$\begin{array}{llll}1 & 2.316842000 & 2.477539000 & -3.072519000\end{array}$

2.906498000

2.058807000

$-3.908628000$

$-3.231582000$

$-3.865851000$

$-4.542337000$

$-5.017429000$

$-2.009918000$

$-2.746077000$

$-3.151638000$

$-2.236661000$

$-4.379271000$

$-5.651345000$

$-4.877777000$

$-5.375924000$

$-5.320675000$

$-3.803715000$

$-0.699918000$

0.183409000

$-1.454472000$

$-0.760747000$

$-1.339105000$

$-2.745512000$

$-2.832157000$

$-3.474003000$

$-3.315341000$

$-2.109543000$

$-0.554162000$

$-1.559749000$

$-2.098401000$

0.237146000

$-0.639537000$

1.585522000

1.638582000

2.850839000

3.865639000

4.873806000

2.304574000

2.675399000

3.628546000

1.947140000

2.546393000

3.459709000

3.309239000

4.255957000

3.900621000

3.583919000

\subsection{0}

4.604558000

$-0.002839000$

0.314853000

1.106791000

2.305887000

2.949006000

2.178221000

1.558672000

2.160700000

0.687092000

$-0.626807000$

$-0.126109000$

0.241494000

0.815491000

1.972024000

2.904559000

$-2.521812000$

$-2.333152000$

$-3.306572000$

$-4.652694000$

$-5.306270000$

$-3.974170000$

$-3.487634000$

$-4.106613000$

$-2.507779000$

$-1.614667000$

$-2.341032000$

$-2.560634000$

$-3.155064000$

$-4.506044000$

$-5.160707000$

$-1.367032000$

$-0.488548000$

$-1.965977000$

$-0.889891000$

$-1.316742000$

$-2.448147000$

$-2.962697000$

$-3.453461000$

$-3.741909000$

$-3.424544000$

$-1.979911000$

$-2.694559000$

$-3.230738000$

$-0.105838000$

$-0.420752000$
$-0.122839000$

0.025318000

$-1.326155000$

$-0.711367000$

0.304326000

$-0.350829000$

0.404039000

0.696809000

1.233999000

2.059314000

1.677625000

1.504561000

0.355134000

1.047794000

1.842440000

$-1.054916000$

$-0.904511000$

$-1.229883000$

$-0.840118000$

$-0.308447000$

$-0.112118000$

0.557463000

$-1.913008000$

$-0.930005000$

$-0.286719000$

$-1.064560000$

0.886266000

1.413483000

1.021876000

1.774755000

0.331159000

$-1.080654000$

$-0.337640000$

0.261285000

$-0.569611000$

$-0.956780000$

$-1.066971000$

$-2.612493000$

$-1.712558000$

$-1.959230000$

$-1.438244000$

1.008078000

1.518619000

0.695227000

0.529816000

$-0.184625000$

$-1.911606000$

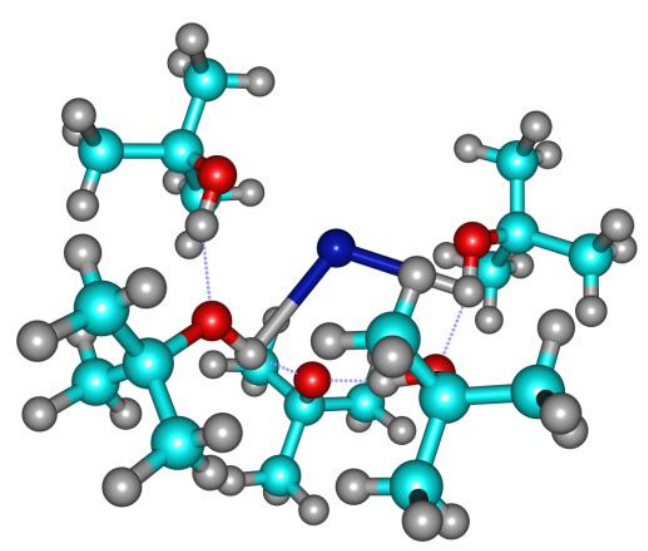

S6

basis = def2-SVP

$E=-1766.330705$

$E($ def2QZVP) $=-1767.882517$

$\mathbf{G}=\mathbf{- 1 7 6 5 . 8 0 6 8 9 7}($ Temperature $=\mathbf{2 9 8 . 1 5}$ pressure $=\mathbf{2 4 . 4 5}$ )

$\begin{array}{llll}1 & -1.578812000 & -0.259896000 & 2.811555000\end{array}$

$\begin{array}{llll}6 & -1.305761000 & -1.317881000 & 2.957741000\end{array}$

$\begin{array}{llll}6 & 0.068144000 & -1.596586000 & 2.337864000\end{array}$

$\begin{array}{llll}8 & 0.018004000 & -1.325719000 & 0.962850000\end{array}$

$\begin{array}{llll}6 & 1.116261000 & -0.692639000 & 2.998349000\end{array}$ 


\subsection{0}

1.209315000

2.101994000

0.443968000

1.443548000

$-0.280144000$

0.457298000

$-1.318892000$

$-2.075352000$

2.844751000

2.716957000

3.031614000

2.192644000

2.715055000

1.653094000

3.324951000

2.919547000

4.493684000

4.702167000

4.713818000

5.170094000

1.681569000

3.383717000

2.368838000

1.713430000

2.231423000

2.940768000

1.999136000

3.999593000

3.537583000

4.780667000

4.478814000

4.081197000

3.581491000

2.812525000

4.329108000

2.953940000

1.488115000

1.251516000

$-5.176249000$

$-6.062127000$

$-2.770625000$

$-3.887766000$

$-3.816873000$

$-4.660717000$

$-2.880742000$

$-3.846041000$

$-3.785408000$

$-4.635313000$

$-2.857669000$

$-3.779300000$

$-5.203248000$

$-5.233850000$

$-2.751596000$

$-0.183724000$

$-2.204124000$

$-1.310166000$

$-2.097050000$

$-1.710310000$

$-1.636189000$

$-0.738423000$

$-2.463384000$

$-1.033824000$

$-0.065672000$

$-0.889721000$

$-1.340180000$

$-3.460704000$

$-3.452244000$

$-4.228124000$

$-3.735870000$
0.368529000

$-0.889410000$

$-0.850956000$

$-3.065707000$

$-3.271721000$

$-3.721401000$

$-3.327878000$

$-1.528363000$

$-1.938244000$

1.483677000

2.549665000

2.789439000

1.959702000

4.224705000

4.448738000

4.933310000

4.377086000

2.457025000

2.570824000

1.417294000

3.118484000

2.848042000

3.133781000

1.015514000

$-3.329608000$

$-2.970907000$

$-1.646967000$

$-0.675836000$

$-1.844911000$

$-2.242760000$

$-2.549383000$

$-0.883726000$

$-0.144676000$

$-1.105676000$

$-0.940262000$

$-1.807877000$

$-3.740157000$

$-2.852271000$

$-1.073628000$

1.324902000

1.762255000

1.337529000

1.908433000

3.409379000

3.925769000

3.821330000

3.618718000

1.593674000

2.016837000

2.013774000

0.503671000

0.234706000

1.525198000

0.361034000

1.082827000

$-1.243765000$

$-1.357938000$

$-1.918102000$

$-3.373687000$

$-3.940808000$

$-3.417048000$

$-3.854679000$

$-1.228394000$

$-1.220401000$

$-1.750647000$

$-0.193721000$

$-1.828156000$

$-2.332521000$

$-2.299862000$

$-0.773382000$
2.871415000

4.077926000

2.532998000

2.567716000

2.152624000

2.058645000

3.637265000

4.038416000

2.472360000

1.562087000

1.315956000

$-0.160995000$

$-0.963384000$

$-0.556698000$

$-0.371736000$

0.021687000

$-1.626840000$

$-0.450560000$

$-1.524869000$

$-0.160417000$

0.110469000

1.551028000

1.967239000

$-0.749945000$

$-0.193256000$

$-1.095804000$

$-0.812044000$

$-0.375171000$

0.274067000

1.191174000

$-0.049486000$

0.517377000

$-1.889223000$

$-2.086776000$

$-2.857208000$

$-2.484173000$

$-1.407064000$

$-1.898478000$

0.245854000

$-0.480748000$

0.003214000

$-0.580737000$

0.096521000

$-0.148515000$

0.331109000

0.259218000

$-1.228314000$

1.588397000

2.144246000

2.008919000

1.745845000

$-0.326207000$

$-1.561337000$

$-0.416760000$

$-0.122156000$

$-0.201576000$

0.319212000

$-1.452130000$

$-1.195624000$

$-2.135595000$

$-0.681819000$

$-0.552809000$

$-2.312983000$

$-1.786443000$

$-3.270875000$

$-2.542898000$

$-2.125949000$

$-3.103355000$

$-1.493527000$

$-2.280829000$

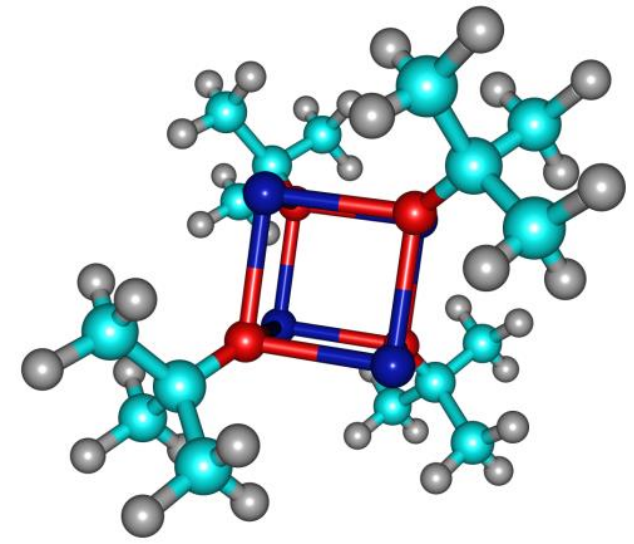

S7

$$
\begin{aligned}
& \text { basis }=\text { def2-SVP } \\
& E=-3330.829761 \\
& E(\text { def2QZVP })=-3332.351039
\end{aligned}
$$

$\mathbf{G}=\mathbf{- 3 3 3 0 . 4 8 5 5 4 6}($ Temperature $=\mathbf{2 9 8 . 1 5}$ pressure $=\mathbf{2 4 . 4 5})$

$-1.284820000$

$-1.546764000$

$-2.237343000$

$-1.364952000$

1.913473000

1.178897000

$19-1.637146000$

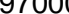

1.152911000

1.002465000

2.415796000

2.928304000

1.617835000

$-0.125857000$

0.575561000

1.026991000

$-0.001275000$

2.047574000

3.365484000

2.595380000

3.111750000

3.183023000

1.736754000

$-1.456200000$

$-0.966422000$

$-2.095866000$

$-0.676308000$

$-2.924036000$

$-2.161246000$

$-3.609588000$

$-3.508449000$

$-3.335760000$

$-4.035831000$

$-2.873120000$

$-3.923467000$

1.387267000

$-1.859660000$

$-1.159097000$

$-1.013097000$

1.534945000

1.825688000

2.554084000

2.486198000

2.648620000

3.313568000

4.092088000

3.812498000

3.959407000

2.890932000

3.453898000

4.233889000

1.244213000

1.137755000

$-2.938198000$

$-2.463061000$

$-3.526294000$

$-3.640481000$

$-2.560093000$

0.976920000

$-1.569177000$

$-1.834358000$

$-2.555076000$

$-2.510133000$

$-2.868109000$

$-3.452253000$

$-4.226492000$

$-3.964252000$

$-3.873772000$

$-2.717629000$ 


$\begin{array}{lrr}2.930018000 & -3.257625000 & 0.076918000 \\ 4.376300000 & -3.135532000 & -0.956606000 \\ 3.978346000 & -1.808724000 & 0.164294000 \\ 3.565879000 & -0.915426000 & -2.401500000 \\ 3.998880000 & -0.142024000 & -1.743724000 \\ 4.399122000 & -1.436430000 & -2.901016000 \\ 2.967154000 & -0.414357000 & -3.181724000\end{array}$

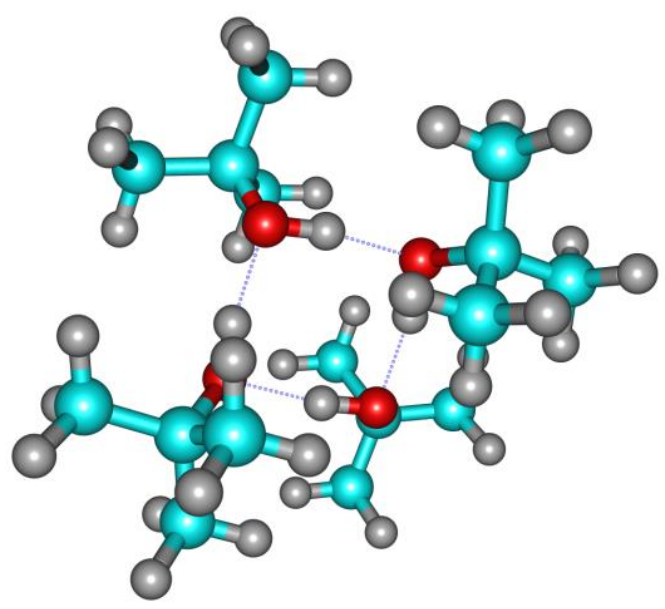

basis = def2-SVP

$E=-933.608532$

$E($ def2QZVP) $=-934.7851394$

$\mathbf{G}=\mathbf{- 9 3 3 . 1 8 6 2 1 2}($ Temperature $=\mathbf{2 9 8 . 1 5}$ pressure $=24.45)$

$\begin{array}{lll}-1.937030000 & 1.961699000 & -1.111695000\end{array}$

$\begin{array}{lll}-1.366819000 & 1.341608000 & 0.046101000\end{array}$

$\begin{array}{lll}-1.215201000 & 1.459013000 & -2.359545000\end{array}$

$\begin{array}{lll}-0.148639000 & 1.728376000 & -2.316614000\end{array}$

$\begin{array}{lll}-1.298470000 & 0.363989000 & -2.432692000\end{array}$

$\begin{array}{lll}-1.647181000 & 1.902264000 & -3.268960000\end{array}$

$\begin{array}{lll}-1.795650000 & 3.475659000 & -0.981475000\end{array}$

$\begin{array}{lll}-0.732140000 & 3.759042000 & -0.955659000\end{array}$

$\begin{array}{lll}-2.267090000 & 3.991027000 & -1.831288000\end{array}$

$\begin{array}{lll}-2.271925000 & 3.822636000 & -0.052188000\end{array}$

$\begin{array}{lll}-3.401862000 & 1.548715000 & -1.133950000\end{array}$

$\begin{array}{lll}-3.487278000 & 0.455379000 & -1.228781000\end{array}$

$\begin{array}{lll}-3.900199000 & 1.860275000 & -0.203434000\end{array}$

$\begin{array}{lll}-3.924864000 & 2.012714000 & -1.982407000\end{array}$

$\begin{array}{lll}1.368561000 & -1.341304000 & 0.046777000\end{array}$

$\begin{array}{lll}0.387612000 & -1.455716000 & 0.020255000\end{array}$

$\begin{array}{lll}3.921220000 & -2.009247000 & -1.990078000\end{array}$

$\begin{array}{lll}3.400182000 & -1.544908000 & -1.140615000\end{array}$

$\begin{array}{lll}3.483105000 & -0.451551000 & -1.237903000\end{array}$

$\begin{array}{lll}3.902123000 & -1.854170000 & -0.211257000\end{array}$

$\begin{array}{lll}0.735285000 & -3.760618000 & -0.955185000\end{array}$

$\begin{array}{lll}1.936220000 & -1.960547000 & -1.112905000\end{array}$

$\begin{array}{lll}1.291075000 & -0.364252000 & -2.432182000\end{array}$

$\begin{array}{lll}1.209544000 & -1.459390000 & -2.358610000\end{array}$

$\begin{array}{lll}0.143606000 & -1.730136000 & -2.311750000\end{array}$

$\begin{array}{lll}2.270394000 & -3.989021000 & -1.831626000\end{array}$

$\begin{array}{lll}1.638905000 & -1.902163000 & -3.269462000\end{array}$

$\begin{array}{lll}2.275580000 & -3.820091000 & -0.052428000\end{array}$

$\begin{array}{lll}1.798089000 & -3.474588000 & -0.981688000\end{array}$

$\begin{array}{lll}-1.340620000 & -1.368017000 & -0.046020000\end{array}$

$\begin{array}{lll}-1.961357000 & -1.936673000 & 1.112155000\end{array}$

$\begin{array}{lll}-1.546498000 & -3.400886000 & 1.138358000\end{array}$

$\begin{array}{lll}-2.010989000 & -3.922488000 & 1.987370000\end{array}$

$\begin{array}{lll}-1.856239000 & -3.901830000 & 0.208619000\end{array}$

$\begin{array}{lll}-0.453178000 & -3.484519000 & 1.234764000\end{array}$

$\begin{array}{lll}-1.903280000 & -1.642434000 & 3.269110000\end{array}$

$\begin{array}{lll}-1.461102000 & -1.211040000 & 2.358893000\end{array}$

$\begin{array}{lll}-1.733509000 & -0.145418000 & 2.313554000\end{array}$

$\begin{array}{lll}-3.820392000 & -2.274958000 & 0.050119000\end{array}$

$\begin{array}{lll}-3.475264000 & -1.797800000 & 0.979696000\end{array}$

$\begin{array}{lll}-3.760443000 & -0.734771000 & 0.953103000\end{array}$

$\begin{array}{lll}-3.990734000 & -2.269762000 & 1.829162000\end{array}$

$\begin{array}{lll}-0.365768000 & -1.290885000 & 2.432330000\end{array}$ $\begin{array}{llll}8 & 1.342352000 & 1.365751000 & -0.045879000\end{array}$

$\begin{array}{lll}1.962139000 & 1.935438000 & 1.112347000\end{array}$

$\begin{array}{lll}1.456545000 & 1.214903000 & 2.359859000\end{array}$

$\begin{array}{lll}1.725849000 & 0.148244000 & 2.317838000\end{array}$

$\begin{array}{lll}1.898094000 & 1.647249000 & 3.269925000\end{array}$

$\begin{array}{lll}0.361360000 & 1.298369000 & 2.430858000\end{array}$

$\begin{array}{lll}3.825358000 & 2.264922000 & 0.055414000\end{array}$

$\begin{array}{lll}3.475994000 & 1.790873000 & 0.984981000\end{array}$

$\begin{array}{lll}3.757481000 & 0.726824000 & 0.961227000\end{array}$

$\begin{array}{lll}2.015798000 & 3.923440000 & 1.982217000\end{array}$

$\begin{array}{lll}1.552106000 & 3.401084000 & 1.133210000\end{array}$

$\begin{array}{lll}0.458793000 & 3.488845000 & 1.225826000\end{array}$

$\begin{array}{lll}1.866310000 & 3.898492000 & 0.203043000\end{array}$

$\begin{array}{lll}3.990702000 & 2.262968000 & 1.834873000\end{array}$

$\begin{array}{lll}1.456829000 & 0.384873000 & -0.017178000\end{array}$

$\begin{array}{lll}-0.386055000 & 1.457688000 & 0.018706000\end{array}$

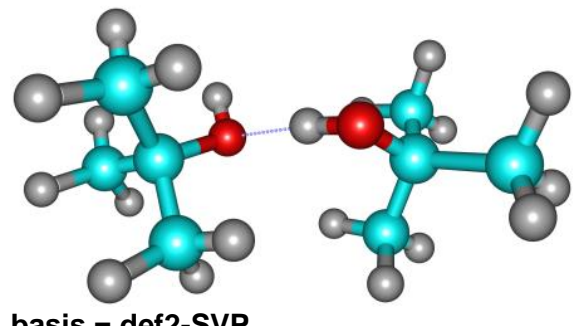

basis $=$ def2-SVP

$E=-466.789425$

$E($ def2QZVP) $=-467.3821373$

$\mathbf{G}=\mathbf{- 4 6 6 . 5 9 1 1 1 9}($ Temperature $=298.15$ pressure $=24.45)$

$\begin{array}{llll}1 & -1.417739000 & 1.486769000 & -0.982941000\end{array}$

$\begin{array}{llll}8 & -1.157256000 & 0.580678000 & -0.760299000\end{array}$

$\begin{array}{llll}6 & -2.206101000 & -0.010338000 & 0.023985000\end{array}$

$\begin{array}{llll}6 & -2.383018000 & 0.798064000 & 1.306019000\end{array}$

$\begin{array}{llll}-3.171255000 & 0.364981000 & 1.938843000\end{array}$

$\begin{array}{llll}1 & -0.779577000 & -1.387275000 & 0.879022000\end{array}$

$\begin{array}{llll}6 & -1.735467000 & -1.423917000 & 0.336623000\end{array}$

$\begin{array}{lll}-2.476296000 & -1.946471000 & 0.958116000\end{array}$

$\begin{array}{lll}-1.591934000 & -1.996124000 & -0.592277000\end{array}$

$\begin{array}{lll}-3.328787000 & -0.572740000 & -1.740217000\end{array}$

$\begin{array}{lll}-3.802124000 & 1.002472000 & -1.044363000\end{array}$

$\begin{array}{lll}-3.488488000 & -0.024819000 & -0.799946000\end{array}$

$\begin{array}{lll}-4.305792000 & -0.508668000 & -0.245714000\end{array}$

$\begin{array}{lll}-2.665437000 & 1.836433000 & 1.070474000\end{array}$

$\begin{array}{lll}-1.443683000 & 0.813901000 & 1.879366000\end{array}$

$\begin{array}{lll}0.342951000 & 0.506669000 & 0.271622000\end{array}$

$\begin{array}{lll}1.118219000 & 0.348427000 & 0.844334000\end{array}$

$\begin{array}{lll}2.207537000 & 0.007959000 & -0.005729000\end{array}$

$\begin{array}{lll}1.850895000 & -1.229752000 & -0.829552000\end{array}$

$\begin{array}{lll}2.674782000 & -1.511097000 & -1.502590000\end{array}$

$\begin{array}{lll}3.145111000 & -1.104566000 & 1.594803000\end{array}$

$\begin{array}{lll}3.388750000 & -0.277037000 & 0.911632000\end{array}$

$4.282359000 \quad-0.551220000 \quad 0.332613000$

$\begin{array}{lll}3.621327000 & 0.611944000 & 1.517139000\end{array}$

$\begin{array}{lll}2.730355000 & 2.086991000 & -0.332686000\end{array}$

$\begin{array}{lll}1.644836000 & 1.401673000 & -1.572675000\end{array}$

$\begin{array}{lll}2.513047000 & 1.187945000 & -0.928838000\end{array}$

$\begin{array}{lll}3.377026000 & 0.979523000 & -1.577422000\end{array}$

$\begin{array}{lll}0.956732000 & -1.031981000 & -1.441032000\end{array}$

$1.636515000-2.082684000-0.167516000$ 


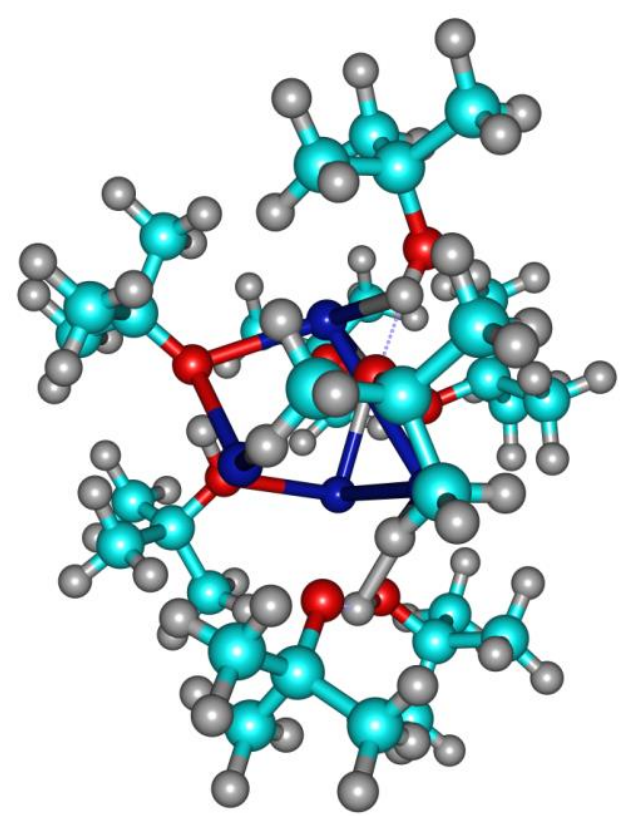

S8

\section{basis $=$ def2-SVP \\ $E=-4264.492834$ \\ $E($ def2QZVP) $=-4267.170424$}

$\mathbf{G}=\mathbf{- 4 2 6 3 . 6 6 6 6 1 6}($ Temperature $=\mathbf{2 9 8 . 1 5}$ pressure $=24.45)$

$8-3.104987000$

$6-4.180716000-2.864945000$

$6 \quad-4.355075000 \quad-2.003973000$

$\begin{array}{lll}1 & -6.242435000 & -1.058476000\end{array}$

$6-5.207427000$

$1-5.229623000$

$1-4.812552000$

$-4.812552000$

$-0.774243000$

$-0.085403000$

$-0.230548000$

$-3.165135000$

$-5.183937000 \quad-2.172087000$

$-5.050657000$

$-2.804802000$

$-3.673133000$

$-3.824504000$

$-2.350804000$

$-2.023647000$

$-0.104784000$

0.638388000

0.522138000

$-0.291408000$

1.041895000

0.007368000

$-0.106430000$

$-0.683041000$

$-1.979187000$

$-1.967648000$

$-1.751523000$

$-2.432384000$

0.424194000

1.688146000

0.714471000

1.490917000

2.916576000

3.771656000

1.601144000

0.424485000

0.434622000

0.516358000

$-0.527562000$

0.582187000

2.342742000

1.567013000

1.739636000

2.941290000

3.051273000

2.533803000
1.547994000

$-0.184130000$

$-0.650884000$

1.068725000

0.493883000

0.735463000

1.593814000

$-0.138768000$

1.849539000

3.063667000

2.172560000

2.459675000

0.053759000

$-0.926688000$

1.169172000

$-2.512603000$

$-4.729539000$

$-5.543035000$

$-3.545195000$

$-2.674118000$

$-3.052365000$

$-3.847519000$

$-2.238921000$

$-4.407865000$

$-4.830715000$

$-4.026549000$

$-3.184908000$

$-5.140595000$

$-4.395295000$

$-2.414433000$

1.827210000

3.754701000

3.144935000

3.112286000

4.113635000

4.007052000

5.021185000

3.591239000

2.687801000

2.362800000

3.050017000

4.036732000

3.800648000

4.775473000

0.870643000
3.088147000

3.705246000

4.346517000

3.984583000

6.135633000

5.432786000

5.680486000

5.582396000

2.757195000

4.494650000

3.746730000

3.808399000

2.656509000

3.898209000

3.154501000

0.959428000

3.846308000

2.822900000

19

1.258168000

$-1.497817000$

$-2.272370000$

$-2.479760000$

$-1.801031000$

$-0.133830000$

$-0.548237000$

0.218375000

$-0.778665000$

$-3.789038000$

$-3.218067000$

$-2.909779000$

$-2.572005000$

$-3.191917000$

$-1.502398000$

4.907783000

5.753582000

4.467267000

5.300801000

3.278280000

2.501473000

2.848526000

4.059825000

4.523771000

4.987944000

3.779422000

5.309717000

$19-2.091257000$

$-0.569147000$

$-0.900742000$

$19 \quad 0.513333000$

0.857898000

1.389845000

0.451650000

1.830919000

1.059169000

2.023952000

2.706414000

2.466800000

3.974799000

3.369905000

3.143189000

3.090668000

2.154498000

1.222337000

0.857680000

1.202814000

1.568887000

0.896594000

$-0.638859000$

$-1.605586000$

0.165499000

$-0.528448000$

$-1.524453000$

$-1.189171000$

$-1.572142000$
0.759015000

2.467070000

2.751789000

1.034687000

1.255180000

0.924952000

$-0.114395000$

1.551940000

0.245365000

0.209074000

0.070410000

$-0.975496000$

2.567247000

3.176159000

$-0.218345000$

$-0.760146000$

$-2.265697000$

$-1.407146000$

2.452437000

2.905079000

4.548464000

5.616535000

4.288385000

4.866589000

5.120103000

4.959615000

6.195486000

4.008997000

5.699206000

4.650522000

4.519500000

3.980181000

4.227731000

$-1.505324000$

$-2.153490000$

$-1.101103000$

$-0.658803000$

$-3.383909000$

$-3.954928000$

$-2.965692000$

$-4.095762000$

$-2.916718000$

$-2.145434000$

$-3.454452000$

$-3.630503000$

0.312767000

$-3.711948000$

$-2.387152000$

$-1.739868000$

1.112785000

2.404741000

2.136965000

1.290978000

$-0.387176000$

$-0.020990000$

0.109363000

$-0.782266000$

1.674391000

0.969940000

1.679346000

2.699136000

2.599267000

3.335343000

$-3.794929000$

$-4.836722000$

$-3.258887000$

$-3.355075000$

$-4.600188000$

$-4.457588000$

$-4.359408000$

$-5.668840000$

$-4.292703000$

$-5.285221000$

$-3.624337000$

0.944744000

2.457594000

3.304628000

2.001507000

2.300893000

1.521217000

1.261089000

0.628582000

3.624273000

3.965105000

3.170251000

2.828748000

2.784263000

1.635557000

0.471921000

1.697475000

$-0.574912000$

$-0.166511000$

0.171087000

$-0.037377000$

$-1.533183000$

$-1.693818000$

$-0.105733000$

1.179250000

0.188672000

$-0.587731000$

0.195796000

0.718048000

0.756776000

0.882534000

1.921383000

$-1.739596000$

$-2.250997000$

$-1.386842000$

$-1.666749000$

$-2.311287000$ 


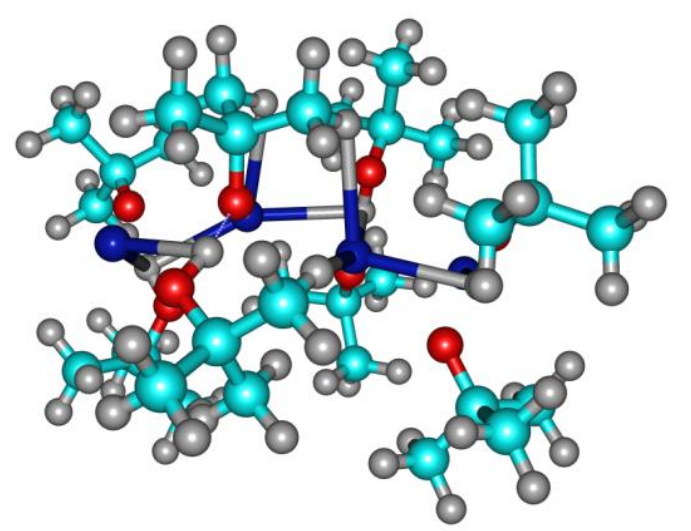

s9

basis $=$ def2-SVP
$E=-4264.490015$

\section{$E($ def2QZVP $)=-4267.16944$}

$\mathbf{G}=\mathbf{- 4 2 6 3 . 6 7 6 8 7}($ Temperature $=298.15$ pressure $=24.45)$

\begin{tabular}{|c|c|c|c|c|}
\hline & & & & \\
\hline 3.323675000 & 0.793748000 & 1.264812000 & 1 & -0.801162000 \\
\hline 5.063413000 & 0.344548000 & 2.866161000 & 8 & -3.091771000 \\
\hline 5.757588000 & 0.790512000 & 3.595769000 & 6 & -4.360058000 \\
\hline 4.109135000 & 1.384562000 & 2.249467000 & 1 & -5.148873000 \\
\hline 2.589987000 & 1.143657000 & 3.784425000 & 6 & -3.662174000 \\
\hline 3.209019000 & 1.947866000 & 3.357311000 & 1 & -1.743259000 \\
\hline 3.790031000 & 2.409102000 & 4.171814000 & 6 & -2.518714000 \\
\hline 2.531613000 & 2.709998000 & 2.940713000 & 1 & -2.875607000 \\
\hline 5.633744000 & 2.148854000 & 0.890438000 & 1 & -2.058799000 \\
\hline 5.537645000 & 3.054674000 & 2.422663000 & 1 & -4.194115000 \\
\hline 4.941990000 & 2.532240000 & 1.657261000 & 1 & -5.519767000 \\
\hline 4.274042000 & 3.266287000 & 1.178078000 & 6 & -4.671511000 \\
\hline 5.666580000 & -0.135225000 & 2.076591000 & $\begin{array}{l}1 \\
1\end{array}$ & -5.064833000 \\
\hline 4.492332000 & -0.434233000 & 3.402104000 & 1 & -3.632746000 \\
\hline 3.899648000 & 0.162260000 & 0.137184000 & 1 & -4.838326000 \\
\hline 0.847303000 & -2.300370000 & -0.798176000 & 1 & -3.787220000 \\
\hline 1.913794000 & -4.446770000 & -0.539107000 & 19 & -3.236707000 \\
\hline 1.717025000 & -4.461259000 & 0.545461000 & 8 & -4.291322000 \\
\hline 0.841156000 & -3.636066000 & -1.272880000 & 6 & -5.930646000 \\
\hline 2.134557000 & -3.113539000 & -2.927062000 & 1 & -6.625861000 \\
\hline 1.158899000 & -3.595695000 & -2.763541000 & 6 & -5.152502000 \\
\hline 1.196182000 & -4.611582000 & -3.183951000 & $\begin{array}{l}1 \\
6\end{array}$ & -5.611259000 \\
\hline 0.393737000 & -3.022689000 & -3.308135000 & 6 & -6.150550000 \\
\hline-1.307544000 & -3.695063000 & -1.602357000 & 1 & -6.718503000 \\
\hline-0.798673000 & -4.216332000 & 0.036308000 & 1 & -6.867686000 \\
\hline-0.541542000 & -4.249151000 & -1.035638000 & 1 & -3.629391000 \\
\hline-0.580902000 & -5.299356000 & -1.360975000 & 1 & -5.014989000 \\
\hline 2.910586000 & -4.015996000 & -0.731290000 & 6 & -4.362651000 \\
\hline 1.933339000 & -5.491375000 & -0.883034000 & $\begin{array}{l}1 \\
1\end{array}$ & -3.830191000 \\
\hline 0.609806000 & -2.239437000 & 0.216554000 & $\begin{array}{l}1 \\
1\end{array}$ & -5.228991000 \\
\hline 3.054637000 & -1.895359000 & 1.337223000 & 1 & -6.512737000 \\
\hline 0.420641000 & -1.605396000 & 1.510728000 & 19 & -1.720903000 \\
\hline 0.505259000 & -1.160451000 & 3.857216000 & 8 & -1.021147000 \\
\hline 0.151480000 & -1.463784000 & 4.855177000 & 6 & -0.208155000 \\
\hline-0.012106000 & -2.086337000 & 2.747317000 & 1 & -0.372011000 \\
\hline 0.152790000 & -4.193513000 & 2.211945000 & 6 & 0.094783000 \\
\hline 0.503248000 & -3.512970000 & 3.003838000 & $\begin{array}{l}1 \\
6\end{array}$ & 1.209936000 \\
\hline 1.606395000 & -3.554247000 & 3.029880000 & 1 & 1.047350000 \\
\hline 0.150010000 & -3.906076000 & 3.969844000 & 1 & 1.554611000 \\
\hline-1.965088000 & -1.125711000 & 2.604411000 & 1 & \\
\hline-1.912211000 & -2.443541000 & 3.802923000 & 1 & 1.180273000 \\
\hline-1.549303000 & -2.123692000 & 2.813621000 & 1 & 0.431201000 \\
\hline-1.948981000 & -2.842075000 & 2.075385000 & 6 & 0.298213000 \\
\hline 0.173433000 & -0.128399000 & 3.664584000 & 1 & -0.584692000 \\
\hline 1.608658000 & -1.159497000 & 3.879180000 & 1 & 0.620028000 \\
\hline 1.335103000 & 0.315333000 & -0.301667000 & 1 & -1.119740000 \\
\hline
\end{tabular}

4.997238000

3.327155000

5.181112000

3.512253000

4.572414000

4.840511000

6.926503000

6.573557000

$-0.836243000$

$-1.344815000$

$-1.235622000$

$-0.787361000$

$-2.654298000$

$-1.624737000$

$-1.675415000$

$-1.178196000$

1.279673000

0.739321000

0.662908000

1.077366000

$-0.822986000$

2.422055000

$-0.801162000$

101771000

$-3.662174000$

(

$-2.058799000$

194115000

4671511000

$-5.064833000$

632746000

$-3.787220000$

3.236707000

291322000

$-6.625861000$

$-5.152502000$

(11259000

$-6.718503000$

6.867686000

4.362651000

3.830191000

$-1.720903000$

$-1.021147000$

0.094783000

.209936000

1.180273000

0.431201000

$-1.119740000$
1.791621000

1.376826000

$-0.673328000$

$-0.960210000$

$-0.980079000$

$-2.015102000$

0.263405000

$-1.096607000$

0.931642000

1.645766000

1.387664000

0.541220000

$-0.571228000$

$-0.732134000$

$-1.035109000$

$-1.571273000$

1.151752000

$-0.073334000$

0.246146000

$-0.564724000$

2.595869000

1.806596000

2.410485000

0.714411000

2.678002000

2.420001000

1.409841000

2.336548000

1.777162000

2.396279000

0.866790000

$-0.427896000$

0.281467000

0.516819000

1.012169000

3.359534000

3.228649000

0.251679000

2.001758000

$-0.262595000$

$-1.062810000$

$-1.881072000$

$-1.323244000$

$-1.721357000$

$-1.496118000$

$-0.563220000$

$-2.311407000$

$-2.544739000$

$-3.494030000$

$-2.637765000$

$-2.882152000$

$-0.941845000$

$-0.132178000$

$-1.043559000$

3.407406000

5.596079000

5.857870000

4.106139000

4.034475000

3.762194000

4.287720000

2.682368000

4.250833000

2.660160000

3.745917000

4.042448000

6.206332000

5.847795000
$-2.886980000$

$-2.440268000$

$-3.786535000$

$-3.220234000$

$-2.922793000$

$-2.659350000$

$-2.139580000$

$-1.037915000$

$-1.678415000$

$-3.936333000$

$-5.000947000$

$-3.022210000$

$-2.861293000$

$-3.224670000$

$-4.282502000$

$-2.665387000$

$-3.317855000$

$-4.492616000$

$-3.441001000$

$-2.818985000$

$-3.749421000$

$-3.755047000$

$-1.433944000$

1.412007000

2.001941000

1.275004000

2.505815000

2.901456000

3.446604000

4.282987000

3.860023000

3.531436000

2.568345000

3.227876000

4.128394000

1.529689000

2.825631000

0.778115000

$-1.042245000$

$-0.495050000$

$-2.038962000$

$-2.285982000$

$-0.739793000$

1.348768000

0.414698000

0.561812000

0.227913000

$-1.717195000$

$-1.131150000$

$-0.898024000$

0.038246000

$-2.880092000$

$-1.944607000$

$-0.096516000$

$-1.243108000$

$-0.854406000$

$-1.910567000$

$-0.726833000$

$-2.594922000$

$-1.537092000$

$-1.151951000$

$-1.500586000$

1.169200000

0.880575000

0.747693000

1.333735000

$-0.465027000$

$-0.291020000$ 


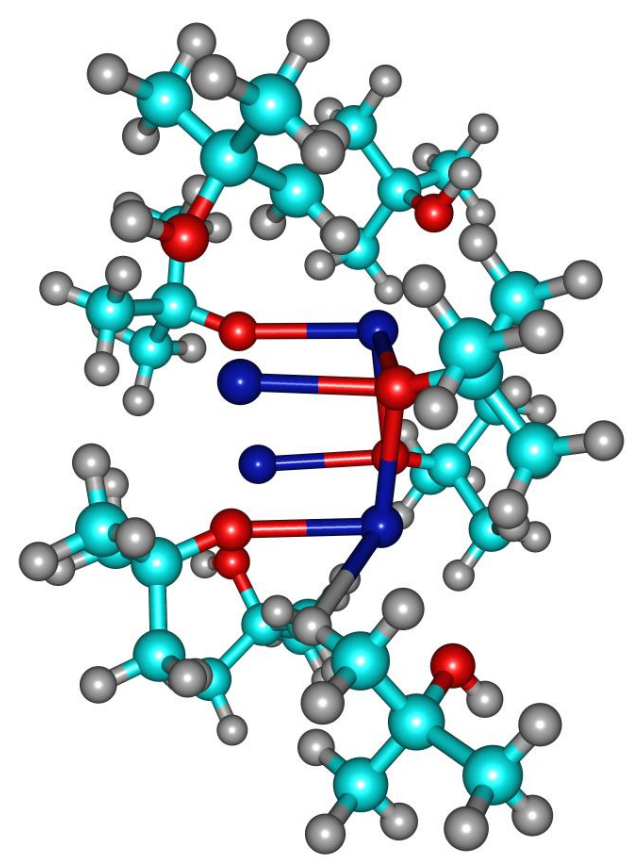

S7·4t-BuOH

\section{basis $=$ def2-SVP}

$E=-4264.462212$

\section{$E($ def2QZVP) $=-4267.150604$}

$\mathbf{G}=\mathbf{- 4 2 6 3 . 6 4 8 6 9 4}($ Temperature $=298.15$ pressure $=24.45)$

\begin{tabular}{|c|c|c|c|c|}
\hline $4-$ & & & & \\
\hline 0.762747000 & 1.733856000 & -1.163632000 & 1 & 5.348808000 \\
\hline-2.247206000 & 1.174795000 & -2.729575000 & 1 & 2.976353000 \\
\hline-1.738831000 & 0.858677000 & -1.486807000 & 8 & 2.843922000 \\
\hline-1.728957000 & 0.563583000 & 1.082889000 & 6 & 4.096581000 \\
\hline 0.611282000 & 1.733660000 & 1.395384000 & 6 & 5.136590000 \\
\hline-0.428625000 & 2.203092000 & 3.519899000 & 1 & 6.102730000 \\
\hline-0.301643000 & 2.694499000 & 4.498417000 & 1 & 3.492065000 \\
\hline 0.802818000 & 2.366072000 & 2.601907000 & 6 & 3.828369000 \\
\hline 0.185643000 & 4.317676000 & 1.866154000 & 1 & 4.739273000 \\
\hline 1.048345000 & 3.872833000 & 2.388751000 & 1 & 3.038729000 \\
\hline 1.204792000 & 4.425346000 & 3.330149000 & 1 & 3.767746000 \\
\hline 1.936609000 & 4.016670000 & 1.751988000 & 1 & 4.670013000 \\
\hline 2.926407000 & 1.854071000 & 2.730647000 & 6 & 4.531299000 \\
\hline 1.846642000 & 0.714660000 & 3.587777000 & 1 & 5.482289000 \\
\hline 2.019983000 & 1.779332000 & 3.353753000 & 1 & 5.292564000 \\
\hline 2.220729000 & 2.294464000 & 4.307434000 & 1 & 4.807954000 \\
\hline-0.618398000 & 1.131749000 & 3.706196000 & 1 & -2.956800000 \\
\hline-1.323757000 & 2.636653000 & 3.042873000 & 8 & -2.822551000 \\
\hline-3.754778000 & 1.498652000 & -2.657267000 & 6 & -4.079097000 \\
\hline-3.925683000 & 2.386750000 & -2.028149000 & 6 & -5.102712000 \\
\hline-4.192762000 & 1.696414000 & -3.649615000 & 1 & -6.070549000 \\
\hline-4.298716000 & 0.652869000 & -2.206296000 & 1 & -3.454797000 \\
\hline-2.063476000 & -0.000362000 & -3.714276000 & 6 & -3.805888000 \\
\hline-2.573837000 & -0.900624000 & -3.332692000 & 1 & -4.717146000 \\
\hline-2.460224000 & 0.215804000 & -4.720026000 & 1 & -3.024125000 \\
\hline-0.989951000 & -0.231064000 & -3.822702000 & 1 & -3.785315000 \\
\hline-1.538896000 & 2.411287000 & -3.329377000 & 1 & -4.680579000 \\
\hline-1.996473000 & 2.744235000 & -4.275157000 & 6 & -4.537604000 \\
\hline-1.585031000 & 3.250912000 & -2.615178000 & 1 & -5.491444000 \\
\hline-0.480055000 & 2.191537000 & -3.549639000 & 1 & -5.262220000 \\
\hline 1.744479000 & -0.623807000 & 1.075980000 & 1 & -4.755644000 \\
\hline 2.339450000 & -0.958135000 & -2.757185000 & 1 & -4.658823000 \\
\hline 1.799955000 & -0.733522000 & -1.507632000 & 8 & -4.019922000 \\
\hline-0.708843000 & -1.639339000 & -1.294319000 & 6 & -4.693125000 \\
\hline-0.604987000 & -1.807084000 & 1.261963000 & 6 & -5.804760000 \\
\hline-1.108142000 & -3.984028000 & 2.135591000 & 1 & -6.330958000 \\
\hline-1.296026000 & -4.580074000 & 3.044078000 & 1 & -3.207337000 \\
\hline-0.831409000 & -2.496624000 & 2.431319000 & 6 & -3.636667000 \\
\hline-2.945895000 & -1.943024000 & 2.555327000 & 1 & -4.081588000 \\
\hline-2.048322000 & -1.924362000 & 3.195280000 & 1 & -2.826059000 \\
\hline-2.276027000 & -2.488603000 & 4.114429000 & 1 & -4.443513000 \\
\hline-1.855986000 & -0.879453000 & 3.493634000 & 1 & -5.990046000 \\
\hline 0.590300000 & -1.353879000 & 3.629026000 & 6 & -5.253694000 \\
\hline 1.283454000 & -2.827758000 & 2.889244000 & 1 & -5.753398000 \\
\hline
\end{tabular}

0.386907000

0.234811000

$-0.246313000$

$-1.987143000$

2.160201000

2.654828000

2.576963000

1.086479000

1.660139000

0.602552000

2.138873000

1.703694000

3.849586000

4.020718000

4.310509000

4.372618000

4.593358000

3.944753000

4.582915000

5.728613000

6.226464000

3.123388000

3.511219000

3.928486000

2.674576000

4.258389000

5.836976000

5.091354000

5.565791000

6.481255000

5.348808000

976353000

6.102730000

3.492065000

.

3.038729000

3.767746000

570013000

5.482289000

5.292564000

07954000

$-2.822551000$

4.079097000

4.443513000

$-5.753398000$
$-2.409186000$

$-2.954286000$

$-4.423145000$

4.072955000

0.277346000

1.155850000

0.130845000

0.504189000

$-2.159800000$

$-1.937753000$

$-2.422109000$

$-3.045718000$

$-1.265585000$

$-2.190130000$

$-1.393117000$

$-0.443945000$

$-1.217735000$

$-1.631727000$

$-2.711620000$

$-2.152089000$

$-2.948413000$

$-2.592391000$

$-3.328523000$

$-4.171098000$

$-3.708715000$

$-4.097712000$

$-3.263696000$

$-3.726142000$

$-4.582618000$

$-1.674768000$

$-1.399516000$

4.065443000

3.349080000

3.077500000

2.709653000

2.474614000

1.023911000

1.905497000

1.639276000

2.169110000

4.580049000

5.169526000

4.313135000

4.133724000

3.547138000

1.832444000

$-3.823898000$

$-3.159411000$

$-2.918430000$

$-2.464048000$

$-2.247983000$

$-0.902775000$

$-1.820147000$

$-1.585377000$

$-2.146948000$

$-4.530421000$

$-5.005000000$

$-4.200722000$

$-4.045934000$

$-3.249534000$

$-1.554257000$

0.809924000

1.320588000

2.450441000

1.953080000

2.793805000

2.579294000

3.210913000

4.095680000

3.553253000

3.637360000

2.747220000

3.314290000

4.207932000
3.376315000

4.322325000

1.606914000

1.476239000

$-3.665879000$

$-3.218525000$

$-4.676069000$

$-3.778494000$

$-3.453171000$

$-3.677482000$

$-4.410654000$

$-2.797094000$

$-2.673334000$

$-2.099208000$

$-3.666820000$

$-2.157599000$

2.775248000

2.188235000

1.493269000

0.655952000

0.083284000

$-0.120208000$

0.601855000

0.031696000

1.208517000

3.127591000

3.178361000

2.512232000

2.011450000

1.303668000

$-0.050968000$

$-2.414594000$

$-1.777474000$

$-1.132940000$

$-2.186724000$

$-1.716489000$

$-0.767341000$

$-0.196152000$

0.360202000

0.526270000

0.394028000

$-1.030255000$

$-0.351282000$

0.171259000

$-2.885426000$

$-2.763477000$

$-2.727223000$

$-2.036633000$

$-1.387635000$

$-2.423560000$

$-1.947664000$

$-0.869214000$

$-0.366657000$

0.203124000

0.338420000

0.031208000

$-1.439350000$

$-0.700385000$

$-0.175096000$

$-3.179571000$

$-2.935251000$

2.712020000

2.194756000

1.624299000

0.705736000

0.230202000

0.037246000

0.831148000

0.353423000

1.493077000

3.420044000

3.340708000

2.749360000

2.348005000 


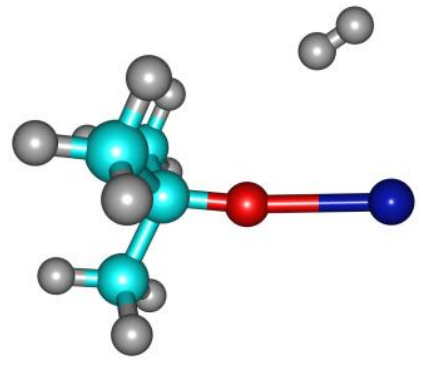

basis = def2-SVP

$E=-833.830563570$

\section{$E($ def2QZVP $)=-834.2243629$}

$\mathbf{G}=\mathbf{- 8 3 3 . 7 5 4 5 3 7}($ Temperature $=\mathbf{2 9 8 . 1 5}$ pressure $=\mathbf{2 4 . 4 5})$

$\begin{array}{llll}19 & -2.561482000 & 0.189646000 & 0.000012000\end{array}$

$\begin{array}{llll}8 & -0.231197000 & 0.113134000 & 0.000226000\end{array}$

$\begin{array}{llll}1 & -1.190266000 & -2.163763000 & 0.000073000\end{array}$

$\begin{array}{llll}1 & -1.725905000 & -2.702054000 & -0.000234000\end{array}$

$\begin{array}{llll}6 & 1.137952000 & 0.037196000 & 0.000015000\end{array}$

$\begin{array}{llll}6 & 1.646237000 & -0.714167000 & 1.248853000\end{array}$

$\begin{array}{llll}6 & 1.646040000 & -0.712018000 & -1.250202000\end{array}$

$\begin{array}{llll}6 & 1.763657000 & 1.447540000 & 0.001144000\end{array}$

$\begin{array}{llll}1 & 1.296539000 & -0.194473000 & -2.158577000\end{array}$

$\begin{array}{llll}1 & 1.234909000 & -1.736896000 & 1.263733000\end{array}$

$\begin{array}{llll}1 & 1.425027000 & 2.001765000 & 0.891838000\end{array}$

$\begin{array}{llll}1 & 2.866301000 & 1.434132000 & 0.001041000\end{array}$

$\begin{array}{llll}1 & 1.424881000 & 2.003263000 & -0.888562000\end{array}$

$1 \quad 2.745612000-0.783271000-1.294885000$

$\begin{array}{llll}1 & 1.234672000 & -1.734703000 & -1.266775000\end{array}$

$\begin{array}{llll}1 & 2.745816000 & -0.785470000 & 1.293295000\end{array}$

$\begin{array}{llll}1 & 1.296836000 & -0.198192000 & 2.158160000\end{array}$

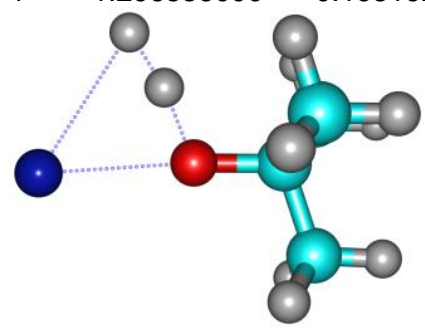

$\mathrm{TS}_{1}$

basis $=$ def2-SVP

$\mathrm{E}=-833.819190625$

$\mathrm{E}($ def2QZVP $)=-834.207042290$

$v=1317 \mathrm{~cm}^{-1}$

$\mathbf{G}=\mathbf{- 8 3 3 . 7 4 2 3 3 6}($ Temperature $=\mathbf{4 8 3} .15$ pressure $=39.64)$

$\begin{array}{lrrr}19 & -2.616692000 & 0.184386000 & 0.083246000 \\ 8 & -0.206861000 & 0.004208000 & -0.010956000 \\ 1 & -0.670322000 & -1.210890000 & -0.041797000 \\ 1 & -1.182025000 & -2.043090000 & -0.059532000 \\ 6 & 1.190520000 & 0.026007000 & -0.001569000 \\ 6 & 1.717366000 & -0.675065000 & 1.259529000 \\ 6 & 1.735684000 & -0.687795000 & -1.247765000 \\ 6 & 1.663044000 & 1.483404000 & -0.005933000 \\ 1 & 1.346782000 & -0.203025000 & -2.157113000 \\ 1 & 1.384356000 & -1.724431000 & 1.279947000 \\ 1 & 1.274227000 & 2.009586000 & 0.880145000 \\ 1 & 2.761971000 & 1.558574000 & 0.001707000 \\ 1 & 1.286916000 & 1.999653000 & -0.903217000 \\ 1 & 2.836238000 & -0.666627000 & -1.286846000 \\ 1 & 1.411816000 & -1.740102000 & -1.258735000 \\ 1 & 2.817619000 & -0.662399000 & 1.310451000 \\ 1 & 1.323225000 & -0.175598000 & 2.158672000\end{array}$

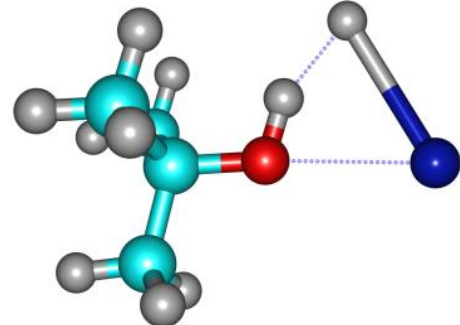

basis = def2-SVP

$E=-833.825840999$

$E($ def2QZVP $)=-834.2135484$

$\mathbf{G}=\mathbf{- 8 3 3 . 7 4 4 8 8 2}($ Temperature $=\mathbf{4 8 3} .15$ pressure $=39.64)$

$\begin{array}{llll}19 & 2.713781000 & 0.088191000 & -0.000038000\end{array}$

$\begin{array}{llll}8 & 0.181747000 & -0.009683000 & -0.000850000\end{array}$

$1 \quad 0.494008000-0.969333000 \quad-0.000596000$

$\begin{array}{llll}1 & 1.469672000 & -2.155476000 & -0.000022000\end{array}$

$\begin{array}{llll}6 & -1.242392000 & 0.025932000 & 0.000014000\end{array}$

$\begin{array}{llll}6 & -1.761145000 & -0.676468000 & -1.254168000\end{array}$

$\begin{array}{llll}6 & -1.759485000 & -0.671706000 & 1.257528000\end{array}$

$\begin{array}{llll}6 & -1.642764000 & 1.495488000 & -0.002528000\end{array}$

$\begin{array}{llll}1 & -1.363904000 & -0.176373000 & 2.157133000\end{array}$

$1-1.435515000 \quad-1.728141000 \quad-1.264443000$

$1-1.245024000 \quad 1.998972000 \quad-0.896678000$

$\begin{array}{llll}1 & -2.737302000 & 1.601867000 & -0.001979000\end{array}$

$\begin{array}{llll}1 & -1.243787000 & 2.002433000 & 0.889112000\end{array}$

$\begin{array}{llll}1 & -2.858642000 & -0.649447000 & 1.300865000\end{array}$

$\begin{array}{llll}1 & -1.433648000 & -1.723273000 & 1.271439000\end{array}$

$\begin{array}{llll}1 & -2.860352000 & -0.654198000 & -1.296208000\end{array}$

$\begin{array}{llll}1 & -1.366609000 & -0.184682000 & -2.156171000\end{array}$

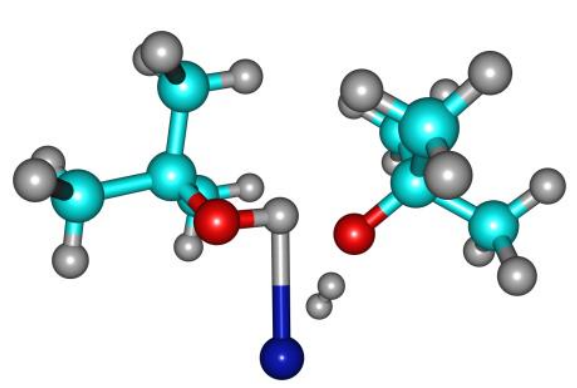

3

basis $=$ def2-SVP

$E=-1067.25106$

$E($ def2QZVP $)=-1067.934884$

$\mathbf{G}=\mathbf{- 1 0 6 7 . 0 6 3 8 4}$ (Temperature $=\mathbf{2 9 8 . 1 5}$ pressure $=\mathbf{2 4 . 4 5}$ )

$\begin{array}{llll}19 & -0.154732000 & 2.479080000 & -0.244778000\end{array}$

$\begin{array}{llll}8 & 1.096324000 & 0.383753000 & 0.125517000\end{array}$

$\begin{array}{llll}6 & 3.475324000 & 0.197942000 & -0.056178000\end{array}$

$\begin{array}{llll}1 & 4.326497000 & -0.492731000 & -0.168656000\end{array}$

$\begin{array}{llll}6 & 2.124295000 & -0.533724000 & -0.034422000\end{array}$

$\begin{array}{llll}1 & 1.964328000 & -0.597346000 & -2.203984000\end{array}$

$\begin{array}{llll}6 & 1.951216000 & -1.301331000 & -1.356141000\end{array}$

$\begin{array}{llll}1 & 2.744733000 & -2.048879000 & -1.516653000\end{array}$

$\begin{array}{llll}1 & 0.979866000 & -1.820987000 & -1.367040000\end{array}$

$\begin{array}{llll}1 & 1.181390000 & -2.097716000 & 1.155001000\end{array}$

$\begin{array}{llll}1 & 2.229731000 & -0.999337000 & 2.086425000\end{array}$

$\begin{array}{llll}6 & 2.130544000 & -1.538617000 & 1.130396000\end{array}$

$\begin{array}{llll}1 & 2.954143000 & -2.267120000 & 1.056890000\end{array}$

$\begin{array}{llll}1 & 3.610863000 & 0.761649000 & 0.881061000\end{array}$

$\begin{array}{llll}1 & 3.502054000 & 0.915631000 & -0.892016000\end{array}$

$1 \quad-0.203866000 \quad 0.042216000 \quad-0.375363000$

$\begin{array}{llll}8 & -1.185484000 & 0.123115000 & -0.769126000\end{array}$

$\begin{array}{llll}6 & -2.108119000 & -0.610277000 & 0.009836000\end{array}$

$\begin{array}{llll}6 & -1.855806000 & -2.109479000 & -0.172715000\end{array}$

$\begin{array}{lll}-2.590122000 & -2.716627000 & 0.378473000\end{array}$

$\begin{array}{lll}-3.601192000 & -0.494292000 & -1.554544000\end{array}$

$\begin{array}{lll}-3.507451000 & -0.254390000 & -0.484359000\end{array}$

$\begin{array}{lll}-4.281190000 & -0.809475000 & 0.066852000\end{array}$

$\begin{array}{lll}-3.696455000 & 0.823053000 & -0.356236000\end{array}$

$\begin{array}{lll}-2.180664000 & 0.834974000 & 1.641024000\end{array}$ 


$\begin{array}{ll}1 & -0.921464000 \\ 6 & -1.955136000 \\ 1 & -2.636757000 \\ 1 & -0.850429000 \\ 1 & -1.914928000 \\ 1 & 0.632936000 \\ 1 & 0.874514000\end{array}$

$-0.420186000$

$-0.234128000$

$-0.814774000$

$-2.373001000$

$-2.371626000$

2.333005000

1.760137000

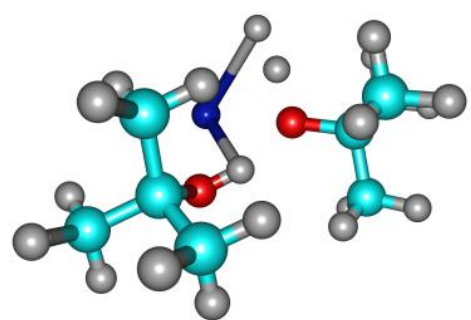

$\mathrm{TS}_{2}$

basis = def2-SVP

$E=-1067.233182$

$E($ def2QZVP) $=-1067.912752$

$\mathrm{v}=1349 \mathrm{~cm}^{-1}$

$\mathbf{G}=\mathbf{- 1 0 6 7 . 0 4 4 2 2 4}($ Temperature $=\mathbf{4 8 3 . 1 5}$ pressure $=39.64)$

$\begin{array}{lll}\mathrm{G} & -0.265406000 \quad 2.530815000\end{array}$

$8 \quad 1.179341000 \quad 0.411915000$

3.576148000

4.431209000

2.242375000

0.248325000

$-0.437500000$

$-0.504760000$

2.134263000

2.094172000

$-0.504032000$

$-1.227167000$

$-1.970382000$

$1.124000000-1.746865000$

1.229241000

2.255443000

$-2.069525000$

$-1.005803000$

2.183536000

3.003162000

$-1.520169000$

$-2.252431000$

0.787303000

0.984339000

3.622768000

$-0.350162000$

0.000133000

0.172224000

$-1.284202000$

$-2.179371000$

$-0.642756000$

$-2.112849000$

$-2.776419000$

$-0.480097000$

$-0.290111000$

$-0.889578000$

0.774475000

0.706699000

$-0.503408000$

$-0.341077000$

$-0.985842000$

$-2.362335000$

$-2.310516000$

1.616621000

1.009957000

$-0.121408000$

0.085082000

0.005940000

$-0.097519000$

$-0.036888000$

$-2.205970000$

$-1.376486000$

$-1.525524000$

$-1.423070000$

1.083642000

2.081102000

1.110417000

1.046376000

0.960101000

$-0.811838000$

$-0.435875000$

$-0.741805000$

0.008675000

$-0.273461000$

0.246339000

$-1.537512000$

$-0.458312000$

0.073993000

$-0.272873000$

1.716177000

1.800484000

1.498052000

2.114711000

0.067029000

$-1.354219000$

1.991690000

1.060401000

1.175666000

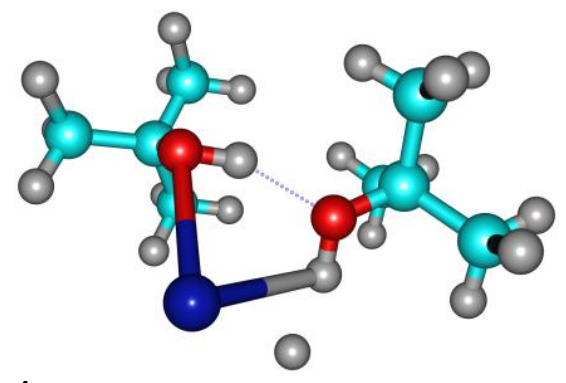

4

basis = def2-SVP

$E=-1067.236967$

$E($ def2QZVP) $=-1067.916919$

$\mathbf{G}=\mathbf{- 1 0 6 7 . 0 4 5 1 9 7}$ (Temperature $=298.15$ pressure $=24.45$ )

$\begin{array}{llll}19 & -0.293256000 & 2.555067000 & -0.117014000\end{array}$

$\begin{array}{llll}8 & 1.293861000 & 0.435504000 & 0.044915000\end{array}$

$6 \quad 3.683095000$

4.526632000

2.355716000

2.475710000

2.314016000

3.091576000

1.333443000

1.170885000

2.078836000

2.122574000

2.931387000

3.691480000

3.833191000

$-0.414498000$

$-1.324170000$

$-2.261636000$

$-2.083277000$

$-2.818983000$

$-3.788463000$

$-3.642933000$

$-4.427524000$

$-3.758606000$

$-2.171965000$

$-0.988528000$

$-2.021317000$

$-2.708345000$

$-1.076213000$

$-2.204712000$

0.968972000

1.272640000

0.196760000

$-0.505288000$

$-0.513298000$

$-0.296260000$

$-1.090170000$

$-1.856445000$

$-1.552219000$

$-2.127796000$

$-1.175994000$

$-1.610123000$

$-2.355641000$

0.638406000

1.003868000

$-0.077010000$

0.200732000

$-0.659384000$

$-2.078137000$

$-2.766076000$

$-0.116472000$

$-0.121466000$

$-0.743092000$

0.908043000

0.402579000

$-0.921352000$

$-0.616737000$

$-1.292773000$

$-2.454345000$

$-2.088171000$

1.807375000

0.887837000
0.225708000

0.148281000

$-0.032267000$

$-2.186400000$

$-1.441666000$

$-1.574756000$

$-1.636094000$

0.809043000

2.016757000

1.005942000

0.983411000

1.233867000

$-0.507194000$

$-0.399046000$

$-0.637519000$

0.012340000

$-0.522718000$

$-0.081030000$

$-1.423879000$

$-0.333273000$

0.121110000

0.039023000

1.908887000

1.752818000

1.519883000

2.050177000

$-0.281938000$

$-1.616177000$

2.039375000

0.964180000

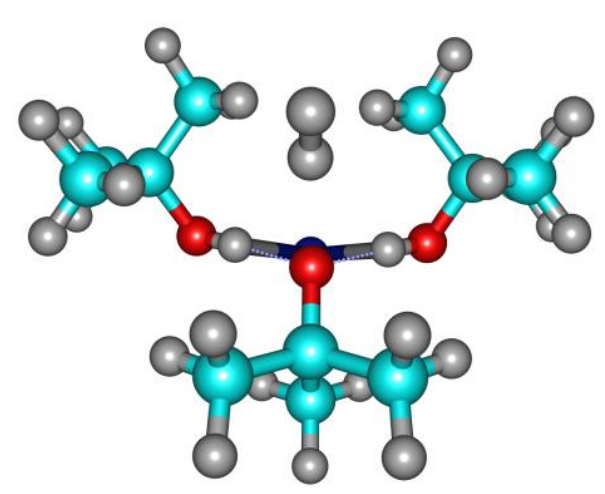

5

basis = def2-SVP

$E=-1300.670072$

$E($ def2QZVP) $=-1301.644758$

$\mathbf{G}=\mathbf{- 1 3 0 0 . 3 6 9 6 7 9}$ (Temperature $=298.15$ pressure $=24.45$ )

$\begin{array}{llll}1 & 1.347177000 & 0.270747000 & 0.095218000\end{array}$

$\begin{array}{lllll}19 & 0.029160000 & -0.777009000 & 1.970133000\end{array}$

$\begin{array}{llll}6 & -2.893902000 & -0.963198000 & -0.361222000\end{array}$

$\begin{array}{llll}8 & -2.096474000 & -0.247096000 & 0.566147000\end{array}$

$\begin{array}{llll}6 & -3.528658000 & 0.012187000 & -1.353819000\end{array}$

$\begin{array}{lll}-2.745667000 & 0.552285000 & -1.908395000\end{array}$

$\begin{array}{lll}-4.164818000 & -0.514507000 & -2.081214000\end{array}$

$\begin{array}{lll}-4.147332000 & 0.749943000 & -0.820167000\end{array}$

$\begin{array}{lll}-3.974045000 & -1.679568000 & 0.441628000\end{array}$

$\begin{array}{lll}-4.654708000 & -2.238613000 & -0.217139000\end{array}$

$\begin{array}{lll}-3.519349000 & -2.388317000 & 1.151247000\end{array}$

$\begin{array}{lll}-4.563709000 & -0.948274000 & 1.014918000\end{array}$

$-2.023925000 \quad-1.974610000-1.110961000$

$\begin{array}{lll}-1.547370000 & -2.674045000 & -0.404379000\end{array}$

$\begin{array}{lll}-2.616843000 & -2.565180000 & -1.825803000\end{array}$

$-1.234272000-1.450909000-1.671337000$

$\begin{array}{lll}0.011635000 & 0.873266000 & -0.249445000\end{array}$

$\begin{array}{lll}0.016458000 & 2.248542000 & 0.031790000\end{array}$

$\begin{array}{lll}1.255969000 & 2.897357000 & -0.595856000\end{array}$

$\begin{array}{lll}2.166824000 & 2.423855000 & -0.197739000\end{array}$

$\begin{array}{lll}1.244412000 & 2.756075000 & -1.688086000\end{array}$

$\begin{array}{lll}1.305386000 & 3.977762000 & -0.387546000\end{array}$

$\begin{array}{lll}-1.287070000 & 2.758172000 & -1.631035000\end{array}$

$\begin{array}{lll}-1.249609000 & 2.897541000 & -0.539150000\end{array}$ 


$\begin{array}{rrrr}1 & -1.288819000 & 3.977731000 & -0.327602000 \\ 1 & 0.057303000 & 3.548571000 & 1.812895000 \\ 6 & 0.050002000 & 2.478462000 & 1.553700000 \\ 1 & 0.960586000 & 2.030780000 & 1.988904000 \\ 1 & -0.843382000 & 2.034708000 & 2.027281000 \\ 1 & -2.142223000 & 2.424829000 & -0.100731000 \\ 8 & 2.151571000 & -0.190023000 & 0.556772000 \\ 6 & 2.870591000 & -0.979336000 & -0.375800000 \\ 6 & 3.375330000 & -0.092471000 & -1.515187000 \\ 1 & 2.526230000 & 0.385575000 & -2.027723000 \\ 1 & 4.031344000 & 0.698299000 & -1.120055000 \\ 1 & 3.941996000 & -0.676299000 & -2.256216000 \\ 1 & 4.673664000 & -0.807140000 & 0.807130000 \\ 6 & 4.044226000 & -1.599118000 & 0.374027000 \\ 1 & 4.664680000 & -2.213251000 & -0.295179000 \\ 1 & 2.472560000 & -2.699451000 & -1.672321000 \\ 6 & 1.953271000 & -2.071012000 & -0.933013000 \\ 1 & 1.079331000 & -1.614872000 & -1.423694000 \\ 1 & 1.598220000 & -2.730105000 & -0.123026000 \\ 1 & 3.682019000 & -2.238438000 & 1.194185000 \\ 1 & -1.318168000 & 0.240833000 & 0.091818000 \\ 1 & -0.076603000 & 0.524034000 & -3.436050000 \\ 1 & -0.063535000 & 0.630549000 & -2.685302000\end{array}$

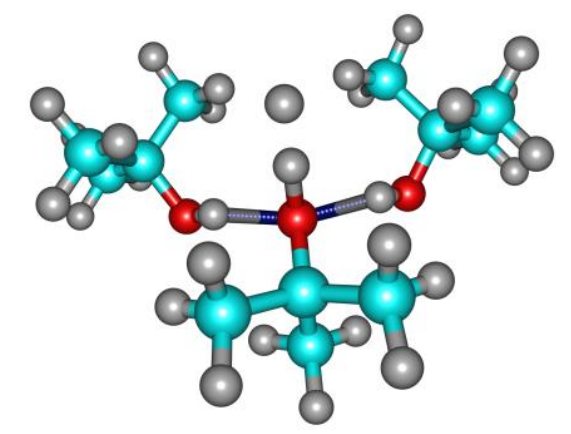

$\mathrm{TS}_{3}$

basis $=$ def2-SVP

$E=-1300.644416$$$
\begin{aligned}
& E(\text { def2QZVP })=-1301.615986 \\
& v=718 \mathrm{~cm}^{-1}
\end{aligned}
$$

$\mathbf{G}=\mathbf{- 1 3 0 0 . 3 4 2 5 6}($ Temperature $=\mathbf{4 8 3} .15$ pressure $=\mathbf{3 9 . 6 4})$

$\begin{array}{llll}19 & 0.000123000 & -0.886421000 & 1.720627000\end{array}$

$\begin{array}{llll}6 & -3.027619000 & -1.005865000 & -0.351523000\end{array}$

$\begin{array}{llll}8 & -2.278329000 & -0.210110000 & 0.570168000\end{array}$

$\begin{array}{llll}6 & -3.617525000 & -0.096353000 & -1.426406000\end{array}$

$\begin{array}{llll}1 & -2.809407000 & 0.409482000 & -1.979128000\end{array}$

$\begin{array}{llll}1 & -4.217103000 & -0.672498000 & -2.146378000\end{array}$

$\begin{array}{llll}1 & -4.261965000 & 0.668204000 & -0.966804000\end{array}$

$\begin{array}{llll}6 & -4.126273000 & -1.677997000 & 0.458823000\end{array}$

$\begin{array}{llll}1 & -4.758559000 & -2.305102000 & -0.185835000\end{array}$

$\begin{array}{llll}1 & -3.691089000 & -2.315935000 & 1.243522000\end{array}$

$\begin{array}{llll}1 & -4.759853000 & -0.918699000 & 0.940834000\end{array}$

$\begin{array}{llll}6 & -2.100902000 & -2.043700000 & -0.985223000\end{array}$

$\begin{array}{llll}1 & -1.683343000 & -2.717330000 & -0.217905000\end{array}$

$1-2.639783000 \quad-2.667016000-1.714101000$

$1 \quad-1.274775000 \quad-1.541306000 \quad-1.514187000$

$8-0.000181000$

$6-0.000394000$

1.258701000

2.156543000

1.273750000

1.304227000

$-1.273577000$

$-1.259210000$

$-1.305019000$

$-0.001233000$

$-0.000937000$

0.901804000

$-0.903798000$

$-2.157239000$

2.278316000

3.027955000
$0.987537000-0.289221000$

$2.376251000 \quad 0.040199000$

$3.030325000-0.530915000$

$2.534991000-0.129101000$

$2.941684000-1.627391000$

$4.097835000-0.267536000$

$2.941438000-1.628218000$

$3.030142000-0.531739000$

$4.097660000-0.268445000$

$3.534128000 \quad 1.887483000$

$2.482484000 \quad 1.564663000$

$2.008778000 \quad 1.986351000$

$2.008421000 \quad 1.985698000$

$2534729000-0.130444000$

$\begin{array}{ll}-0.209869000 & 0.570075000\end{array}$

$-1.005427000-0.351507000$

$\begin{array}{rr}6 & 3.617950000 \\ 1 & 2.809867000 \\ 1 & 4.262177000 \\ 1 & 4.217765000 \\ 1 & 4.759879000 \\ 6 & 4.126531000 \\ 1 & 4.759065000 \\ 1 & 2.640726000 \\ 6 & 2.101561000 \\ 1 & 1.275485000 \\ 1 & 1.683915000 \\ 1 & 3.691268000 \\ 1 & -1.608961000 \\ 1 & 0.000367000 \\ 1 & 0.000078000\end{array}$

$-0.095718000$

0.410047000

0.668892000

$-0.671700000$

$-0.918087000$

$-1.677442000$

$-2.304388000$

$-2.666564000$

$-2.043370000$

$-1.541076000$

$-2.717097000$

$-2.315526000$

0.308040000

0.628595000

0.832754000

$-1.426172000$

$-1.979009000$

$-0.966361000$

$-2.146077000$

0.941267000

0.459042000

$-0.185526000$

$-1.714266000$

$-0.985493000$

$-1.514627000$

$-0.218310000$

1.243579000

0.066128000

$-2.542671000$

$-1.421082000$

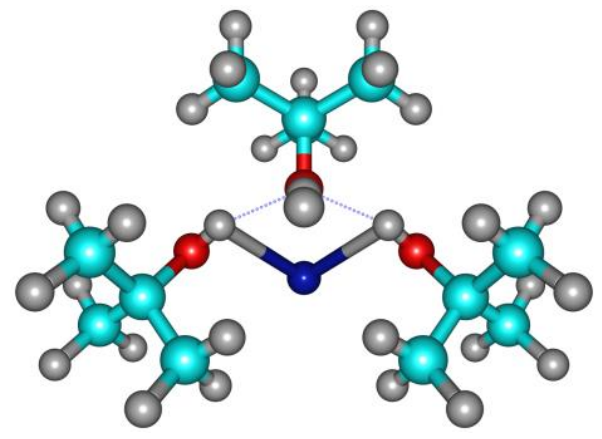

6

basis = def2-SVP

$E=-1300.644507$

$E($ def2QZVP) $=-1301.616543$

$\mathrm{G}=\mathbf{- 1 3 0 0 . 3 4 0 9 1 9}$ (Temperature $=298.15$ pressure $=24.45$ )

$1 \quad 1.647769000 \quad 0.318619000 \quad 0.055441000$

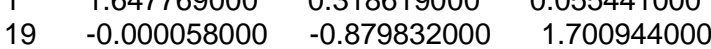

$\begin{array}{llll}6 & -3.040909000 & -1.022960000 & -0.345835000\end{array}$

$\begin{array}{llll}8 & -2.292215000 & -0.215804000 & 0.568280000\end{array}$

$\begin{array}{llll}6 & -3.648392000 & -0.121801000 & -1.417486000\end{array}$

$1 \quad-2.849618000 \quad 0.387694000 \quad-1.980528000$

$\begin{array}{llll}1 & -4.250609000 & -0.705308000 & -2.129178000\end{array}$

$\begin{array}{llll}1 & -4.293998000 & 0.639370000 & -0.953984000\end{array}$

$\begin{array}{llll}6 & -4.124872000 & -1.703709000 & 0.476626000\end{array}$

$\begin{array}{llll}1 & -4.754097000 & -2.341690000 & -0.160223000\end{array}$

$\begin{array}{llll}1 & -3.675961000 & -2.332409000 & 1.261033000\end{array}$

$\begin{array}{llll}1 & -4.764057000 & -0.949777000 & 0.959595000\end{array}$

$\begin{array}{llll}6 & -2.107744000 & -2.052129000 & -0.983696000\end{array}$

$\begin{array}{lll}-1.678767000 & -2.720673000 & -0.218237000\end{array}$

$\begin{array}{lll}-2.645403000 & -2.681883000 & -1.707885000\end{array}$

$\begin{array}{lll}-1.290330000 & -1.542422000 & -1.519404000\end{array}$

$\begin{array}{lll}-0.000014000 & 1.016128000 & -0.284274000\end{array}$

$\begin{array}{lll}-0.000082000 & 2.410486000 & 0.036160000\end{array}$

$\begin{array}{lll}1.259464000 & 3.057325000 & -0.539361000\end{array}$

$\begin{array}{lll}2.156770000 & 2.562563000 & -0.135597000\end{array}$

$\begin{array}{lll}1.272672000 & 2.962455000 & -1.635279000\end{array}$

$\begin{array}{lll}1.306269000 & 4.125913000 & -0.281279000\end{array}$

$\begin{array}{lll}-1.272367000 & 2.962415000 & -1.635652000\end{array}$

$\begin{array}{lll}-1.259469000 & 3.057305000 & -0.539732000\end{array}$

$\begin{array}{lll}-1.306347000 & 4.125898000 & -0.281684000\end{array}$

$\begin{array}{lll}-0.000405000 & 3.568284000 & 1.878972000\end{array}$

$\begin{array}{lll}-0.000320000 & 2.515971000 & 1.559213000\end{array}$

$\begin{array}{lll}0.902295000 & 2.043090000 & 1.981532000\end{array}$

$\begin{array}{lll}-0.903038000 & 2.043026000 & 1.981244000\end{array}$

$\begin{array}{lll}-2.156889000 & 2.562555000 & -0.136212000\end{array}$

$\begin{array}{lll}2.292108000 & -0.215922000 & 0.568318000\end{array}$

$\begin{array}{lll}3.041011000 & -1.022868000 & -0.345806000\end{array}$

$3.648661000 \quad-0.121472000-1.417163000$

$\begin{array}{lll}2.849969000 & 0.388105000 & -1.980249000\end{array}$

$\begin{array}{lll}4.294153000 & 0.639631000 & -0.953390000\end{array}$

$\begin{array}{lll}4.251029000 & -0.704816000 & -2.128861000\end{array}$

$\begin{array}{lll}4.763885000 & -0.949880000 & 0.960003000\end{array}$

$\begin{array}{lll}4.124841000 & -1.703738000 & 0.476731000\end{array}$

$\begin{array}{lll}4.754232000 & -2.341544000 & -0.160128000\end{array}$

$2.645846000 \quad-2.681575000 \quad-1.708221000$

$\begin{array}{lll}2.645846000 & -2.681575000 & -1.708221000 \\ 2.108014000 & -2.051955000 & -0.984042000\end{array}$ 
$\begin{array}{rrr}1.290696000 & -1.542182000 & -1.519830000 \\ 1.678896000 & -2.720622000 & -0.218773000 \\ 3.675797000 & -2.332642000 & 1.260901000 \\ -1.647798000 & 0.318659000 & 0.055420000 \\ 0.000180000 & 0.631854000 & -2.579027000 \\ 0.000076000 & 0.866167000 & -1.360705000\end{array}$

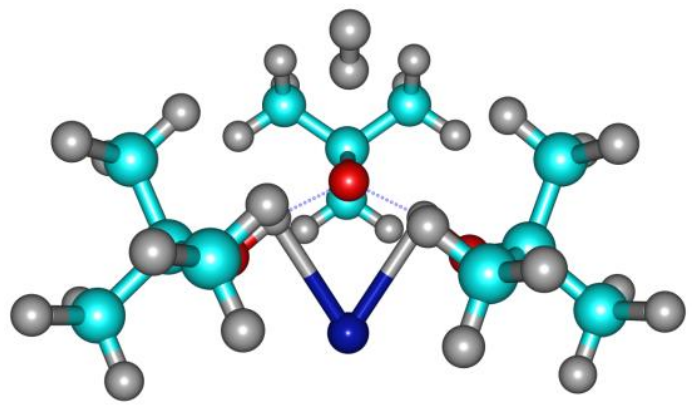

$5^{\star}$

$G=-1300.368846$ (Temperature $=483.15$ pressure $=39.64$, def2SVP)

Thermal correction to $\mathrm{G}=\mathbf{0 . 3 0 1 2 0 3}$ (def2SVP)

$E=\mathbf{- 1 3 0 1 . 6 4 4 7 4 6 2 3}$ (def2QZVP)

$\begin{array}{llll}1 & 1.316920000 & 0.239641000 & 0.090471000\end{array}$

$\begin{array}{lllll}19 & -0.027692000 & -0.776323000 & 1.970756000\end{array}$

$\begin{array}{llll}6 & -2.870861000 & -0.980283000 & -0.375356000\end{array}$

$\begin{array}{llll}8 & -2.155224000 & -0.190923000 & 0.559906000\end{array}$

$\begin{array}{llll}6 & -3.378054000 & -0.092014000 & -1.512565000\end{array}$

$\begin{array}{llll}1 & -2.530400000 & 0.390089000 & -2.023633000\end{array}$

$\begin{array}{llll}1 & -3.942688000 & -0.675686000 & -2.255281000\end{array}$

$1 \quad-4.036676000 \quad 0.695613000-1.115512000$

$\begin{array}{llll}6 & -4.042844000 & -1.606456000 & 0.371688000\end{array}$

$\begin{array}{llll}1 & -4.660366000 & -2.221029000 & -0.299830000\end{array}$

$\begin{array}{llll}1 & -3.679104000 & -2.246673000 & 1.190457000\end{array}$

$1-4.675740000 \quad-0.818060000 \quad 0.806288000$

$-2.066910000$

$\begin{array}{llll}1 & -1.593758000 & -2.728231000 & -0.127171000\end{array}$

$\begin{array}{llll}1 & -1.075668000 & -1.605959000 & -1.422240000\end{array}$

$1-0.051298000$

$8-0.014311000$

$6-0.017627000$

1.246505000

2.140671000

1.279878000

1.286715000

$-1.251177000$

$-1.259038000$

$-1.307337000$

$-0.051448000$

$-0.045745000$

0.848883000

$-0.955142000$

$-2.168772000$

2.095435000

2.895015000

3.533497000

2.752521000

4.150895000

4.171669000

4.560742000

3.972268000

4.654532000

2.621674000

2.026717000

1.239404000

1.547147000

3.514992000

0.060903000

$-1.350960000$
.640395000

0.872228000

2.247967000

2.895801000

2.423587000

2.754701000

3.976320000

2.753475000

2.896307000

3.977042000

3.550874000

2.480369000

2.036558000

2.033949000

2.423748000

$-0.248106000$

$-0.962644000$

0.014501000

0.556287000

0.750698000

$-0.510959000$

$-0.951861000$

$-1.681499000$

$-2.239575000$

$-2.561547000$

$-1.971822000$

$-1.446387000$

$-2.672123000$

$-2.391475000$

0.534404000

0.270783000
$-0.246701000$

0.032097000

$-0.544505000$

$-0.108808000$

$-1.636298000$

$-0.334814000$

$-1.684349000$

$-0.592281000$

1.811330000

1.553724000

2.024861000

1.992703000

$-0.190537000$

0.564642000

$-0.362068000$

$-1.350495000$

$-1.906238000$

$-0.813218000$

$-2.077020000$

1.019082000

0.442450000

$-0.215469000$

$-1.830531000$

$-1.116691000$

$-0.413032000$

1.149195000

$-3.435299000$

0.099470000
$-0.385388000$

$-1.678748000$

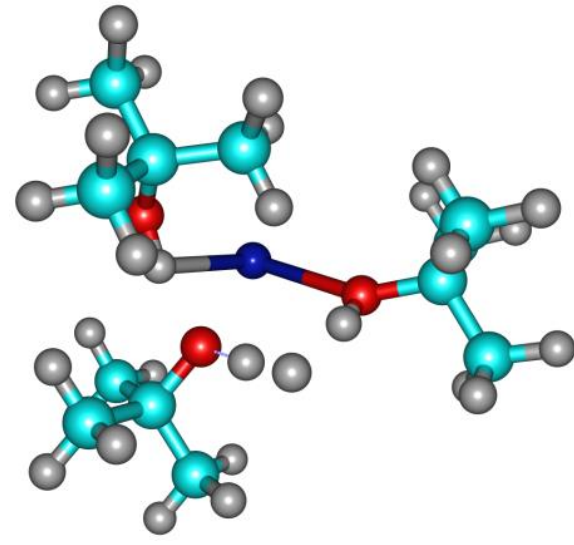

$\mathrm{TS}_{4}$

$G=-1300.343682$ (Temperature $=483.15$ pressure $=39.64$, def2SVP)

$v=1336 \mathrm{~cm}^{-1}$

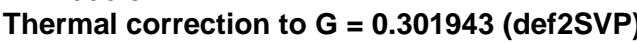

$E=-1301.617297$ (def2QZVP)

$\begin{array}{llll}1 & -1.494771000 & 0.663688000 & 0.723379000\end{array}$

$\begin{array}{llll}19 & -0.238790000 & 0.042077000 & -1.898647000\end{array}$

$\begin{array}{llll}6 & 1.534180000 & -2.312122000 & 0.235954000\end{array}$

$\begin{array}{llll}8 & 1.821449000 & -1.251534000 & -0.671164000\end{array}$

$\begin{array}{lll}2.221773000 & -2.032098000 & 1.571883000\end{array}$

$\begin{array}{lll}1.830242000 & -1.101490000 & 2.012574000\end{array}$

$\begin{array}{lll}2.049570000 & -2.850244000 & 2.286835000\end{array}$

$\begin{array}{lll}3.306548000 & -1.919826000 & 1.423219000\end{array}$

$2.076787000 \quad-3.588514000 \quad-0.392212000$

$\begin{array}{lll}1.886725000 & -4.457620000 & 0.254282000\end{array}$

$\begin{array}{lll}1.598253000 & -3.769619000 & -1.366954000\end{array}$

$3.161735000 \quad-3.499527000 \quad-0.552298000$

$\begin{array}{lll}0.019822000 & -2.408143000 & 0.437658000\end{array}$

$\begin{array}{lll}-0.482779000 & -2.667188000 & -0.510378000\end{array}$

$\begin{array}{lll}-0.241756000 & -3.183866000 & 1.173157000\end{array}$

$\begin{array}{lll}-0.378054000 & -1.447252000 & 0.801444000\end{array}$

$\begin{array}{lll}0.260213000 & 1.034710000 & 1.185710000\end{array}$

$\begin{array}{lll}0.978666000 & 1.066725000 & 0.160456000\end{array}$

$\begin{array}{lll}1.950980000 & 2.089010000 & 0.212029000\end{array}$

$\begin{array}{lll}1.264793000 & 3.439896000 & 0.435804000\end{array}$

$\begin{array}{lll}0.536909000 & 3.633425000 & -0.367995000\end{array}$

$\begin{array}{lll}0.723727000 & 3.443447000 & 1.393990000\end{array}$

$\begin{array}{lll}1.994133000 & 4.264529000 & 0.448265000\end{array}$

$\begin{array}{lll}2.401581000 & 1.752187000 & 2.312238000\end{array}$

$\begin{array}{lll}2.935914000 & 1.804105000 & 1.350992000\end{array}$

$\begin{array}{lll}3.708501000 & 2.584912000 & 1.426407000\end{array}$

$3.510544000 \quad 2.844783000 \quad-1.127100000$

$\begin{array}{lll}2.700718000 & 2.098893000 & -1.122803000\end{array}$

$\begin{array}{lll}2.015394000 & 2.346867000 & -1.950731000\end{array}$

$\begin{array}{lll}3.143481000 & 1.108558000 & -1.314802000\end{array}$

$\begin{array}{lll}3.436291000 & 0.837489000 & 1.182896000\end{array}$

$\begin{array}{lll}-2.012511000 & 0.425882000 & -0.079144000\end{array}$

$\begin{array}{lll}-3.362328000 & 0.156959000 & 0.301621000\end{array}$

$\begin{array}{lll}-3.954180000 & 1.408644000 & 0.945053000\end{array}$

$\begin{array}{lll}-3.391347000 & 1.672619000 & 1.853991000\end{array}$

$\begin{array}{lll}-3.899486000 & 2.256845000 & 0.246303000\end{array}$

$\begin{array}{lll}-5.006336000 & 1.251399000 & 1.224530000\end{array}$

$\begin{array}{lll}-4.059854000 & 0.646729000 & -1.692452000\end{array}$

$\begin{array}{lll}-4.101192000 & -0.193825000 & -0.983018000\end{array}$

$\begin{array}{lll}-5.157200000 & -0.419071000 & -0.775857000\end{array}$

$\begin{array}{lll}-4.419077000 & -1.238697000 & 1.597240000\end{array}$

$\begin{array}{lll}-3.389690000 & -1.016678000 & 1.278807000\end{array}$

$\begin{array}{lll}-2.794513000 & -0.782036000 & 2.175343000\end{array}$

$\begin{array}{lll}-2.968727000 & -1.918583000 & 0.809133000\end{array}$

$\begin{array}{lll}-3.648898000 & -1.079258000 & -1.457120000\end{array}$

$\begin{array}{lll}-0.383783000 & 0.970154000 & 1.961614000\end{array}$

$\begin{array}{lll}1.613439000 & -0.382865000 & -0.214361000\end{array}$ 


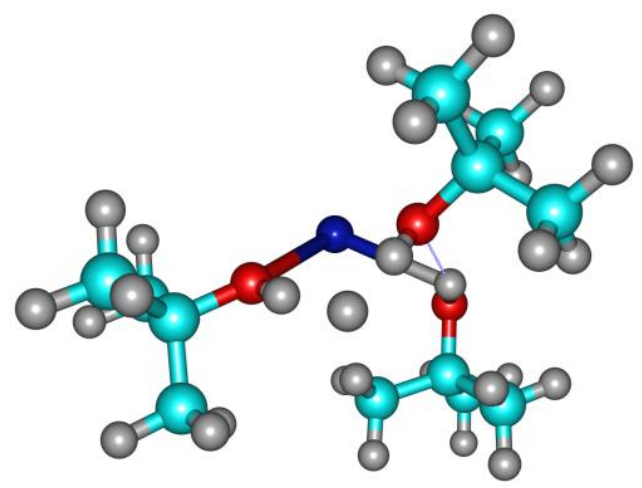

7

$G=-1300.342781$ (Temperature $=483.15$ pressure $=39.64$, def2SVP)

Thermal correction to $\mathrm{G}=\mathbf{0 . 3 0 6 8 4 4}$ (def2SVP)

$E=\mathbf{- 1 3 0 1 . 6 2 1 8 2 8}$ (def2QZVP)

$\begin{array}{lrrr}\text { 1 } & -1.456491000 & -0.730416000 & -0.844006000 \\ 19 & -0.300853000 & 0.002862000 & 1.876405000 \\ 6 & 1.405549000 & 2.415454000 & -0.246350000 \\ 8 & 1.720934000 & 1.366868000 & 0.675189000 \\ 6 & 2.115933000 & 2.143362000 & -1.569863000 \\ 1 & 1.751290000 & 1.200262000 & -2.008436000 \\ 1 & 1.927795000 & 2.951106000 & -2.292064000 \\ 1 & 3.201751000 & 2.062418000 & -1.409649000 \\ 6 & 1.901551000 & 3.707965000 & 0.384992000 \\ 1 & 1.681381000 & 4.568732000 & -0.262657000 \\ 1 & 1.412775000 & 3.871790000 & 1.357570000 \\ 1 & 2.988389000 & 3.658471000 & 0.548142000 \\ 6 & -0.108022000 & 2.456018000 & -0.460260000 \\ 1 & -0.628097000 & 2.701694000 & 0.481853000 \\ 1 & -0.387218000 & 3.220406000 & -1.200818000 \\ 1 & -0.469149000 & 1.481191000 & -0.825815000 \\ 1 & 0.447641000 & -1.151408000 & -0.933355000 \\ 8 & 1.036990000 & -1.138790000 & -0.090635000 \\ 6 & 2.089703000 & -2.098220000 & -0.195729000 \\ 6 & 1.483124000 & -3.490214000 & -0.356389000 \\ 1 & 0.833245000 & -3.725421000 & 0.500294000 \\ 1 & 0.875832000 & -3.538275000 & -1.272815000 \\ 1 & 2.269224000 & -4.257390000 & -0.418938000 \\ 1 & 2.371442000 & -1.753978000 & -2.320367000 \\ 6 & 2.968319000 & -1.747759000 & -1.395508000 \\ 1 & 3.790644000 & -2.470059000 & -1.507450000 \\ 1 & 3.747649000 & -2.685482000 & 1.083456000 \\ 6 & 2.889488000 & -1.997778000 & 1.098395000 \\ 1 & 2.260850000 & -2.258944000 & 1.965040000 \\ 1 & 3.269617000 & -0.972946000 & 1.232472000 \\ 1 & 3.402572000 & -0.744051000 & -1.269080000 \\ 8 & -1.956977000 & -0.491326000 & -0.004864000 \\ 6 & -3.332332000 & -0.281898000 & -0.307288000 \\ 6 & -3.929744000 & -1.582086000 & -0.843294000 \\ 1 & -3.418347000 & -1.880337000 & -1.771377000 \\ 1 & -3.802567000 & -2.389090000 & -0.105953000 \\ 1 & -5.002794000 & -1.469561000 & -1.058651000 \\ 1 & -3.887328000 & -0.679513000 & 1.752917000 \\ 6 & -4.001852000 & 0.118232000 & 1.002393000 \\ 1 & -5.076652000 & 0.299358000 & 0.855605000 \\ 1 & -4.520653000 & 1.008989000 & -1.601427000 \\ 6 & -3.465615000 & 0.836838000 & -1.340480000 \\ 1 & -2.919115000 & 0.573979000 & -2.259315000 \\ 1 & -3.048039000 & 1.775975000 & -0.946336000 \\ 1 & -3.552139000 & 1.044054000 & 1.396499000 \\ 1 & -0.486760000 & -1.048440000 & -1.981379000 \\ 1 & 1.635291000 & 0.509009000 & 0.203121000 \\ & & & \end{array}$

$\begin{array}{lll}1.635291000 & 0.509009000 & 0.203121000\end{array}$

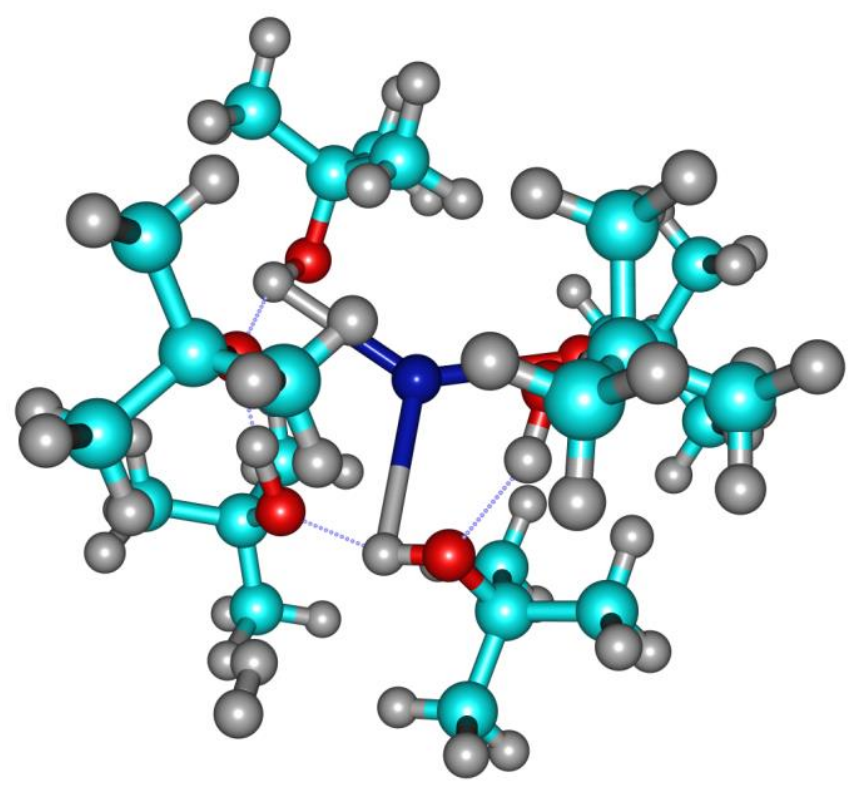

8

$G=-2000.257373$ (Temperature $=483.15$ pressure $=39.64$, def2SVP)

Thermal correction to $\mathrm{G}=\mathbf{0 . 6 4 7 8 9 7}$ (def2SVP)

$E=\mathbf{- 2 0 0 2 . 7 5 4 2 9 1}$ (def2QZVP)

190.100792000

$\begin{array}{llll}6 & 2.887818000 & -0.581779000 & 1.864702000\end{array}$

$\begin{array}{llll}8 & 2.602114000 & -0.561442000 & 0.493631000\end{array}$

$\begin{array}{llll}6 & 4.010305000 & 0.416232000 & 2.183936000\end{array}$

$\begin{array}{llll}1 & 3.720342000 & 1.427473000 & 1.861027000\end{array}$

$14.234743000 \quad 0.447027000 \quad 3.261813000$

$\begin{array}{llll}1 & 4.931095000 & 0.136292000 & 1.648023000\end{array}$

$\begin{array}{llll}6 & 3.338287000 & -1.983601000 & 2.286095000\end{array}$

$\begin{array}{llll}1 & 3.626712000 & -2.016130000 & 3.348473000\end{array}$

$\begin{array}{llll}1 & 2.529270000 & -2.714654000 & 2.131834000\end{array}$

$\begin{array}{llll}1 & 4.204774000 & -2.293686000 & 1.681061000\end{array}$

$\begin{array}{llll}6 & 1.626119000 & -0.195865000 & 2.649852000\end{array}$

$\begin{array}{llll}1 & 0.818333000 & -0.912457000 & 2.428458000\end{array}$

$\begin{array}{llll}1 & 1.792591000 & -0.188386000 & 3.739218000\end{array}$

$\begin{array}{llll}1 & 1.280707000 & 0.808314000 & 2.348410000\end{array}$

$8 \quad 1.806947000 \quad-2.155104000-1.388981000$

$\begin{array}{llll}1 & 2.237837000 & -1.729028000 & -0.586750000\end{array}$

$1 \quad-0.106749000 \quad-3.358487000 \quad-2.692570000$

$\begin{array}{llll}6 & 0.721835000 & -4.006495000 & -2.367326000\end{array}$

$1 \quad 1.472626000 \quad-4.035491000 \quad-3.171068000$

$\begin{array}{llll}1 & 0.329433000 & -5.022668000 & -2.215849000\end{array}$

$1 \quad 3.290864000 \quad-4.336069000 \quad-1.452440000$

$\begin{array}{llll}6 & 1.344899000 & -3.463593000 & -1.087179000\end{array}$

$\begin{array}{llll}1 & -0.621674000 & -2.892758000 & -0.323877000\end{array}$

$\begin{array}{llll}6 & 0.292369000 & -3.401263000 & 0.026601000\end{array}$

$\begin{array}{lll}0.691960000 & -2.860113000 & 0.899758000\end{array}$

$\begin{array}{lll}2.983741000 & -3.938635000 & 0.257294000\end{array}$

$\begin{array}{lll}-0.003386000 & -4.407991000 & 0.357283000\end{array}$

$\begin{array}{lll}2.212920000 & -5.372189000 & -0.472943000\end{array}$

$2.526180000 \quad-4.334496000 \quad-0.661328000$

$\begin{array}{lll}2.241421000 & 3.455617000 & 1.529826000\end{array}$

$\begin{array}{lll}2.146052000 & 1.705097000 & -0.327687000\end{array}$

$\begin{array}{lll}2.935214000 & 1.972994000 & -1.477848000\end{array}$

$\begin{array}{lll}2.793969000 & 3.452600000 & -1.821412000\end{array}$

$\begin{array}{lll}1.751571000 & 3.702019000 & -2.070234000\end{array}$

$\begin{array}{lll}3.105429000 & 4.072648000 & -0.966854000\end{array}$

$3.420890000 \quad 3.716030000 \quad-2.686491000$

$\begin{array}{lll}4.766454000 & 2.276155000 & -0.346863000\end{array}$

$\begin{array}{lll}4.400300000 & 1.649071000 & -1.174556000\end{array}$

$\begin{array}{lll}5.036148000 & 1.828596000 & -2.054542000\end{array}$

$3.037801000 \quad 1.292314000 \quad-3.556481000$

$\begin{array}{lll}2.450235000 & 1.105160000 & -2.644736000\end{array}$

$\begin{array}{lll}1.395150000 & 1.326059000 & -2.878187000\end{array}$

$\begin{array}{lll}2.544504000 & 0.035557000 & -2.396317000\end{array}$ 


$\begin{array}{rrrr}1 & 4.494181000 & 0.594039000 & -0.878510000 \\ 8 & -0.328616000 & 2.246416000 & 0.642889000 \\ 6 & -1.143692000 & 3.185885000 & -0.065045000 \\ 6 & -1.190092000 & 2.804066000 & -1.544250000 \\ 1 & -0.175325000 & 2.791225000 & -1.970288000 \\ 1 & -1.643185000 & 1.807219000 & -1.664795000 \\ 1 & -1.791315000 & 3.523112000 & -2.120508000 \\ 1 & -2.925654000 & 2.061149000 & 0.412585000 \\ 6 & -2.534776000 & 3.081556000 & 0.545001000 \\ 1 & -3.228234000 & 3.783922000 & 0.060340000 \\ 1 & -1.186248000 & 5.340109000 & -0.381957000 \\ 6 & -0.565585000 & 4.584960000 & 0.122352000 \\ 1 & 0.451243000 & 4.637889000 & -0.296942000 \\ 1 & -0.511722000 & 4.831325000 & 1.193149000 \\ 1 & -2.498761000 & 3.311397000 & 1.621308000 \\ 1 & -1.361657000 & 0.867465000 & 1.591081000 \\ 8 & -1.664273000 & -0.057120000 & 1.519321000 \\ 6 & -2.427917000 & -0.452458000 & 2.665749000 \\ 6 & -3.845160000 & 0.100051000 & 2.534117000 \\ 1 & -3.836727000 & 1.200470000 & 2.540975000 \\ 1 & -4.299346000 & -0.239770000 & 1.589838000 \\ 1 & -4.479490000 & -0.243312000 & 3.364472000 \\ 1 & -2.873747000 & -2.338733000 & 1.698850000 \\ 6 & -2.439568000 & -1.974966000 & 2.643356000 \\ 1 & -3.037760000 & -2.372426000 & 3.475558000 \\ 1 & -2.358000000 & -0.183838000 & 4.817597000 \\ 6 & -1.762252000 & 0.076709000 & 3.930802000 \\ 1 & -1.676103000 & 1.174509000 & 3.888000000 \\ 1 & -0.755765000 & -0.347629000 & 4.051001000 \\ 1 & -1.415029000 & -2.367558000 & 2.729075000 \\ 1 & -2.596951000 & -0.379408000 & -0.144819000 \\ 8 & -2.454813000 & -0.520591000 & -1.099912000 \\ 6 & -4.587251000 & 0.501366000 & -1.587360000 \\ 1 & -4.810216000 & 0.659954000 & -0.520233000 \\ 1 & -4.089205000 & 1.402717000 & -1.975739000 \\ 1 & -5.542576000 & 0.375921000 & -2.118026000 \\ 1 & -2.826730000 & -0.025958000 & -3.630991000 \\ 6 & -3.345320000 & -0.915749000 & -3.242962000 \\ 1 & -4.252624000 & -1.078120000 & -3.841722000 \\ 1 & -5.309844000 & -2.190254000 & -1.742672000 \\ 6 & -4.372581000 & -1.972387000 & -1.210002000 \\ 1 & -4.612411000 & -1.831083000 & -0.144024000 \\ 1 & -3.705768000 & -2.842780000 & -1.304667000 \\ 1 & -2.684947000 & -1.786637000 & -3.368917000 \\ 6 & -3.697063000 & -0.725038000 & -1.774011000 \\ 1 & 2.343580000 & 3.939524000 & 2.102773000 \\ 1 & 2.405685000 & 0.718602000 & 0.059382000\end{array}$

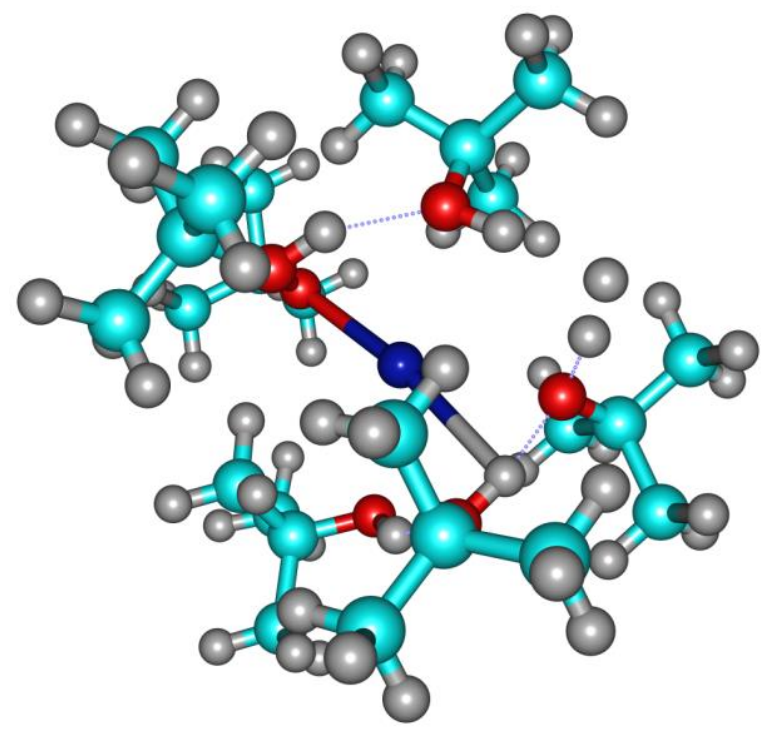

$\mathrm{TS}_{5}$

$G=-2000.233653$ (Temperature $=483.15$ pressure $=39.64$, def2SVP) $v=1300 \mathrm{~cm}^{-1}$

Thermal correction to $\mathrm{G}=\mathbf{0 . 6 4 8 2 3 1}$ (def2SVP)

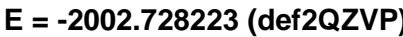

$\begin{array}{lll}-0.501653000 & 2.664785000 & -0.989025000\end{array}$

$\begin{array}{llll}19 & -0.228752000 & 0.067471000 & 0.500680000\end{array}$

$\begin{array}{llll}6 & -2.915817000 & -0.904238000 & -1.970896000\end{array}$

$\begin{array}{llll}8 & -2.748286000 & -0.952248000 & -0.556017000\end{array}$

$\begin{array}{llll}6 & -4.145681000 & -0.057870000 & -2.296655000\end{array}$

$\begin{array}{llll}1 & -4.007224000 & 0.968178000 & -1.921859000\end{array}$

$\begin{array}{llll}1 & -4.316043000 & -0.009048000 & -3.382341000\end{array}$

$\begin{array}{llll}1 & -5.040483000 & -0.486934000 & -1.820439000\end{array}$

$6 \quad-3.105735000 \quad-2.338148000 \quad-2.446817000$

$\begin{array}{lll}-3.278307000 & -2.370551000 & -3.532395000\end{array}$

$\begin{array}{lll}-2.212344000 & -2.941377000 & -2.222716000\end{array}$

$\begin{array}{lll}-3.969996000 & -2.793850000 & -1.940361000\end{array}$

$\begin{array}{lll}-1.666223000 & -0.289582000 & -2.604227000\end{array}$

$\begin{array}{lll}-0.774176000 & -0.885200000 & -2.351882000\end{array}$

$\begin{array}{lll}-1.753930000 & -0.251156000 & -3.700684000\end{array}$

$\begin{array}{lll}-1.517167000 & 0.742665000 & -2.243748000\end{array}$

$\begin{array}{lll}-1.368570000 & -2.236976000 & 1.427644000\end{array}$

$\begin{array}{lll}-1.985492000 & -2.023980000 & 0.696520000\end{array}$

$\begin{array}{lll}0.852120000 & -2.920131000 & 2.578218000\end{array}$

$\begin{array}{lll}0.200321000 & -3.763497000 & 2.302254000\end{array}$

$\begin{array}{lll}-0.459835000 & -3.983936000 & 3.154275000\end{array}$

$\begin{array}{lll}0.831008000 & -4.642980000 & 2.107254000\end{array}$

$\begin{array}{lll}-2.277555000 & -4.715016000 & 1.558664000\end{array}$

$\begin{array}{lll}-0.623727000 & -3.403201000 & 1.074826000\end{array}$

$\begin{array}{lll}1.050132000 & -2.335976000 & 0.175588000\end{array}$

$\begin{array}{lll}0.292611000 & -3.084075000 & -0.109314000\end{array}$

$\begin{array}{lll}-0.298744000 & -2.692379000 & -0.953596000\end{array}$

$-2.198645000 \quad-4.241534000 \quad-0.159869000$

$\begin{array}{lll}0.825075000 & -3.982507000 & -0.455802000\end{array}$

$\begin{array}{lll}-1.059143000 & -5.455574000 & 0.480392000\end{array}$

$\begin{array}{lll}-1.595053000 & -4.524798000 & 0.717078000\end{array}$

$\begin{array}{lll}-2.331345000 & 2.334714000 & -0.375477000\end{array}$

$\begin{array}{lll}-2.510600000 & 1.412773000 & 0.427957000\end{array}$

$\begin{array}{lll}-3.313744000 & 1.669368000 & 1.563025000\end{array}$

$\begin{array}{lll}-3.446048000 & 3.177787000 & 1.788458000\end{array}$

$\begin{array}{lll}-2.457581000 & 3.642930000 & 1.925529000\end{array}$

$\begin{array}{lll}-3.932632000 & 3.658055000 & 0.926242000\end{array}$

$\begin{array}{lll}-4.049886000 & 3.386778000 & 2.684553000\end{array}$

$\begin{array}{lll}-5.191155000 & 1.522965000 & 0.479435000\end{array}$

$\begin{array}{lll}-4.703941000 & 1.059799000 & 1.351616000\end{array}$

$\begin{array}{lll}-5.349895000 & 1.211858000 & 2.230056000\end{array}$

$\begin{array}{lll}-3.232157000 & 1.162321000 & 3.695275000\end{array}$

$\begin{array}{lll}-2.643772000 & 1.015809000 & 2.776309000\end{array}$

$\begin{array}{lll}-1.644495000 & 1.452283000 & 2.942311000\end{array}$

$\begin{array}{lll}-2.532923000 & -0.069432000 & 2.615092000\end{array}$

$\begin{array}{lll}-4.620681000 & -0.021867000 & 1.163937000\end{array}$

$\begin{array}{lll}0.447651000 & 2.403854000 & -0.895966000\end{array}$

$\begin{array}{lll}1.128077000 & 3.370810000 & -0.080873000\end{array}$

$\begin{array}{lll}0.754974000 & 3.138767000 & 1.382957000\end{array}$

$\begin{array}{lll}-0.340392000 & 3.135645000 & 1.494929000\end{array}$

$\begin{array}{lll}1.160586000 & 2.176745000 & 1.736553000\end{array}$

$\begin{array}{lll}1.166860000 & 3.929400000 & 2.027242000\end{array}$

$\begin{array}{lll}2.867592000 & 2.089283000 & -0.048347000\end{array}$

$\begin{array}{lll}2.615963000 & 3.131205000 & -0.297347000\end{array}$

$\begin{array}{lll}3.213064000 & 3.793774000 & 0.346150000\end{array}$

$\begin{array}{lll}1.301521000 & 5.533510000 & 0.022480000\end{array}$

$\begin{array}{lll}0.729446000 & 4.773136000 & -0.528758000\end{array}$

$\begin{array}{lll}-0.342421000 & 4.947520000 & -0.349099000\end{array}$

$\begin{array}{lll}0.926367000 & 4.896276000 & -1.604220000\end{array}$

$\begin{array}{lll}2.887712000 & 3.324462000 & -1.346370000\end{array}$

$\begin{array}{lll}1.387442000 & 0.969760000 & -1.513432000\end{array}$

$\begin{array}{lll}1.673402000 & 0.033316000 & -1.489228000\end{array}$

$\begin{array}{lll}2.405324000 & -0.285192000 & -2.679949000\end{array}$

$\begin{array}{lll}3.863213000 & 0.123935000 & -2.484685000\end{array}$

$\begin{array}{lll}3.941318000 & 1.207418000 & -2.307753000\end{array}$

$\begin{array}{lll}4.292693000 & -0.403971000 & -1.618636000\end{array}$

$\begin{array}{lll}4.465185000 & -0.124681000 & -3.371105000\end{array}$

$\begin{array}{lll}2.698069000 & -2.318362000 & -1.987862000\end{array}$

$\begin{array}{lll}2.291735000 & -1.791932000 & -2.865400000\end{array}$

$2.854322000 \quad-2.116176000 \quad-3.752644000$

$\begin{array}{lll}2.340722000 & 0.259164000 & -4.783146000\end{array}$

$\begin{array}{lll}1.785585000 & 0.461912000 & -3.856002000\end{array}$ 


$\begin{array}{rrr}1.805502000 & 1.548386000 & -3.673736000 \\ 0.739511000 & 0.155585000 & -4.001740000 \\ 1.238676000 & -2.085707000 & -2.992329000 \\ 2.473061000 & -0.234397000 & 0.230513000 \\ 2.295392000 & -0.235224000 & 1.191287000 \\ 4.568699000 & 0.430651000 & 1.645732000 \\ 4.808148000 & 0.467238000 & 0.570894000 \\ 4.241345000 & 1.432175000 & 1.962824000 \\ 5.488519000 & 0.175646000 & 2.192413000 \\ 2.728141000 & 0.408577000 & 3.683607000 \\ 3.079344000 & -0.590750000 & 3.385559000 \\ 3.937471000 & -0.860416000 & 4.017391000 \\ 4.817356000 & -2.308178000 & 2.025967000 \\ 3.918579000 & -1.990292000 & 1.477436000 \\ 4.155904000 & -1.997280000 & 0.401090000 \\ 3.119542000 & -2.724507000 & 1.661531000 \\ 2.270314000 & -1.313248000 & 3.569979000 \\ 3.472254000 & -0.597228000 & 1.914820000 \\ -2.093466000 & 3.037025000 & -1.072521000 \\ -2.759427000 & -0.004922000 & -0.196998000\end{array}$

$-1.132803000$

1.941232000

0.375120000

$-1.232355000$

$-0.546492000$

0.046512000

1.858264000

$-1.160113000$

0.649390000

1.255593000

2.492630000

2.537304000

3.570839000

3.363130000

2.385520000

3.399016000

4.145948000

4.989754000

4.926946000

5.748075000

4.236836000

3.444887000

2.471358000

3.525683000

5.064779000

$-0.224798000$

$-0.869045000$

$-0.322344000$

0.769508000

$-0.557287000$

$-0.765228000$

$-2.532656000$

$-2.357745000$

$-2.912808000$

$-1.147604000$

$-0.609715000$

0.465000000

$-0.949674000$

$-2.754863000$

$-1.297148000$

$-1.685135000$

$-2.435383000$

$-3.892490000$

$-3.973654000$

$-4.307482000$

$-4.504809000$

$-2.698895000$

$-2.322212000$

$-2.913314000$

$-2.397136000$

$-1.841575000$

$-1.886342000$

$-0.788917000$

$-1.273876000$

$-2.500198000$

$-2.348859000$

$-4.596698000$

$-4.786947000$

$-4.231181000$

$-5.551419000$

$-2.826001000$

$-3.230945000$

$-4.127357000$

$-5.014111000$

$-4.078857000$

$-4.275471000$

$-3.330908000$

$-2.478701000$

$-3.570171000$

2.221614000

2.787302000
$-4.700459000$

$-4.961452000$

$-3.514385000$

$-2.281928000$

$-3.075694000$

$-2.697749000$

$-4.399604000$

$-3.914319000$

$-5.560090000$

$-4.682159000$

2.019563000

1.216371000

1.357524000

2.657832000

2.652352000

3.519766000

2.787974000

2.194682000

1.356204000

1.454218000

0.159684000

0.151797000

0.159844000

$-0.784121000$

0.416053000

2.437253000

3.503429000

3.558809000

3.690361000

2.628830000

4.395354000

2.192081000

3.181611000

3.930182000

5.647835000

4.819663000

5.054469000

4.744201000

3.181618000

1.141384000

0.240489000

0.054810000

0.424344000

1.473478000

$-0.218652000$

0.291676000

$-2.048326000$

$-1.419050000$

$-1.639087000$

0.823347000

0.939342000

1.999304000

0.676309000

$-1.690526000$

$-0.107360000$

$-0.223073000$

0.533141000

0.689399000

1.478070000

0.278274000

0.213952000

$-0.729487000$

$-0.994123000$

$-2.207847000$

$-1.896579000$

$-1.790234000$

$-2.691691000$

$-1.519478000$

$-0.578486000$

3.001404000

$-0.211825000$
$-1.864698000$

$-1.223697000$

$-0.845625000$

$-0.043111000$

0.294606000

1.143735000

0.467950000

0.656693000

$-0.143075000$

$-0.409993000$

$-0.069594000$

$-0.710020000$

$-1.686566000$

$-2.458465000$

$-2.963634000$

$-1.774575000$

$-3.220584000$

$-0.272426000$

$-0.983180000$

$-1.708828000$

$-3.374745000$

$-2.611527000$

$-3.127169000$

$-2.036984000$

$-0.427417000$

0.688567000

$-0.018242000$

$-1.444078000$

$-1.425008000$

$-1.988543000$

$-2.005021000$

$-0.473838000$

$-0.026033000$

$-0.610582000$

0.226604000

0.711315000

0.714085000

1.755279000

1.000917000

1.370734000

1.394200000

2.602650000

2.335542000

2.013788000

1.543415000

3.239690000

2.148953000

2.970061000

3.870809000

4.634835000

3.693256000

3.395576000

3.873961000

3.165771000

$-0.298075000$

$-1.256924000$

$-1.703467000$

$-0.629639000$

$-2.132446000$

$-2.186619000$

$-3.780548000$

$-3.384704000$

$-3.963220000$

$-1.817306000$

$-1.329302000$

$-0.250013000$

$-1.469734000$

$-3.527913000$

$-1.908555000$

0.893175000

0.221988000 


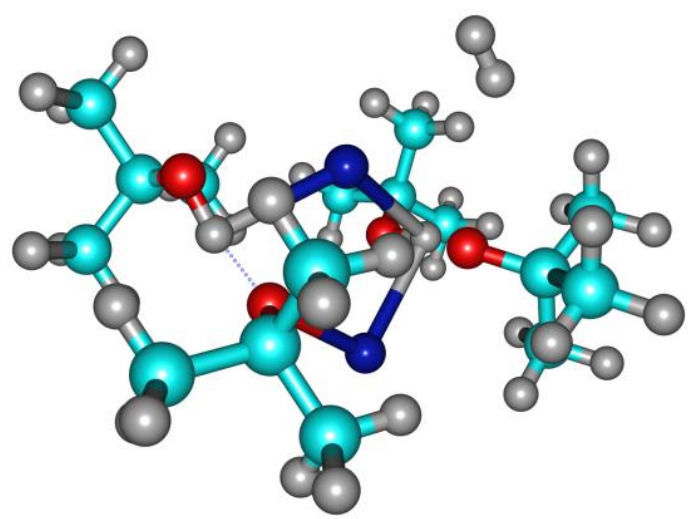

10

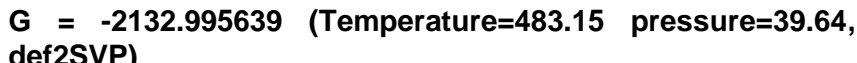

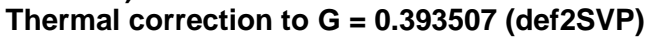

$E=-\mathbf{2 1 3 4 . 7 4 1 3 9 1}$ (def2QZVP)

\begin{tabular}{|c|c|c|c|}
\hline & 2.954789000 & 0.327685000 & -0.910790000 \\
\hline & 4.350542000 & 0.926615000 & 0.959533000 \\
\hline & 4.789253000 & 1.761878000 & 1.526306000 \\
\hline & 3.519895000 & 1.427382000 & -0.225436000 \\
\hline & 1.716706000 & 1.799232000 & 0.940755000 \\
\hline & 2.400763000 & 2.349627000 & 0.273440000 \\
\hline & 2.794690000 & 3.212562000 & 0.831846000 \\
\hline & 1.823417000 & 2.743457000 & -0.580337000 \\
\hline 1 & 5.189528000 & 1.498897000 & -1.598680000 \\
\hline 1 & 4.897663000 & 3.039736000 & -0.740929000 \\
\hline & 4.410293000 & 2.173971000 & -1.213756000 \\
\hline & 3.815986000 & 2.533594000 & -2.068129000 \\
\hline 1 & 5.168423000 & 0.281285000 & 0.603558000 \\
\hline 1 & 3.718560000 & 0.334906000 & 1.639731000 \\
\hline 1 & 2.432682000 & 2033000 & 9000 \\
\hline 8 & -1.931169000 & -0.684968000 & -0.448104000 \\
\hline & -3.650693000 & -1.634384000 & 0.948020000 \\
\hline 1 & -3.769572000 & -0.664662000 & 1.460807000 \\
\hline 6 & -3.111325000 & -1.420198000 & -0.478805000 \\
\hline 1 & -2.073554000 & -3.330377000 & -0.541902000 \\
\hline & -2.844911000 & -2.791813000 & -1.115380000 \\
\hline 1 & -3.750090000 & -3.418934000 & -1.149246000 \\
\hline 1 & -2.475413000 & -2.664260000 & -2.145666000 \\
\hline 1 & -3.808705000 & -0.510522000 & -2.326922000 \\
\hline 1 & -4.421103000 & 0.282549000 & -0.853068000 \\
\hline 6 & -4.182029000 & -0.691909000 & -1.306484000 \\
\hline 1 & -5.116962000 & -1.269733000 & -1.380981000 \\
\hline 1 & -2.960519000 & -2.267536000 & 1.533445000 \\
\hline 1 & -4.628608000 & -2.140807000 & 0.955566000 \\
\hline 1 & -1.80594 & 1000 & 0.1 \\
\hline 19 & -0.5 & -0.7 & 44000 \\
\hline 8 & -1.333829000 & 1.423701000 & 0.635903000 \\
\hline 6 & -1.136306000 & 3.754548000 & 1.035852000 \\
\hline 1 & -1.583963000 & 4.746090000 & 0.872301000 \\
\hline 6 & -1.975156000 & 2.660077000 & 0.383594000 \\
\hline 1 & -3.994105000 & 1.861946000 & 0.530649000 \\
\hline 6 & -3.377725000 & 2.646239000 & 0.995160000 \\
\hline 1 & -3.3 & & \\
\hline 1 & -3.888237 & 3.610546000 & 0.852288000 \\
\hline 1 & -1.053276000 & 836000 & -1.565978000 \\
\hline 1 & -2.608456000 & 3.814803000 & -1.365808000 \\
\hline 6 & -2.061882000 & 2.889804000 & -1.128788000 \\
\hline 1 & -2.584499000 & 2.050256000 & -1.615102000 \\
\hline 1 & -0.118326000 & 3.760042000 & 0.617946000 \\
\hline 1 & -1.062566000 & 3.582707000 & 2.120744000 \\
\hline 19 & 0.272689000 & 0.294821000 & -1.346810000 \\
\hline 8 & 1.537513000 & -1.113732000 & 0.549188000 \\
\hline 6 & 3.317316000 & -2.729966000 & 0.505213000 \\
\hline 1 & 3.933618000 & -2.053447000 & -0.107057000 \\
\hline 6 & 1.830040000 & -2.443868000 & 0.243766000 \\
\hline 1 & 2.117513000 & -2.071718000 & -1.886059000 \\
\hline 6 & 1.518617000 & -2.733061000 & -1.237196000 \\
\hline & & & \\
\hline
\end{tabular}

$\begin{array}{rrrr}1 & 0.446069000 & -2.563304000 & -1.436908000 \\ 1 & 1.216287000 & -4.445927000 & 0.925756000 \\ 1 & -0.093987000 & -3.254017000 & 0.887346000 \\ 6 & 0.979540000 & -3.386510000 & 1.110250000 \\ 1 & 1.157209000 & -3.187085000 & 2.181009000 \\ 1 & 3.593869000 & -3.768876000 & 0.265020000 \\ 1 & 3.554640000 & -2.543629000 & 1.565008000 \\ 1 & -1.177529000 & -0.001034000 & -3.931465000 \\ 1 & -1.630175000 & -0.226363000 & -3.368481000\end{array}$

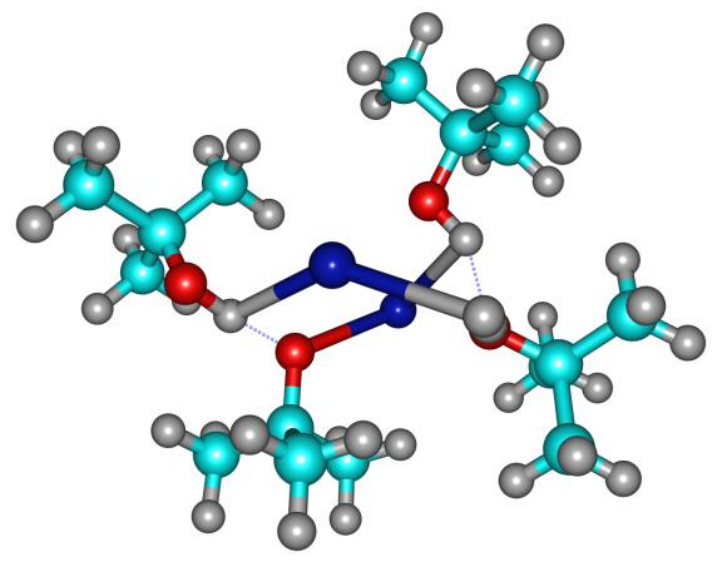

$\mathrm{TS}_{6}$

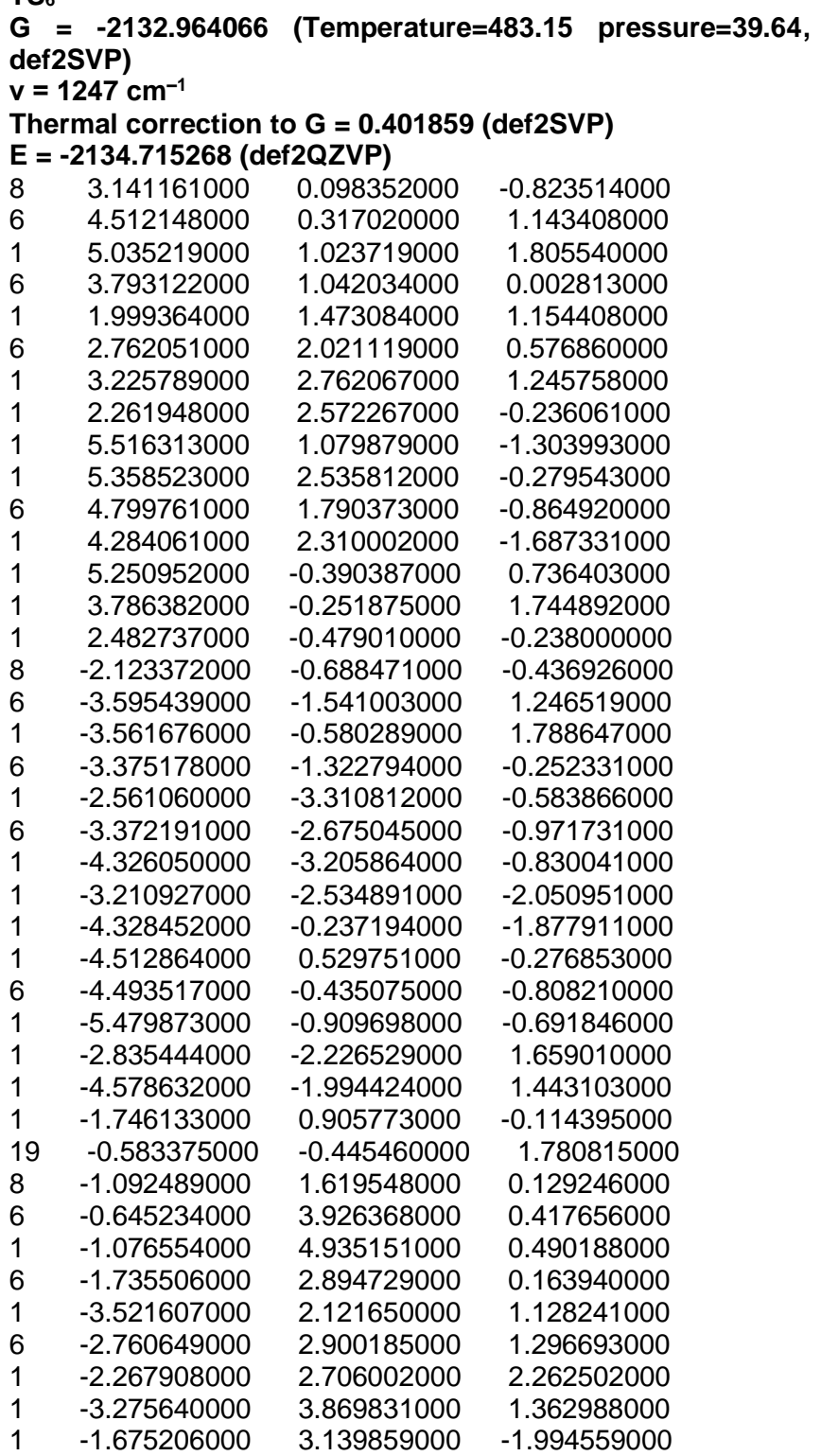




$\begin{array}{lrrr}1 & -2.933155000 & 4.114583000 & -1.188012000 \\ 6 & -2.416349000 & 3.143636000 & -1.180056000 \\ 1 & -3.157503000 & 2.355347000 & -1.386358000 \\ 1 & 0.086682000 & 3.923174000 & -0.404332000 \\ 1 & -0.116540000 & 3.706721000 & 1.357873000 \\ 19 & 0.579115000 & 0.398416000 & -1.595948000 \\ 8 & 1.395961000 & -1.192063000 & 0.399836000 \\ 6 & 2.855552000 & -3.103327000 & 0.346249000 \\ 1 & 3.593081000 & -2.537007000 & -0.243668000 \\ 6 & 1.447940000 & -2.550394000 & 0.077269000 \\ 1 & 1.819511000 & -2.229127000 & -2.051263000 \\ 6 & 1.099672000 & -2.763765000 & -1.407287000 \\ 1 & 1.128866000 & -3.826339000 & -1.695260000 \\ 1 & 0.080053000 & -2.392830000 & -1.612615000 \\ 1 & 0.454129000 & -4.410137000 & 0.715857000 \\ 1 & -0.594188000 & -2.979142000 & 0.700594000 \\ 6 & 0.428702000 & -3.329132000 & 0.923889000 \\ 1 & 0.637404000 & -3.192506000 & 1.999203000 \\ 1 & 2.943466000 & -4.169662000 & 0.084583000 \\ 1 & 3.108817000 & -2.986387000 & 1.412261000 \\ 1 & -1.695129000 & -0.369606000 & -2.639455000 \\ 1 & -1.917206000 & -0.545604000 & -1.631917000\end{array}$

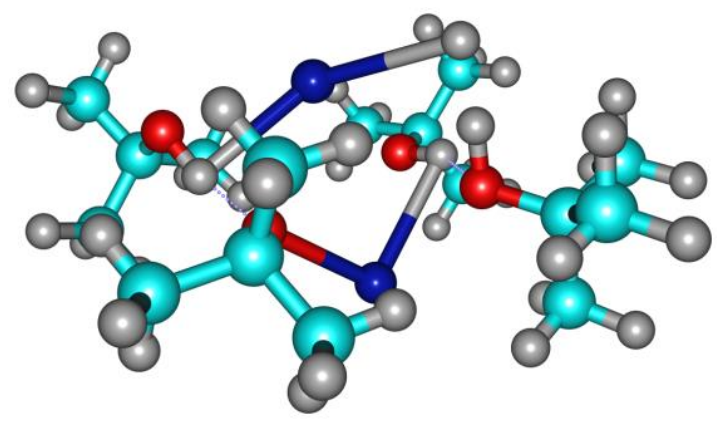

-0.711551000
-1.552595000
-3.369521000
-2.545173000
-2.045788000
-2.979593000
-1.520736000
-2.669273000
-2.239374000
-3.051439000
0.339869000
0.158063000
0.664818000
1.307698000
2.685585000
3.461955000
1.310373000
1.754387000
0.993478000
0.982514000
-0.000372000
0.217254000
-0.762514000
0.236992000
0.428879000
2.734051000
2.915923000
-1.666535000
-2.011364000

$4.959521000 \quad 0.495275000$ $2.996167000 \quad 0.141956000$ $2.342340000 \quad 1.135991000$ $3.054786000 \quad 1.300601000$ $2.802834000 \quad 2.249572000$ $4.060253000 \quad 1.399213000$ $3.282767000-2.010943000$ $4.344323000-1.151264000$ $3.332442000-1.178426000$ $2.616586000-1.382932000$ $3.873708000-0.448228000$ $3.634682000 \quad 1.314219000$ $0.425017000-1.676209000$ $\begin{array}{ll}-1.216422000 & 0.352194000\end{array}$ $-3.189484000 \quad 0.362195000$ $-2.661839000 \quad-0.213374000$ $-2.580712000 \quad 0.048835000$ $-2.305262000 \quad-2.070995000$ $-2.798448000-1.441296000$ $-3.865204000-1.715079000$ $-2.382013000-1.681304000$ $-4.386956000 \quad 0.677420000$ $-2.907538000 \quad 0.629147000$ $-3.304389000 \quad 0.877758000$ $-3.171916000 \quad 1.957023000$ $-4.261551000 \quad 0.114055000$ $-3.071780000 \quad 1.433398000$ $-0.311176000-2.690746000$ $-0.604686000-1.390331000$

11

$G=-2132.964461$ (Temperature $=483.15$ pressure=39.64, def2SVP)

Thermal correction to $\mathrm{G}=\mathbf{0 . 4 0 3 7 8 4}$ (def2SVP)

$E=-2134.717963$ (def2QZVP)

$\begin{array}{lccc}8 & 3.175995000 & -0.040102000 & -0.801526000 \\ 6 & 4.445580000 & 0.099948000 & 1.241624000 \\ 1 & 4.985201000 & 0.775255000 & 1.923004000 \\ 6 & 3.854429000 & 0.860149000 & 0.051269000 \\ 1 & 2.032976000 & 1.453118000 & 1.080851000 \\ 6 & 2.874478000 & 1.928791000 & 0.550083000 \\ 1 & 3.353501000 & 2.638564000 & 1.241723000 \\ 1 & 2.475826000 & 2.507443000 & -0.299043000 \\ 1 & 5.650138000 & 0.736745000 & -1.147754000 \\ 1 & 5.549034000 & 2.219663000 & -0.155336000 \\ 6 & 4.968144000 & 1.510090000 & -0.763300000 \\ 1 & 4.546080000 & 2.053288000 & -1.623004000 \\ 1 & 5.147867000 & -0.670726000 & 0.888457000 \\ 1 & 3.644224000 & -0.399101000 & 1.808097000 \\ 1 & 2.457311000 & -0.571269000 & -0.243708000 \\ 8 & -2.216385000 & -0.717173000 & -0.376272000 \\ 6 & -3.737876000 & -1.467268000 & 1.283882000 \\ 1 & -3.644315000 & -0.511393000 & 1.826604000 \\ 6 & -3.526277000 & -1.260998000 & -0.212996000 \\ 1 & -2.851096000 & -3.293668000 & -0.567395000 \\ 6 & -3.611297000 & -2.596012000 & -0.950824000 \\ 1 & -4.602874000 & -3.054813000 & -0.821370000 \\ 1 & -3.432722000 & -2.448904000 & -2.026436000 \\ 1 & -4.368009000 & -0.083888000 & -1.830193000 \\ 1 & -4.489396000 & 0.684746000 & -0.222466000 \\ 6 & -4.554885000 & -0.273119000 & -0.762612000 \\ 1 & -5.577807000 & -0.662714000 & -0.650881000 \\ 1 & -3.012798000 & -2.194627000 & 1.685749000 \\ 1 & -4.744187000 & -1.862179000 & 1.485189000 \\ 1 & -1.741746000 & 1.047275000 & -0.182876000 \\ 19 & -0.609233000 & -0.387569000 & 1.763441000 \\ 8 & -1.020729000 & 1.667237000 & 0.060812000 \\ 6 & -0.369944000 & 3.919765000 & 0.391519000\end{array}$

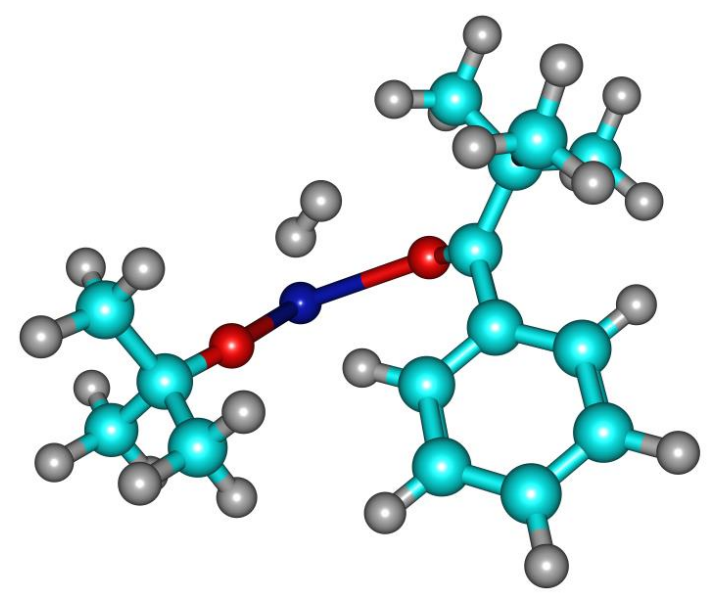

I

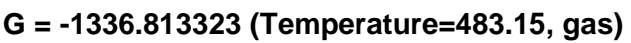

$E=-1337.06566494$ (gas)

$\begin{array}{llll}6 & -1.749833000 & -0.583711000 & -0.286966000\end{array}$

$\begin{array}{lllll}8 & -1.039461000 & -1.280911000 & -0.984726000\end{array}$

$\begin{array}{lllll}19 & 1.370313000 & -1.879591000 & -1.626924000\end{array}$

$\begin{array}{llll}8 & 2.488279000 & -0.546192000 & -0.121601000\end{array}$

$\begin{array}{llll}1 & 0.760665000 & -0.847070000 & 1.474473000\end{array}$

$\begin{array}{llll}1 & 0.182790000 & -0.926729000 & 1.941636000\end{array}$

$\begin{array}{llll}6 & -1.537794000 & 0.895477000 & -0.295530000\end{array}$

$\begin{array}{llll}6 & -2.829519000 & -1.243829000 & 0.579994000\end{array}$

$\begin{array}{llll}6 & 3.528761000 & 0.056422000 & 0.541887000\end{array}$

$\begin{array}{llll}6 & -2.596522000 & 1.802960000 & -0.314652000\end{array}$

$\begin{array}{llll}6 & -0.223440000 & 1.365744000 & -0.326195000\end{array}$

$\begin{array}{llll}6 & 0.013524000 & 2.732010000 & -0.363296000\end{array}$

$\begin{array}{llll}6 & -2.349856000 & 3.163829000 & -0.384500000\end{array}$

$\begin{array}{llll}6 & -1.042827000 & 3.630051000 & -0.401526000\end{array}$

$\begin{array}{llll}1 & -3.617846000 & 1.455834000 & -0.299572000\end{array}$

$\begin{array}{llll}1 & -3.176791000 & 3.858144000 & -0.420318000\end{array}$

$1 \quad-0.849399000 \quad 4.692655000 \quad-0.440244000$

$\begin{array}{llll}1 & 1.032695000 & 3.089474000 & -0.356763000\end{array}$

$1 \quad 0.621116000 \quad 0.678631000 \quad-0.282612000$

$\begin{array}{llll}6 & -3.069257000 & -0.484268000 & 1.887342000\end{array}$

$\begin{array}{llll}6 & -4.123909000 & -1.327480000 & -0.247433000\end{array}$

$\begin{array}{llll}6 & -2.378841000 & -2.670260000 & 0.905594000\end{array}$ 


$\begin{array}{rrrr}6 & 3.215422000 & 1.540137000 & 0.797623000 \\ 6 & 3.782827000 & -0.631236000 & 1.893873000 \\ 6 & 4.815786000 & -0.029132000 & -0.296064000 \\ 1 & -3.506540000 & 0.497243000 & 1.728931000 \\ 1 & -1.442241000 & -2.666695000 & 1.460872000 \\ 1 & -3.949395000 & -1.865519000 & -1.178123000 \\ 1 & 3.991330000 & -1.688635000 & 1.725937000 \\ 1 & 2.294011000 & 1.624144000 & 1.376952000 \\ 1 & 4.650854000 & 0.452820000 & -1.260959000 \\ 1 & 5.669478000 & 0.448133000 & 0.189768000 \\ 1 & 5.061756000 & -1.077430000 & -0.473901000 \\ 1 & 4.624040000 & -0.191672000 & 2.433728000 \\ 1 & 2.893805000 & -0.556315000 & 2.519934000 \\ 1 & 4.013660000 & 2.052524000 & 1.338196000 \\ 1 & 3.068603000 & 2.042576000 & -0.160413000 \\ 1 & -3.751130000 & -1.060348000 & 2.512183000 \\ 1 & -2.138673000 & -0.350610000 & 2.438020000 \\ 1 & -3.139605000 & -3.155381000 & 1.515562000 \\ 1 & -2.236977000 & -3.253384000 & -0.000387000 \\ 1 & -4.873220000 & -1.872572000 & 0.326456000 \\ 1 & -4.536604000 & -0.351040000 & -0.488083000\end{array}$

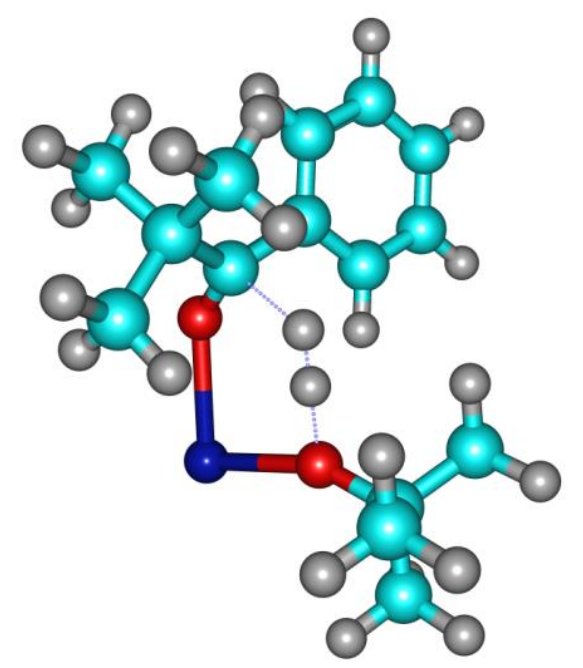

TS

$\begin{array}{lccc}\mathbf{G}=\mathbf{- 1 3 3 6 . 7 8 4 3 6 8} & \text { (Temperature }=\mathbf{4 8 3 . 1 5}, \mathbf{g a s}) \\ \mathbf{v}=\mathbf{9 6 6} \mathbf{~} \mathbf{m}^{-1} & & \\ \mathbf{E}=\mathbf{- 1 3 3 7 . 0 4 2 3 8 2 6 4} & \text { (gas) } & \\ 6 & 1.100299000 & -0.773379000 & 0.273441000 \\ 8 & 0.543242000 & -1.053014000 & 1.343858000 \\ 19 & -1.752113000 & -1.081521000 & 2.213719000 \\ 8 & -2.159600000 & 0.022962000 & 0.130036000 \\ 1 & -0.985078000 & -0.155994000 & -0.500456000 \\ 1 & -0.172138000 & -0.271885000 & -0.888239000 \\ 6 & 1.743829000 & 0.594175000 & 0.195133000 \\ 6 & 1.510466000 & -1.936606000 & -0.651164000 \\ 6 & -2.989654000 & 0.710942000 & -0.760076000 \\ 6 & 3.081650000 & 0.803132000 & -0.114018000 \\ 6 & 0.958363000 & 1.689283000 & 0.553246000 \\ 6 & 1.492735000 & 2.963379000 & 0.585462000 \\ 6 & 3.624201000 & 2.083314000 & -0.069265000 \\ 6 & 2.833637000 & 3.165219000 & 0.275473000 \\ 1 & 3.720806000 & -0.022716000 & -0.382983000 \\ 1 & 4.668258000 & 2.228102000 & -0.307935000 \\ 1 & 3.254304000 & 4.160302000 & 0.299283000 \\ 1 & 0.863539000 & 3.803766000 & 0.843228000 \\ 1 & -0.089338000 & 1.521529000 & 0.768369000 \\ 6 & 1.851763000 & -1.507798000 & -2.076108000 \\ 6 & 2.714158000 & -2.638408000 & 0.003710000 \\ 6 & 0.353664000 & -2.937273000 & -0.697677000 \\ 6 & -2.384646000 & 2.080919000 & -1.086439000 \\ 6 & -3.156904000 & -0.097760000 & -2.051432000 \\ 6 & -4.356725000 & 0.903407000 & -0.102130000 \\ 1 & 2.700998000 & -0.831456000 & -2.121307000 \\ 1 & -0.542290000 & -2.463676000 & -1.098959000 \\ 1 & 2.471332000 & -2.927337000 & 1.024858000 \\ 1 & -3.572409000 & -1.078422000 & -1.818171000\end{array}$
$-1.397109000$
$-4.245199000$
$-5.047793000$
$-4.793442000$
$-3.819089000$
$-2.187439000$
$-3.010204000$
$-2.272937000$
2.097302000
0.999870000
0.622745000
0.134259000
2.957498000
1.956094000
1.470895000
1.440667000
$-0.067946000$
0.402602000
$-0.243215000$
2.645085000
2.661376000
$-2.390659000$
$-1.012644000$
$-3.779031000$
$-3.320320000$
$-3.538005000$
$-1.531087000$
0.822919000
$-0.753153000$
0.134517000
$-2.759020000$
$-2.527541000$
$-1.779254000$
$-0.169442000$
$-2.666826000$
$-2.539488000$
$-1.335405000$
0.297200000
3.603564000
$-0.561876000$
0.030007000

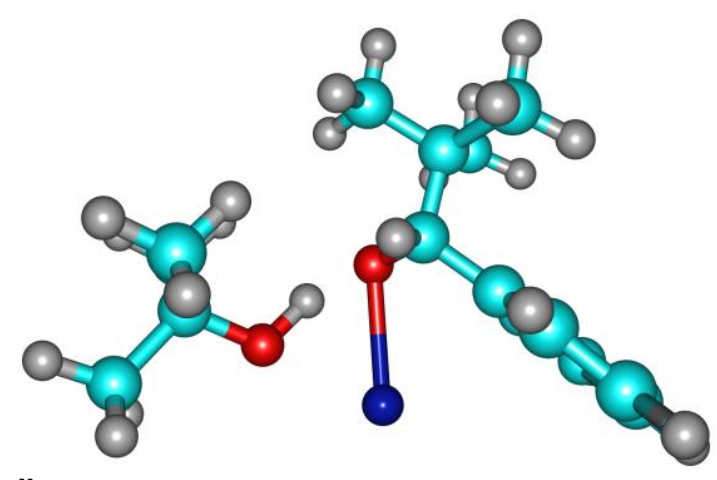

II

$\mathbf{G}=\mathbf{- 1 3 3 6 . 8 5 3 9 2 3}$ (Temperature=483.15, gas)

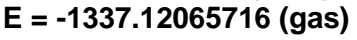

$\begin{array}{llll}6 & 0.493847000 & 0.807009000 & 0.104299000\end{array}$

$\begin{array}{llll}8 & -0.337312000 & 0.326453000 & -0.882055000\end{array}$

$\begin{array}{llll}19 & -0.185270000 & -2.221567000 & -0.799176000\end{array}$

$\begin{array}{llll}8 & -2.232615000 & -1.000588000 & -0.070412000\end{array}$

$\begin{array}{llll}8 & -1.511389000 & -0.285232000 & -0.385595000\end{array}$

$\begin{array}{llll}1 & 0.000083000 & 0.785219000 & 1.097297000\end{array}$

$\begin{array}{llll}6 & 1.724928000 & -0.091959000 & 0.243491000\end{array}$

$\begin{array}{llll}6 & 0.874614000 & 2.304943000 & -0.110733000\end{array}$

$\begin{array}{lll}-3.448253000 & -0.373226000 & 0.278370000\end{array}$

$\begin{array}{lll}2.375853000 & -0.569119000 & -0.897409000\end{array}$

$\begin{array}{lll}2.176498000 & -0.533073000 & 1.483888000\end{array}$

$\begin{array}{lll}3.243208000 & -1.419244000 & 1.590359000\end{array}$

$\begin{array}{lll}3.443150000 & -1.450677000 & -0.798657000\end{array}$

$\begin{array}{lll}3.880509000 & -1.882348000 & 0.449247000\end{array}$

$2.016500000 \quad-0.244091000-1.865507000$

$3.940621000-1.798193000-1.694045000$

$\begin{array}{lll}4.709627000 & -2.570758000 & 0.528274000\end{array}$

$\begin{array}{lll}3.572642000 & -1.750262000 & 2.565411000\end{array}$

$\begin{array}{lll}1.678439000 & -0.180569000 & 2.378498000\end{array}$

$\begin{array}{lll}1.803192000 & 2.789748000 & 1.000506000\end{array}$

$\begin{array}{lll}1.537844000 & 2.508228000 & -1.470642000\end{array}$

$\begin{array}{lll}-0.425505000 & 3.106202000 & -0.066001000\end{array}$

$\begin{array}{lll}-3.202615000 & 0.587538000 & 1.439890000\end{array}$

$\begin{array}{lll}-3.983649000 & 0.394246000 & -0.929267000\end{array}$

$\begin{array}{lll}-4.423328000 & -1.466746000 & 0.691652000\end{array}$

$\begin{array}{lll}2.770674000 & 2.288646000 & 0.964306000\end{array}$

$\begin{array}{lll}-0.891220000 & 3.031102000 & 0.918389000\end{array}$

$\begin{array}{lll}0.921453000 & 2.069153000 & -2.254233000\end{array}$

$-4.165553000 \quad-0.291923000 \quad-1.755939000$

$\begin{array}{lll}-2.485526000 & 1.351291000 & 1.138476000\end{array}$

$\begin{array}{lll}-4.025163000 & -2.020212000 & 1.541728000\end{array}$

$\begin{array}{lll}-5.388371000 & -1.046155000 & 0.972838000\end{array}$

$-4.576058000 \quad-2.162150000 \quad-0.133359000$

$\begin{array}{lll}-4.914709000 & 0.908821000 & -0.691630000\end{array}$

$\begin{array}{lll}-3.248023000 & 1.131293000 & -1.251670000\end{array}$

$\begin{array}{lll}-4.123375000 & 1.081103000 & 1.750988000\end{array}$

$\begin{array}{lll}-2.791399000 & 0.044369000 & 2.290521000\end{array}$

$\begin{array}{lll}1.978437000 & 3.862326000 & 0.907257000\end{array}$

$\begin{array}{lll}1.364310000 & 2.609136000 & 1.984236000\end{array}$

$\begin{array}{lll}-0.233573000 & 4.161292000 & -0.265182000\end{array}$

$\begin{array}{lll}-1.125507000 & 2.728855000 & -0.809429000\end{array}$

$\begin{array}{lll}1.655075000 & 3.573206000 & -1.675713000\end{array}$

$\begin{array}{lll}2.529792000 & 2.057442000 & -1.505776000\end{array}$ 


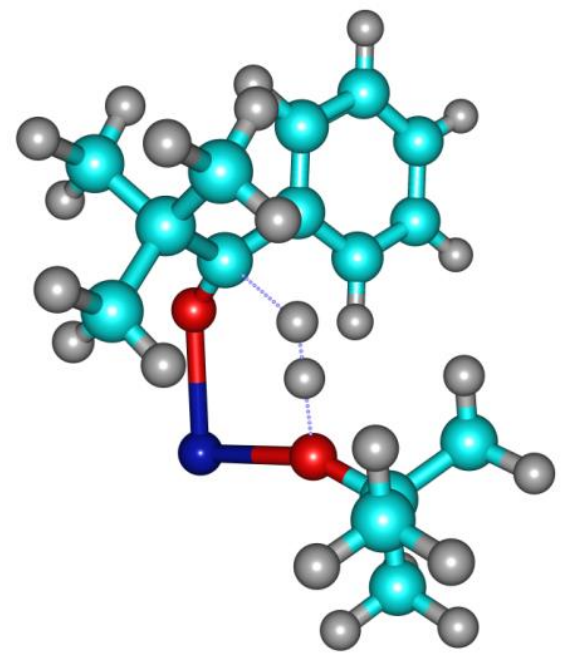

TS II

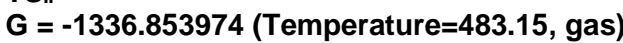

$\mathrm{v}=530 \mathrm{~cm}^{-1}$

$E=-1337.11953241$ (gas)

$\begin{array}{lrrr}6 & 0.476322000 & 0.837847000 & 0.096245000 \\ 8 & -0.346805000 & 0.397983000 & -0.932567000 \\ 19 & -0.187107000 & -2.261341000 & -0.685194000 \\ 8 & -2.125617000 & -0.951202000 & -0.072580000 \\ 1 & -1.301812000 & -0.144939000 & -0.496254000 \\ 1 & -0.051494000 & 0.777265000 & 1.064284000 \\ 6 & 1.686846000 & -0.083671000 & 0.215955000 \\ 6 & 0.864453000 & 2.334253000 & -0.072436000 \\ 6 & -3.364395000 & -0.384568000 & 0.241370000 \\ 6 & 2.333214000 & -0.542631000 & -0.934062000 \\ 6 & 2.116180000 & -0.567673000 & 1.447903000 \\ 6 & 3.159817000 & -1.482620000 & 1.536900000 \\ 6 & 3.377331000 & -1.452987000 & -0.851651000 \\ 6 & 3.793253000 & -1.929705000 & 0.387126000 \\ 1 & 1.984494000 & -0.187346000 & -1.895052000 \\ 1 & 3.871309000 & -1.789977000 & -1.752906000 \\ 1 & 4.603783000 & -2.641285000 & 0.453142000 \\ 1 & 3.474093000 & -1.848926000 & 2.504251000 \\ 1 & 1.617613000 & -0.229929000 & 2.347921000 \\ 6 & 1.794328000 & 2.773238000 & 1.056956000 \\ 6 & 1.536013000 & 2.574069000 & -1.422126000 \\ 6 & -0.428900000 & 3.145174000 & -0.004187000 \\ 6 & -3.203948000 & 0.585758000 & 1.413989000 \\ 6 & -3.907375000 & 0.369293000 & -0.975467000 \\ 6 & -4.326845000 & -1.504275000 & 0.629363000 \\ 1 & 2.758188000 & 2.266246000 & 1.006570000 \\ 1 & -0.893275000 & 3.049775000 & 0.978715000 \\ 1 & 0.914226000 & 2.183075000 & -2.226451000 \\ 1 & -4.033993000 & -0.320766000 & -1.809857000 \\ 1 & -2.517770000 & 1.386242000 & 1.136004000 \\ 1 & -3.936928000 & -2.043584000 & 1.493038000 \\ 1 & -5.314823000 & -1.116375000 & 0.879800000 \\ 1 & -4.430097000 & -2.206324000 & -0.198271000 \\ 1 & -4.868086000 & 0.840535000 & -0.764629000 \\ 1 & -3.197818000 & 1.140447000 & -1.277351000 \\ 1 & -4.154949000 & 1.033404000 & 1.705548000 \\ 1 & -2.787702000 & 0.058602000 & 2.273017000 \\ 1 & 1.977786000 & 3.846573000 & 0.996941000 \\ 1 & 1.351206000 & 2.566489000 & 2.033545000 \\ 1 & -0.227085000 & 4.202633000 & -0.178643000 \\ 1 & -1.136182000 & 2.795205000 & -0.754228000 \\ 1 & 1.678720000 & 3.643344000 & -1.582659000 \\ 1 & 2.517248000 & 2.101844000 & -1.471876000\end{array}$

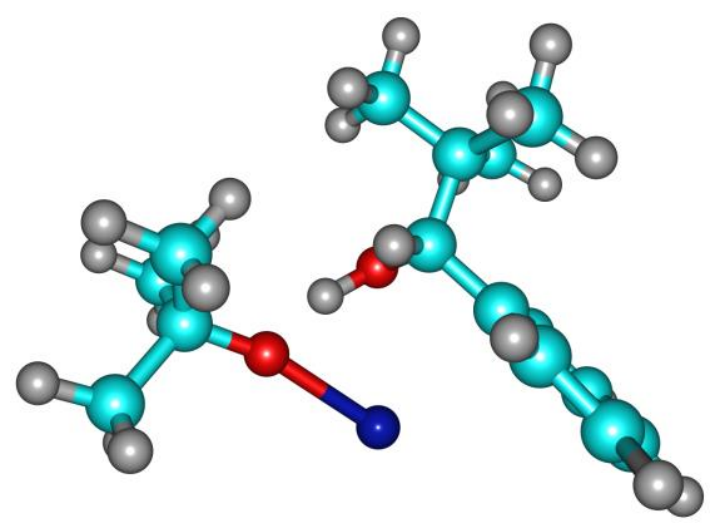

III

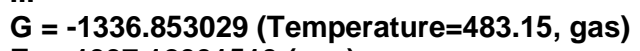

$E=\mathbf{- 1 3 3 7 . 1 2 0 0 1 5 1 0 ~ ( g a s ) ~}$

$\begin{array}{llll}6 & 0.513429000 & 0.890457000 & 0.068743000\end{array}$

$\begin{array}{llll}8 & -0.293390000 & 0.542600000 & -1.020175000\end{array}$

$19-0.305357000 \quad-2.204275000-0.869682000$

$\begin{array}{llll}8 & -2.112175000 & -0.839813000 & -0.156570000\end{array}$

$\begin{array}{llll}8 & -1.182149000 & 0.063435000 & -0.643280000\end{array}$

$\begin{array}{llll}1 & -0.080371000 & 0.857851000 & 0.993809000\end{array}$

$\begin{array}{llll}6 & 1.622567000 & -0.140098000 & 0.230806000\end{array}$

$\begin{array}{llll}6 & 1.030796000 & 2.346763000 & -0.056794000\end{array}$

$\begin{array}{llll}6 & -3.324242000 & -0.305983000 & 0.263382000\end{array}$

$\begin{array}{llll}6 & 2.315892000 & -0.613254000 & -0.885822000\end{array}$

$\begin{array}{llll}6 & 1.907464000 & -0.704980000 & 1.469934000\end{array}$

$\begin{array}{llll}6 & 2.857230000 & -1.712671000 & 1.598920000\end{array}$

$\begin{array}{llll}6 & 3.266799000 & -1.616533000 & -0.762745000\end{array}$

$\begin{array}{llll}6 & 3.539519000 & -2.172789000 & 0.482667000\end{array}$

$\begin{array}{llll}1 & 2.080373000 & -0.197737000 & -1.856620000\end{array}$

$\begin{array}{llll}1 & 3.799747000 & -1.964080000 & -1.637145000\end{array}$

$\begin{array}{llll}1 & 4.277835000 & -2.955700000 & 0.579489000\end{array}$

$\begin{array}{llll}1 & 3.058580000 & -2.140667000 & 2.570820000\end{array}$

$\begin{array}{llll}1 & 1.366956000 & -0.358996000 & 2.341757000\end{array}$

$\begin{array}{llll}6 & 1.923841000 & 2.687463000 & 1.134962000\end{array}$

$\begin{array}{llll}6 & 1.803213000 & 2.549987000 & -1.358807000\end{array}$

$\begin{array}{lrrr}6 & 1.803213000 & 2.549987000 & -1.358807000 \\ 6 & -0.191803000 & 3.264541000 & -0.050108000\end{array}$

$\begin{array}{llll}6 & -3.082055000 & 0.749673000 & 1.348214000\end{array}$

$\begin{array}{llll}6 & -4.043062000 & 0.348587000 & -0.921934000\end{array}$

$\begin{array}{llll}6 & -4.196407000 & -1.426565000 & 0.833553000\end{array}$

$\begin{array}{llll}1 & 2.836343000 & 2.091225000 & 1.138089000\end{array}$

$\begin{array}{llll}1 & -0.731778000 & 3.182075000 & 0.894271000\end{array}$

$\begin{array}{llll}1 & 1.207540000 & 2.220034000 & -2.208968000\end{array}$

$\begin{array}{llll}1 & -4.226802000 & -0.396650000 & -1.696147000\end{array}$

$\begin{array}{llll}1 & -2.473865000 & 1.558790000 & 0.941351000\end{array}$

$\begin{array}{llll}1 & -3.687319000 & -1.894678000 & 1.676666000\end{array}$

$1-5.165451000 \quad-1.058844000 \quad 1.173798000$

$\begin{array}{llll}1 & -4.363863000 & -2.185486000 & 0.068497000\end{array}$

$\begin{array}{llll}1 & -4.996887000 & 0.790716000 & -0.630282000\end{array}$

$\begin{array}{llll}1 & -3.411824000 & 1.130688000 & -1.345513000\end{array}$

$1 \quad-4.013575000 \quad 1.175861000 \quad 1.723346000$

$\begin{array}{llll}1 & -2.542851000 & 0.299321000 & 2.182698000\end{array}$

$\begin{array}{llll}1 & 2.210509000 & 3.738778000 & 1.100243000\end{array}$

$\begin{array}{llll}1 & 1.401878000 & 2.515731000 & 2.078414000\end{array}$

$1 \quad 0.113599000 \quad 4.303971000 \quad-0.172993000$

$\begin{array}{llll}1 & -0.871957000 & 3.001327000 & -0.858006000\end{array}$

$1 \quad 2.034437000 \quad 3.607413000 \quad-1.490497000$

$\begin{array}{llll}1 & 2.748110000 & 2.006951000 & -1.355703000\end{array}$ 


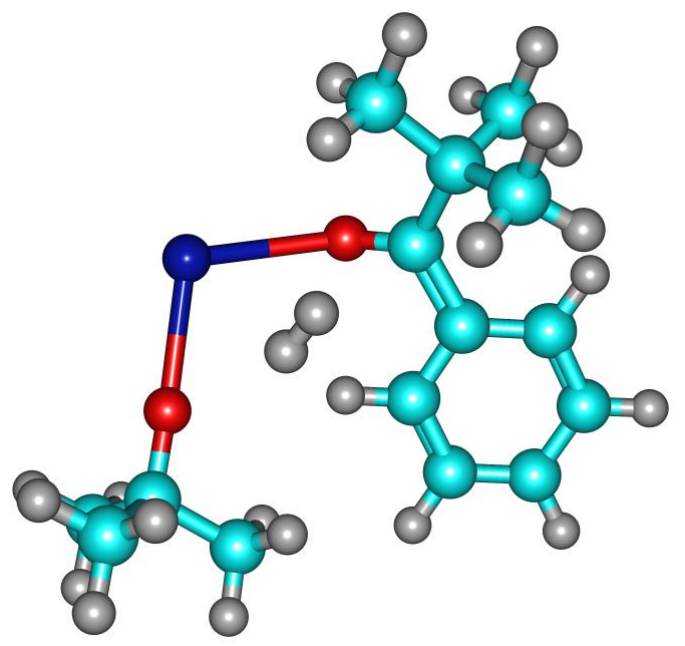

12

$\begin{array}{lrrr}\mathbf{G}=\mathbf{- 1 3 3 6 . 8 2 9 9 5 7} \text { (Temperature=483.15, pressure }=\mathbf{3 9 . 6 4 )} \\ \mathbf{E}=\mathbf{- 1 3 3 7 . 0 9 0 1 4 8 1 8} & & \\ 6 & -1.793856000 & -0.492118000 & -0.395420000 \\ 8 & -0.996687000 & -1.086716000 & -1.098533000 \\ 19 & 1.204698000 & -2.380198000 & -0.685659000 \\ 8 & 2.825976000 & -0.739168000 & -0.142772000 \\ 1 & 0.737588000 & -0.782518000 & 1.512286000 \\ 1 & 0.076472000 & -1.023420000 & 1.754229000 \\ 6 & -1.656334000 & 0.994226000 & -0.286086000 \\ 6 & -2.867665000 & -1.279011000 & 0.358950000 \\ 6 & 3.797208000 & 0.161273000 & 0.239891000 \\ 6 & -2.735197000 & 1.866769000 & -0.152370000 \\ 6 & -0.362068000 & 1.513986000 & -0.355479000 \\ 6 & -0.150938000 & 2.880264000 & -0.274843000 \\ 6 & -2.521828000 & 3.235958000 & -0.100624000 \\ 6 & -1.231504000 & 3.744046000 & -0.153033000 \\ 1 & -3.746462000 & 1.494818000 & -0.113353000 \\ 1 & -3.366016000 & 3.905513000 & -0.016314000 \\ 1 & -1.067995000 & 4.811382000 & -0.099138000 \\ 1 & 0.856592000 & 3.270561000 & -0.309504000 \\ 1 & 0.480051000 & 0.839728000 & -0.456062000 \\ 6 & -3.148361000 & -0.692225000 & 1.744852000 \\ 6 & -4.143643000 & -1.292222000 & -0.499558000 \\ 6 & -2.385911000 & -2.722052000 & 0.523538000 \\ 6 & 3.228102000 & 1.589363000 & 0.268335000 \\ 6 & 4.325621000 & -0.173720000 & 1.645274000 \\ 6 & 4.982789000 & 0.134113000 & -0.740108000 \\ 1 & -3.810448000 & -1.369212000 & 2.284620000 \\ 1 & -1.477742000 & -2.767696000 & 1.127104000 \\ 1 & -3.941870000 & -1.716033000 & -1.483168000 \\ 1 & 4.749197000 & -1.180144000 & 1.650777000 \\ 1 & 2.397236000 & 1.642282000 & 0.975595000 \\ 1 & 4.635554000 & 0.381791000 & -1.745290000 \\ 1 & 5.771631000 & 0.838768000 & -0.466724000 \\ 1 & 5.414849000 & -0.868240000 & -0.770011000 \\ 1 & 5.096286000 & 0.523289000 & 1.982771000 \\ 1 & 3.504437000 & -0.149186000 & 2.364879000 \\ 1 & 3.972688000 & 2.334207000 & 0.559577000 \\ 1 & 2.848746000 & 1.853110000 & -0.721339000 \\ 1 & -2.228888000 & -0.591043000 & 2.322999000 \\ 1 & -3.631333000 & 0.280096000 & 1.702741000 \\ 1 & -3.154395000 & -3.296581000 & 1.039209000 \\ 1 & -2.195409000 & -3.194349000 & -0.437712000 \\ 1 & -4.889496000 & -1.915612000 & -0.005727000 \\ 1 & -4.573323000 & -0.302590000 & -0.634399000 \\ & & & \\ & & & \\ 1 & & \end{array}$

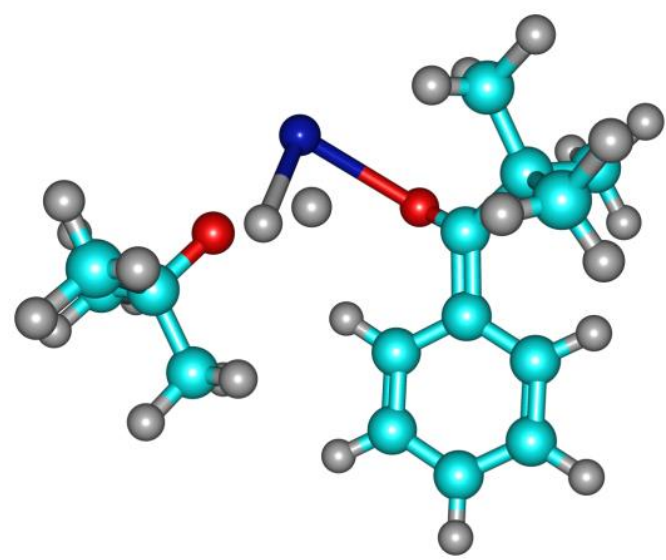

$\mathrm{TS}_{7}$

$\mathrm{G}=\mathbf{- 1 3 3 6 . 8 1 2 8 1 9}($ Temperature $=483.15$, pressure $=39.64$ )

$\mathrm{v}=1553 \mathrm{~cm}^{-1}$

\section{$E=\mathbf{- 1 3 3 7 . 0 7 3 3 7 9 5 3}$}

$\begin{array}{llll}6 & -1.725214000 & -0.511409000 & -0.432869000\end{array}$

$8 \quad-1.024812000 \quad-1.054079000 \quad-1.269491000$

$\begin{array}{lllll}19 & 1.131723000 & -2.422013000 & -0.874482000\end{array}$

$\begin{array}{lll}2.662090000 & -0.654631000 & -0.065665000\end{array}$

$\begin{array}{lll}1.716757000 & -0.706417000 & 0.762064000\end{array}$

$\begin{array}{lll}0.909728000 & -0.870290000 & 1.327876000\end{array}$

$\begin{array}{lll}-1.663647000 & 0.979683000 & -0.327950000\end{array}$

$\begin{array}{lll}-2.619771000 & -1.356347000 & 0.474760000\end{array}$

$\begin{array}{lll}3.641556000 & 0.269889000 & 0.333534000\end{array}$

$\begin{array}{lll}-2.770702000 & 1.785814000 & -0.068755000\end{array}$

$\begin{array}{lll}-0.418143000 & 1.573272000 & -0.535068000\end{array}$

$\begin{array}{lll}-0.278673000 & 2.949645000 & -0.470223000\end{array}$

$\begin{array}{lll}-2.633124000 & 3.165412000 & -0.033828000\end{array}$

$\begin{array}{lll}-1.388247000 & 3.748445000 & -0.226291000\end{array}$

$\begin{array}{lll}-3.746551000 & 1.351772000 & 0.084191000\end{array}$

$\begin{array}{lll}-3.500017000 & 3.784408000 & 0.149186000\end{array}$

$\begin{array}{lll}-1.282525000 & 4.823570000 & -0.183975000\end{array}$

$\begin{array}{lll}0.694881000 & 3.398829000 & -0.609471000\end{array}$

$\begin{array}{lll}0.442590000 & 0.944237000 & -0.721192000\end{array}$

$\begin{array}{lll}-2.763294000 & -0.750307000 & 1.872765000\end{array}$

$\begin{array}{lll}-3.989234000 & -1.490769000 & -0.212552000\end{array}$

$\begin{array}{lll}-2.003842000 & -2.750802000 & 0.607765000\end{array}$

$\begin{array}{lll}3.057947000 & 1.685304000 & 0.321215000\end{array}$

$\begin{array}{lll}4.136139000 & -0.065528000 & 1.743743000\end{array}$

$\begin{array}{lll}4.810701000 & 0.194450000 & -0.646093000\end{array}$

$\begin{array}{lll}-3.277339000 & -1.465467000 & 2.514926000\end{array}$

$\begin{array}{lll}-1.037606000 & -2.696319000 & 1.113199000\end{array}$

$\begin{array}{lll}-3.884214000 & -1.959091000 & -1.191058000\end{array}$

$\begin{array}{lll}4.536738000 & -1.080104000 & 1.767377000\end{array}$

$\begin{array}{lll}2.208347000 & 1.748977000 & 1.002859000\end{array}$

$\begin{array}{lll}4.468738000 & 0.420617000 & -1.657161000\end{array}$

$\begin{array}{lll}5.598279000 & 0.902850000 & -0.383356000\end{array}$

$\begin{array}{lll}5.238088000 & -0.809354000 & -0.645708000\end{array}$

$\begin{array}{lll}4.920431000 & 0.621318000 & 2.065749000\end{array}$

$\begin{array}{lll}3.315526000 & -0.006964000 & 2.459272000\end{array}$

$\begin{array}{lll}3.798253000 & 2.427957000 & 0.623756000\end{array}$

$\begin{array}{lll}2.711347000 & 1.935410000 & -0.683056000\end{array}$

$\begin{array}{lll}-1.784920000 & -0.546314000 & 2.309513000\end{array}$

$\begin{array}{lll}-3.338441000 & 0.171441000 & 1.875660000\end{array}$

$\begin{array}{lll}-2.658263000 & -3.373754000 & 1.216131000\end{array}$

$\begin{array}{lll}-1.886523000 & -3.233370000 & -0.360935000\end{array}$

$\begin{array}{lll}-4.629485000 & -2.122887000 & 0.403307000\end{array}$

$\begin{array}{lll}-4.489488000 & -0.533650000 & -0.343649000\end{array}$ 


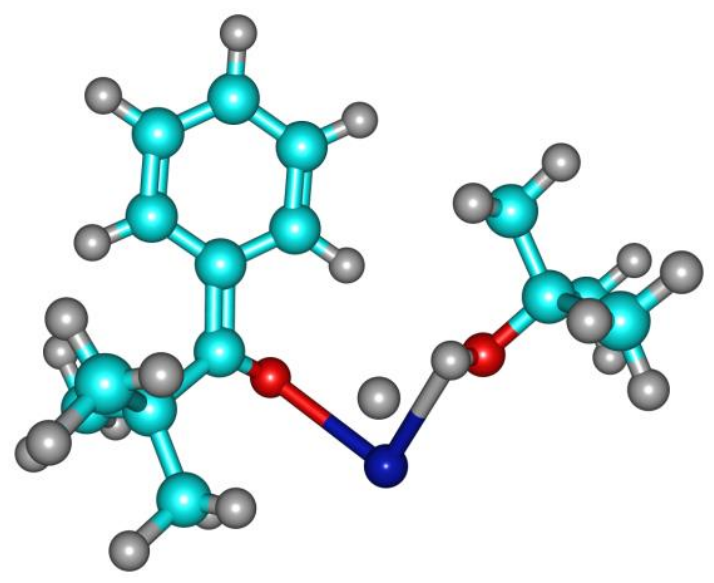

13

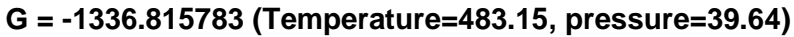

\section{$E=-1337.07990519$}

$6-1.734902000$

$8-0.956893000$

$19 \quad 1.031730000$

$-0.466305000$

$-1.026802000$

$-0.423665000$

2.695587000

$-2.562584000$

$-0.670822000$

2.053027000

0.873108000

$-1.634960000$

$-2.762566000$

3.759717000

$-2.736137000$

$-0.354612000$

$-0.177120000$

$-2.557346000$

$-1.279689000$

$-3.737608000$

$-3.418577000$

$-1.143103000$

0.820426000

0.498955000

$-3.026880000$

$-4.055085000$

$-2.229739000$

3.193348000

4.437378000

4.729770000

$-3.639415000$

$-1.324968000$

$-3.863640000$

4.817329000

2.449132000

4.228795000

5.567080000

5.122581000

5.270966000

3.726702000

3.981979000

2.717148000

$-2.091365000$

$-3.555666000$

$-2.976138000$

$-2.029708000$

$-4.775932000$

$-4.510571000$
$-0.667077000$

$-1.075967000$

1.021188000

$-1.284652000$

0.248278000

1.863375000

1.573484000

2.942871000

3.236670000

3.777495000

1.464173000

3.883395000

4.847312000

3.359358000

0.916080000

$-0.696571000$

$-1.344573000$

$-2.709132000$

1.661700000

$-0.120193000$

0.115515000

$-1.396973000$

$-2.708356000$

$-1.781947000$

$-1.141090000$

1.732194000

0.344841000

0.802450000

$-0.899998000$

0.550862000

$-0.052593000$

2.387423000

1.921152000

$-0.549126000$

0.252116000

$-3.310164000$

$-3.179827000$

$-1.975407000$

$-0.367651000$
$-1.175524000$

$-0.555730000$

$-0.033883000$

0.735658000

1.644280000

$-0.292496000$

0.360127000

0.221237000

$-0.342923000$

$-0.236949000$

$-0.076100000$

$-0.108759000$

$-0.124786000$

0.014235000

$-0.033035000$

$-0.253463000$

$-0.441340000$

1.748415000

$-0.471077000$

0.527811000

0.289204000

1.535766000

$-0.940470000$

2.316204000

1.138648000

$-1.450962000$

1.491994000

1.084195000

$-1.881223000$

$-0.820149000$

$-0.994941000$

1.743504000

2.360141000

0.489482000

$-0.656936000$

2.289536000

1.711353000

1.045563000

$-0.433507000$

0.049756000

$-0.615389000$
$-0.152568000$

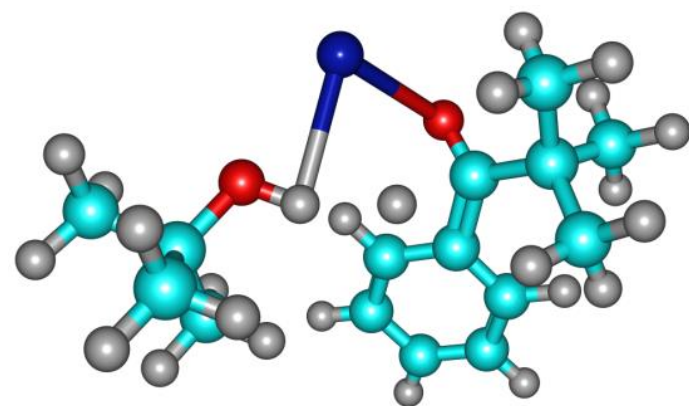

$\mathrm{TS}_{8}$

$\begin{array}{lrrr}\mathbf{G}=\mathbf{- 1 3 3 6 . 8 0 9 3 6 5} \text { (Temperature=483.15, pressure=39.64) } \\ \mathbf{v}=\mathbf{6 3 2} \mathbf{~ c m}^{-1} & & \\ \mathbf{E}=\mathbf{- 1 3 3 7 . 0 7 2 0 0 5 0 2} & & \\ 6 & -1.558980000 & -0.480006000 & -0.373412000 \\ 8 & -1.226623000 & -0.986303000 & -1.451002000 \\ 19 & 0.859932000 & -2.398302000 & -1.114817000 \\ 8 & 2.478197000 & -0.632256000 & -0.143248000 \\ 1 & 1.625206000 & -0.533968000 & 0.374552000 \\ 1 & 0.183586000 & -0.763733000 & 0.805636000 \\ 6 & -1.446310000 & 1.024121000 & -0.276290000 \\ 6 & -2.448982000 & -1.307452000 & 0.573041000 \\ 6 & 3.494034000 & 0.170348000 & 0.464205000 \\ 6 & -2.437028000 & 1.842816000 & 0.255563000 \\ 6 & -0.329820000 & 1.624506000 & -0.861335000 \\ 6 & -0.192310000 & 2.999347000 & -0.890360000 \\ 6 & -2.305619000 & 3.227980000 & 0.218248000 \\ 6 & -1.184319000 & 3.810296000 & -0.346599000 \\ 1 & -3.331423000 & 1.425006000 & 0.686294000 \\ 1 & -3.089422000 & 3.846730000 & 0.632683000 \\ 1 & -1.079965000 & 4.886186000 & -0.366976000 \\ 1 & 0.689503000 & 3.443156000 & -1.331807000 \\ 1 & 0.446207000 & 0.993256000 & -1.272954000 \\ 6 & -2.493249000 & -0.825358000 & 2.022581000 \\ 6 & -3.863462000 & -1.275033000 & -0.040401000 \\ 6 & -1.975755000 & -2.762015000 & 0.562513000 \\ 6 & 3.066349000 & 1.632454000 & 0.423697000 \\ 6 & 3.697486000 & -0.285662000 & 1.904392000 \\ 6 & 4.756784000 & -0.044036000 & -0.352508000 \\ 1 & -2.999333000 & -1.579936000 & 2.626036000 \\ 1 & -1.004148000 & -2.848652000 & 1.051629000 \\ 1 & -3.855345000 & -1.697819000 & -1.044563000 \\ 1 & 3.985589000 & -1.336703000 & 1.931762000 \\ 1 & 2.132683000 & 1.767946000 & 0.971847000 \\ 1 & 4.594784000 & 0.255357000 & -1.388188000 \\ 1 & 5.578522000 & 0.546242000 & 0.052034000 \\ 1 & 5.047124000 & -1.094704000 & -0.336342000 \\ 1 & 4.478019000 & 0.299965000 & 2.390255000 \\ 1 & 2.773847000 & -0.166424000 & 2.472092000 \\ 1 & 3.826028000 & 2.271662000 & 0.874056000 \\ 1 & 2.912004000 & 1.952615000 & -0.607200000 \\ 1 & -1.487641000 & -0.693066000 & 2.418505000 \\ 1 & -3.038916000 & 0.104543000 & 2.151479000 \\ 1 & -2.683400000 & -3.377338000 & 1.117537000 \\ 1 & -1.921919000 & -3.157164000 & -0.451340000 \\ 1 & -4.533704000 & -1.872757000 & 0.577939000 \\ 1 & -4.272092000 & -0.268029000 & -0.099453000 \\ & & & \end{array}$

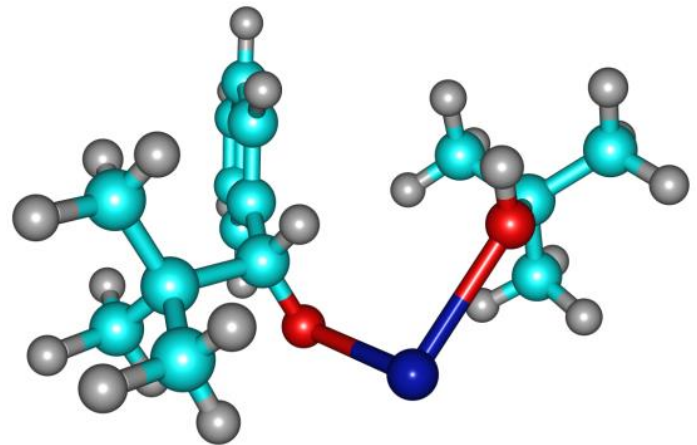




$\begin{array}{lrrr}\mathbf{G}=\mathbf{- 1 3 3 6 . 8 6 0 6 3 3} \text { (Temperature=483.15, pressure }=\mathbf{3 9 . 6} \text { ) } \\ \mathbf{E}=\mathbf{- 1 3 3 7 . 1 3 1 6 1 4 2 8} & & \\ 6 & -0.918682000 & -0.664539000 & -0.062258000 \\ 8 & -0.325962000 & -1.009686000 & -1.241389000 \\ 19 & 1.713776000 & -2.215723000 & -0.548499000 \\ 8 & 2.977082000 & -0.373010000 & 0.826717000 \\ 1 & 2.805766000 & -0.198159000 & 1.758454000 \\ 1 & -0.184091000 & -0.617650000 & 0.783540000 \\ 6 & -1.537530000 & 0.735020000 & -0.101257000 \\ 6 & -1.962949000 & -1.734429000 & 0.433171000 \\ 6 & 3.258090000 & 0.883116000 & 0.174296000 \\ 6 & -2.102918000 & 1.219379000 & -1.278075000 \\ 6 & -1.527671000 & 1.573020000 & 1.012247000 \\ 6 & -2.075796000 & 2.848262000 & 0.961361000 \\ 6 & -2.658838000 & 2.491034000 & -1.336604000 \\ 6 & -2.648573000 & 3.312081000 & -0.215876000 \\ 1 & -2.090366000 & 0.590231000 & -2.157554000 \\ 1 & -3.094491000 & 2.845885000 & -2.261298000 \\ 1 & -3.072582000 & 4.305827000 & -0.262166000 \\ 1 & -2.048454000 & 3.482741000 & 1.837361000 \\ 1 & -1.071006000 & 1.225777000 & 1.931734000 \\ 6 & -2.533937000 & -1.374526000 & 1.803526000 \\ 6 & -3.105167000 & -1.886273000 & -0.567152000 \\ 6 & -1.228705000 & -3.069697000 & 0.557772000 \\ 6 & 2.048994000 & 1.793654000 & 0.314888000 \\ 6 & 4.495739000 & 1.498030000 & 0.808282000 \\ 6 & 3.503163000 & 0.541737000 & -1.285334000 \\ 1 & -3.163162000 & -2.185545000 & 2.175805000 \\ 1 & -0.402097000 & -2.993069000 & 1.272221000 \\ 1 & -2.725153000 & -2.113848000 & -1.563051000 \\ 1 & 5.348575000 & 0.826806000 & 0.708373000 \\ 1 & 1.833746000 & 1.985958000 & 1.367523000 \\ 1 & 2.587706000 & 0.181614000 & -1.759090000 \\ 1 & 3.816340000 & 1.430777000 & -1.830192000 \\ 1 & 4.288783000 & -0.208791000 & -1.379790000 \\ 1 & 4.741584000 & 2.445656000 & 0.330571000 \\ 1 & 4.324774000 & 1.686455000 & 1.869262000 \\ 1 & 2.227833000 & 2.750343000 & -0.175476000 \\ 1 & 1.175683000 & 1.329241000 & -0.144203000 \\ 1 & -1.738487000 & -1.211284000 & 2.534566000 \\ 1 & -3.145637000 & -0.473722000 & 1.764531000 \\ 1 & -1.897049000 & -3.848374000 & 0.928715000 \\ 1 & -0.847046000 & -3.397109000 & -0.409544000 \\ 1 & -3.770134000 & -2.698421000 & -0.265536000 \\ 1 & -3.703065000 & -0.976257000 & -0.629817000 \\ & & & \end{array}$

$\begin{array}{rrrr}6 & -3.026733000 & 0.098715000 & 0.047266000 \\ 6 & 0.735451000 & 1.740334000 & 1.063441000 \\ 6 & 1.066258000 & 1.794800000 & -1.303783000 \\ 6 & 0.905731000 & 3.175025000 & -1.300552000 \\ 6 & 0.579512000 & 3.119563000 & 1.075435000 \\ 6 & 0.660785000 & 3.843187000 & -0.108607000 \\ 1 & 0.642587000 & 1.176048000 & 1.981452000 \\ 1 & 0.383622000 & 3.631126000 & 2.008419000 \\ 1 & 0.527281000 & 4.916250000 & -0.102365000 \\ 1 & 0.960639000 & 3.726506000 & -2.229560000 \\ 1 & 1.237983000 & 1.278606000 & -2.240813000 \\ 6 & 3.505551000 & -0.389836000 & -0.943385000 \\ 6 & 3.051750000 & -0.576950000 & 1.501486000 \\ 6 & 2.552017000 & -2.485019000 & -0.014596000 \\ 6 & -2.659624000 & 1.380749000 & -0.696541000 \\ 6 & -4.389481000 & -0.400550000 & -0.410553000 \\ 6 & -3.030244000 & 0.351473000 & 1.551924000 \\ 1 & 4.503465000 & -0.815573000 & -0.824275000 \\ 1 & 2.182349000 & -2.794764000 & -0.997657000 \\ 1 & 2.367690000 & -0.930970000 & 2.272673000 \\ 1 & -4.654453000 & -1.318143000 & 0.115739000 \\ 1 & -2.598912000 & 1.189046000 & -1.768617000 \\ 1 & -2.044901000 & 0.684237000 & 1.882024000 \\ 1 & -3.756847000 & 1.120199000 & 1.816380000 \\ 1 & -3.283081000 & -0.562623000 & 2.090349000 \\ 1 & -5.162491000 & 0.342889000 & -0.216545000 \\ 1 & -4.374610000 & -0.608913000 & -1.481048000 \\ 1 & -3.405704000 & 2.157968000 & -0.526816000 \\ 1 & -1.692521000 & 1.755229000 & -0.357772000 \\ 1 & 3.162932000 & -0.623447000 & -1.953948000 \\ 1 & 3.595167000 & 0.693044000 & -0.859568000 \\ 1 & 3.561224000 & -2.886402000 & 0.086979000 \\ 1 & 1.939090000 & -2.936726000 & 0.765791000 \\ 1 & 4.031177000 & -1.021316000 & 1.689176000 \\ 1 & 3.154795000 & 0.503566000 & 1.604189000\end{array}$

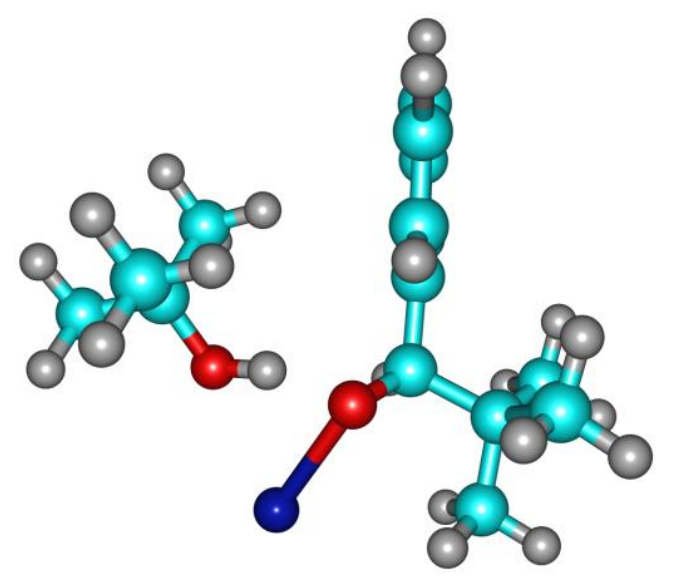

15

$\mathrm{G}=\mathbf{- 1 3 3 6 . 8 7 0 6 3 3}($ Temperature $=483.15$, pressure $=39.64$ )

$E=-1337.14102117$

$\begin{array}{llll}6 & 1.095765000 & -0.463160000 & -0.130865000\end{array}$

$\begin{array}{llll}8 & 0.198591000 & -1.037572000 & 0.745264000\end{array}$

$19 \quad-0.704715000 \quad-3.113779000 \quad-0.396916000$

$8 \quad-2.088661000 \quad-0.922765000-0.262077000$

$\begin{array}{llll}1 & -1.180810000 & -0.784476000 & 0.219665000\end{array}$

$\begin{array}{llll}1 & 0.882169000 & -0.759938000 & -1.182993000\end{array}$

$\begin{array}{llll}6 & 0.984441000 & 1.057968000 & -0.125728000\end{array}$

$\begin{array}{llll}6 & 2.557542000 & -0.960786000 & 0.109232000\end{array}$

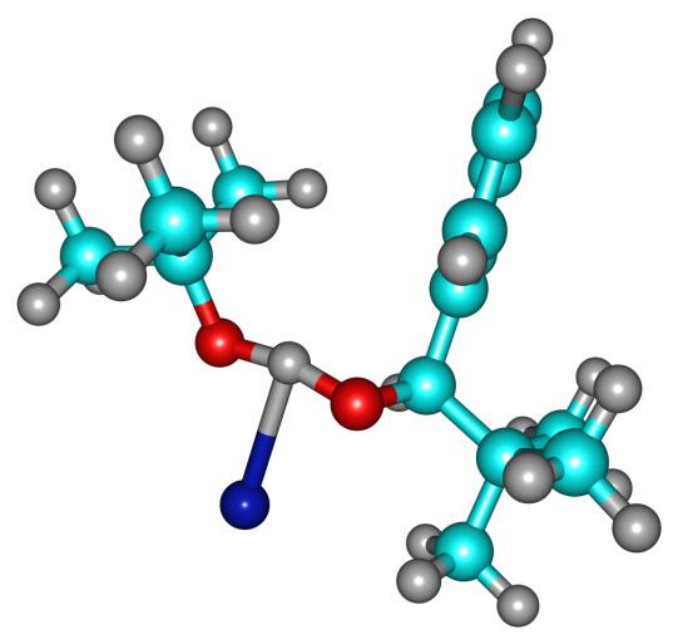

TS9

$\mathrm{G}=-\mathbf{1 3 3 6 . 8 6 8 4 6 8}($ Temperature $=483.15$, pressure $=39.64)$

$\mathrm{v}=878 \mathrm{~cm}^{-1}$

$E=-1337.13931555$

$\begin{array}{lccc}6 & 1.000309000 & -0.573844000 & -0.141495000 \\ 8 & 0.010136000 & -0.995910000 & 0.745483000 \\ 19 & -1.251778000 & -2.980978000 & -0.428247000 \\ 8 & -2.153575000 & -0.653101000 & -0.218144000 \\ 1 & -1.052093000 & -0.682525000 & 0.318953000 \\ 1 & 0.726703000 & -0.838514000 & -1.181132000 \\ 6 & 1.144025000 & 0.940008000 & -0.132672000 \\ 6 & 2.336512000 & -1.322591000 & 0.126086000 \\ 6 & -2.933372000 & 0.482624000 & 0.053045000 \\ 6 & 1.010137000 & 1.659666000 & 1.052275000 \\ 6 & 1.363933000 & 1.642624000 & -1.313771000 \\ 6 & 1.450974000 & 3.028695000 & -1.316847000 \\ 6 & 1.096983000 & 3.045137000 & 1.056106000 \\ 6 & 1.316278000 & 3.735612000 & -0.129542000 \\ 1 & 0.817530000 & 1.127727000 & 1.973882000 \\ 1 & 0.985613000 & 3.588135000 & 1.985024000\end{array}$




$\begin{array}{rrrr}1 & 1.374346000 & 4.815332000 & -0.128576000 \\ 1 & 1.611362000 & 3.556626000 & -2.247129000 \\ 1 & 1.448821000 & 1.098401000 & -2.246588000 \\ 6 & 3.384327000 & -0.928569000 & -0.913310000 \\ 6 & 2.869878000 & -1.024558000 & 1.525427000 \\ 6 & 2.067942000 & -2.823629000 & 0.001345000 \\ 6 & -2.445498000 & 1.671703000 & -0.778793000 \\ 6 & -4.382959000 & 0.169669000 & -0.312432000 \\ 6 & -2.843713000 & 0.836469000 & 1.539189000 \\ 1 & 4.291267000 & -1.518474000 & -0.772748000 \\ 1 & 1.658316000 & -3.064776000 & -0.984662000 \\ 1 & 2.123951000 & -1.247247000 & 2.288191000 \\ 1 & -4.745416000 & -0.675074000 & 0.275434000 \\ 1 & -2.457922000 & 1.415356000 & -1.839551000 \\ 1 & -1.810484000 & 1.058738000 & 1.811076000 \\ 1 & -3.454468000 & 1.707673000 & 1.780825000 \\ 1 & -3.184851000 & -0.002373000 & 2.148054000 \\ 1 & -5.037777000 & 1.022272000 & -0.126659000 \\ 1 & -4.454383000 & -0.092997000 & -1.369159000 \\ 1 & -3.075109000 & 2.550598000 & -0.630008000 \\ 1 & -1.422666000 & 1.934217000 & -0.502646000 \\ 1 & 3.023381000 & -1.108015000 & -1.928279000 \\ 1 & 3.656473000 & 0.123388000 & -0.832224000 \\ 1 & 2.994715000 & -3.388569000 & 0.107212000 \\ 1 & 1.385633000 & -3.168628000 & 0.778452000 \\ 1 & 3.751633000 & -1.635420000 & 1.726608000 \\ 1 & 3.162183000 & 0.020715000 & 1.625967000\end{array}$

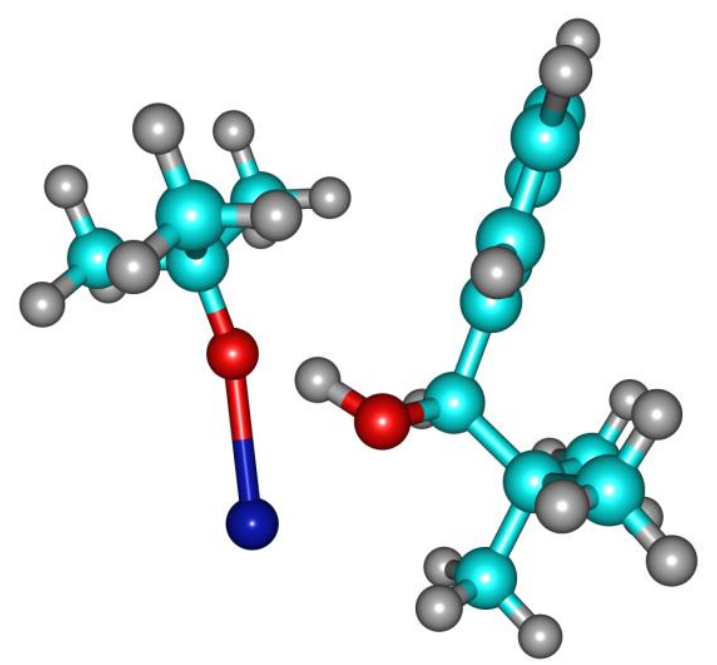

16

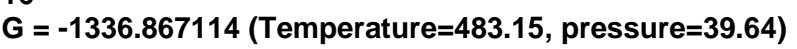
$E=\mathbf{- 1 3 3 7 . 1 3 9 7 8 4 7 0}$

$6 \quad 0.997773000-0.601987000$

$\begin{array}{lllll}8 & 0.014835000 & -0.993881000 & 0.779322000\end{array}$

$19 \quad-1.444473000 \quad-2.919104000 \quad-0.359388000$

$\begin{array}{llll}8 & -2.208220000 & -0.584577000 & -0.168876000\end{array}$

$\begin{array}{llll}1 & -0.935301000 & -0.658975000 & 0.424875000\end{array}$

$\begin{array}{llll}1 & 0.669930000 & -0.846844000 & -1.168945000\end{array}$

$\begin{array}{llll}6 & 1.207835000 & 0.901085000 & -0.118015000\end{array}$

$\begin{array}{llll}6 & 2.290396000 & -1.425401000 & 0.089739000\end{array}$

$\begin{array}{llll}6 & -2.933913000 & 0.590906000 & 0.025487000\end{array}$

$\begin{array}{llll}6 & 1.108092000 & 1.614840000 & 1.073242000\end{array}$

$\begin{array}{llll}6 & 1.462176000 & 1.600569000 & -1.294199000\end{array}$

$\begin{array}{llll}6 & 1.618332000 & 2.980032000 & -1.285169000\end{array}$

$\begin{array}{lll}1.261694000 & 2.994715000 & 1.087746000\end{array}$

$\begin{array}{lll}1.516939000 & 3.682569000 & -0.091715000\end{array}$

$\begin{array}{lll}0.891126000 & 1.085830000 & 1.991071000\end{array}$

$\begin{array}{lll}1.175390000 & 3.534841000 & 2.020884000\end{array}$

$\begin{array}{lll}1.628699000 & 4.757981000 & -0.081668000\end{array}$

$\begin{array}{lll}1.806302000 & 3.507149000 & -2.210631000\end{array}$

$\begin{array}{lll}1.519633000 & 1.060268000 & -2.231205000\end{array}$

$\begin{array}{lll}3.336756000 & -1.075330000 & -0.966565000\end{array}$

$\begin{array}{lll}2.864779000 & -1.171678000 & 1.481609000\end{array}$

$\begin{array}{lll}1.935827000 & -2.907875000 & -0.047203000\end{array}$

$\begin{array}{lll}-2.391297000 & 1.710210000 & -0.871421000\end{array}$

$\begin{array}{llll}6 & -4.403689000 & 0.340844000 & -0.323054000\end{array}$

\section{$\mathrm{TS}_{10}$}

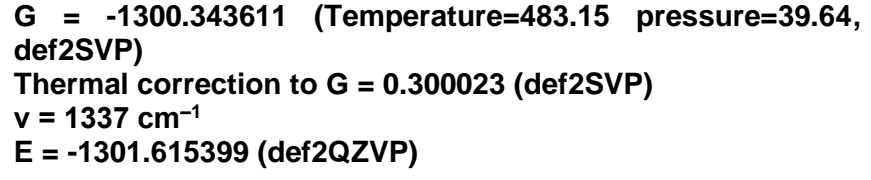

$G=-1300.343611$ (Temperature $=483.15$ pressure=39.64,

$v=1337 \mathrm{~cm}^{-1}$

$\begin{array}{lll}-2.831862000 & 1.038351000 & 1.488200000\end{array}$

$\begin{array}{lll}4.211297000 & -1.716664000 & -0.849748000\end{array}$

$1.488602000-3.112019000 \quad-1.025074000$

$\begin{array}{lll}2.126415000 & -1.373711000 & 2.257129000\end{array}$

$\begin{array}{lll}-4.808977000 & -0.450421000 & 0.310074000\end{array}$

$\begin{array}{lll}-2.417081000 & 1.395334000 & -1.916392000\end{array}$

$\begin{array}{lll}-1.788215000 & 1.225300000 & 1.748614000\end{array}$

$\begin{array}{lll}-3.398440000 & 1.952264000 & 1.676164000\end{array}$

$\begin{array}{lll}-3.212900000 & 0.255971000 & 2.147049000\end{array}$

$\begin{array}{lll}-5.014527000 & 1.235090000 & -0.186079000\end{array}$

$\begin{array}{lll}-4.491113000 & 0.023285000 & -1.363703000\end{array}$

$\begin{array}{lll}-2.973125000 & 2.628971000 & -0.774971000\end{array}$

$\begin{array}{lll}-1.355203000 & 1.933861000 & -0.608789000\end{array}$

$2.946760000-1.222299000-1.975844000$

$3.668429000-0.041316000-0.878233000$

$\begin{array}{lll}2.833009000 & -3.522898000 & 0.028418000\end{array}$

$\begin{array}{lll}1.255358000 & -3.226123000 & 0.742719000\end{array}$

$\begin{array}{lll}3.722629000 & -1.823722000 & 1.653753000\end{array}$

$\begin{array}{lll}3.204352000 & -0.141628000 & 1.590188000\end{array}$

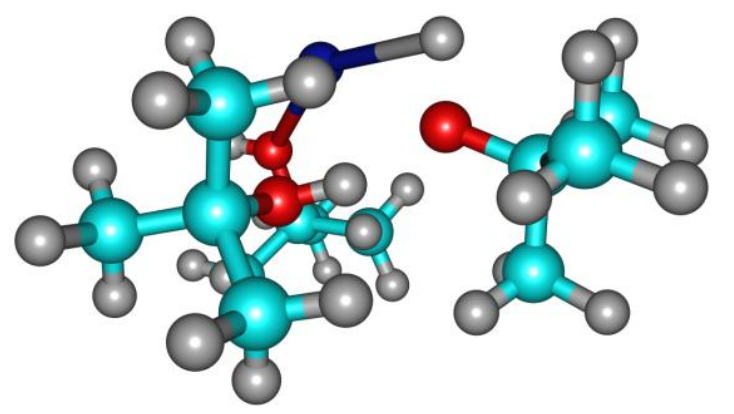

$19 \quad-0.539008000 \quad-0.213444000 \quad-1.957555000$

$8 \quad 1.452984000 \quad-0.986087000-0.468354000$

$1 \quad 1.836940000 \quad-0.922597000-1.646106000$

$\begin{array}{llll}1 & 2.013143000 & -0.820486000 & -2.652053000\end{array}$

$\begin{array}{llll}6 & 2.279866000 & -1.806654000 & 0.323849000\end{array}$

$\begin{array}{llll}6 & 2.252461000 & -3.238719000 & -0.221108000\end{array}$

$\begin{array}{llll}6 & 3.715886000 & -1.269799000 & 0.299776000\end{array}$

$\begin{array}{llll}6 & 1.743520000 & -1.786423000 & 1.755916000\end{array}$

$\begin{array}{llll}1 & 3.745747000 & -0.246157000 & 0.705068000\end{array}$

$\begin{array}{lll}2.631356000 & -3.263903000 & -1.254261000\end{array}$

$\begin{array}{lll}0.728145000 & -2.210880000 & 1.790948000\end{array}$

$\begin{array}{lll}2.384951000 & -2.369984000 & 2.434630000\end{array}$

$\begin{array}{lll}1.695271000 & -0.750451000 & 2.128163000\end{array}$

$\begin{array}{lll}4.393987000 & -1.896506000 & 0.899459000\end{array}$

$\begin{array}{lll}4.095656000 & -1.239873000 & -0.733085000\end{array}$

$\begin{array}{lll}2.870376000 & -3.915498000 & 0.389223000\end{array}$

$\begin{array}{lll}1.219752000 & -3.621738000 & -0.224266000\end{array}$

$\begin{array}{lll}0.870791000 & 0.455501000 & 0.094095000\end{array}$

$\begin{array}{lll}0.273335000 & 1.254642000 & 0.152224000\end{array}$

$\begin{array}{lll}1.066362000 & 2.434788000 & 0.209241000\end{array}$

$\begin{array}{lll}1.995369000 & 2.476732000 & -1.004607000\end{array}$

$\begin{array}{lll}2.619877000 & 1.570139000 & -1.036396000\end{array}$

$\begin{array}{lll}1.411233000 & 2.525340000 & -1.938157000\end{array}$

$\begin{array}{lll}2.657710000 & 3.354770000 & -0.972755000\end{array}$

$\begin{array}{lll}-0.521018000 & 3.583902000 & -0.717230000\end{array}$

$\begin{array}{lll}0.100130000 & 3.612227000 & 0.191308000\end{array}$

$\begin{array}{lll}0.645732000 & 4.566927000 & 0.208947000\end{array}$

$\begin{array}{lll}2.461639000 & 3.358579000 & 1.613391000\end{array}$

$\begin{array}{lll}1.880434000 & 2.431013000 & 1.502605000\end{array}$

$\begin{array}{lll}2.583738000 & 1.583549000 & 1.509316000\end{array}$

$\begin{array}{lll}1.209227000 & 2.331171000 & 2.368969000\end{array}$

$\begin{array}{lll}-0.564858000 & 3.576365000 & 1.067493000\end{array}$

$\begin{array}{lll}-3.701010000 & 0.392313000 & -1.280702000\end{array}$ 
-2.954166000
-3.199635000
-4.489574000
-5.342422000
-4.412476000
-4.698331000
-1.889658000
-2.002863000
-2.138728000
-3.475804000
-3.294026000
-4.121309000
-2.354810000
-1.078499000

$-0.138565000$

$-0.498859000$

$-0.968067000$

0.401406000

$-1.308512000 \quad 0.472940000$

$-0.706946000 \quad 0.120512000$

$-2.205952000 \quad-0.158645000$

$-1.623714000 \quad 1.505445000$

$-2.220355000 \quad 0.186134000$

$-1.336059000 \quad 0.834081000$

$\begin{array}{ll}-1.689207000 & 1.866219000\end{array}$

$0.529036000 \quad 2.297546000$

$0.771109000 \quad 1.240299000$

$1.407255000 \quad 0.887963000$

$1.338854000 \quad 1.167586000$

$-0.739011000$

0.805421000

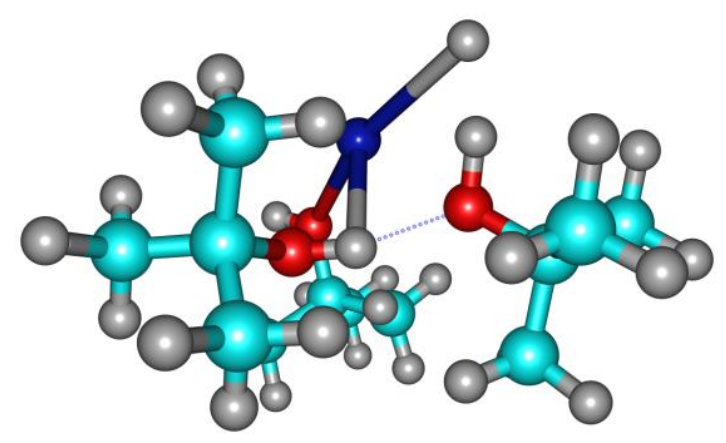

17

$G=-1300.344479$ (Temperature=483.15 pressure=39.64, def2SVP)

Thermal correction to $\mathrm{G}=\mathbf{0 . 3 0 2 5 1}$ (def2SVP)

$E=-1301.619429$ (def2QZVP)

$\begin{array}{lrrr}19 & 0.564686000 & -0.124606000 & 1.981407000 \\ 8 & -1.464453000 & -1.037354000 & 0.474971000 \\ 1 & -1.778520000 & -1.006737000 & 1.456237000 \\ 1 & -1.879847000 & -0.846648000 & 2.858004000 \\ 6 & -2.329298000 & -1.852837000 & -0.311445000 \\ 6 & -2.317327000 & -3.276392000 & 0.242691000 \\ 6 & -3.741641000 & -1.270809000 & -0.267368000 \\ 6 & -1.780038000 & -1.823154000 & -1.732701000 \\ 1 & -3.746068000 & -0.247648000 & -0.674054000 \\ 1 & -2.688555000 & -3.287501000 & 1.278645000 \\ 1 & -0.771364000 & -2.262067000 & -1.765237000 \\ 1 & -2.428448000 & -2.393338000 & -2.414187000 \\ 1 & -1.719315000 & -0.785378000 & -2.097340000 \\ 1 & -4.441126000 & -1.880992000 & -0.858094000 \\ 1 & -4.105564000 & -1.231825000 & 0.770817000 \\ 1 & -2.953989000 & -3.940025000 & -0.361418000 \\ 1 & -1.292302000 & -3.677630000 & 0.237800000 \\ 1 & -0.872336000 & 0.526654000 & -0.143693000 \\ 8 & -0.260837000 & 1.296048000 & -0.161085000 \\ 6 & -1.034167000 & 2.494243000 & -0.233099000 \\ 6 & -1.946475000 & 2.574692000 & 0.989874000 \\ 1 & -2.590833000 & 1.682826000 & 1.045986000 \\ 1 & -1.351580000 & 2.628287000 & 1.915834000 \\ 1 & -2.591303000 & 3.465084000 & 0.948388000 \\ 1 & 0.590151000 & 3.619837000 & 0.655229000 \\ 6 & -0.039737000 & 3.646555000 & -0.247166000 \\ 1 & -0.564342000 & 4.612374000 & -0.275381000 \\ 1 & -2.425578000 & 3.411098000 & -1.640871000 \\ 6 & -1.859385000 & 2.476163000 & -1.517794000 \\ 1 & -2.578456000 & 1.641674000 & -1.499241000 \\ 1 & -1.199289000 & 2.347499000 & -2.388694000 \\ 1 & 0.613667000 & 3.579630000 & -1.129991000 \\ 1 & 3.734793000 & 0.348196000 & 1.250175000 \\ 8 & 2.966036000 & -0.161105000 & 0.955133000 \\ 6 & 3.185192000 & -0.560409000 & -0.407613000 \\ 6 & 4.440650000 & -1.423041000 & -0.471855000 \\ 1 & 5.319856000 & -0.849529000 & -0.138063000 \\ 1 & 4.332125000 & -2.303330000 & 0.178924000 \\ 1 & 4.628129000 & -1.767529000 & -1.499058000 \\ 1 & 1.803115000 & -2.217332000 & -0.138766000\end{array}$

$\begin{array}{rrrr}6 & 1.952046000 & -1.358076000 & -0.812616000 \\ 1 & 2.068740000 & -1.746067000 & -1.834500000 \\ 1 & 3.478776000 & 0.415781000 & -2.328667000 \\ 6 & 3.321991000 & 0.686749000 & -1.274434000 \\ 1 & 4.180025000 & 1.294337000 & -0.946204000 \\ 1 & 2.408536000 & 1.295245000 & -1.201158000 \\ 1 & 1.055035000 & -0.720250000 & -0.799150000\end{array}$

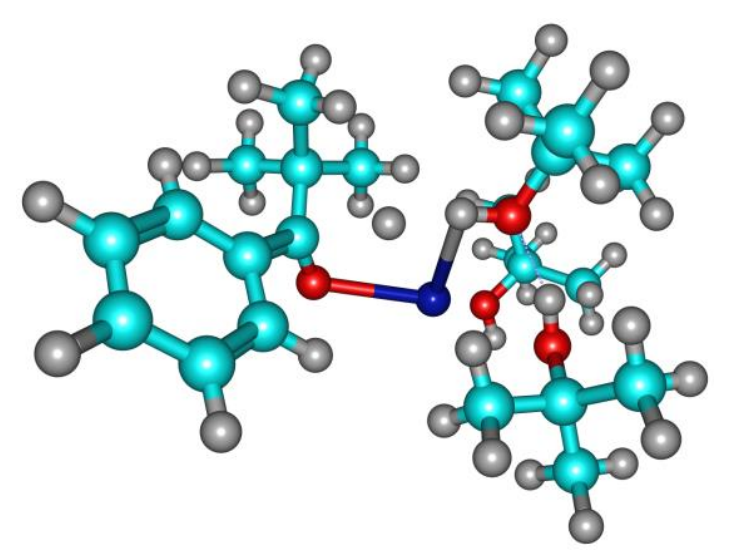

$\mathrm{TS}_{11}$

$G=-1802.40818$ (Temperature $=483.15$ pressure=39.64, def2SVP)

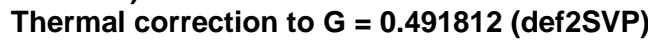

$\mathrm{v}=516 \mathrm{~cm}^{-1}$

$E=-1804.476173$ (def2QZVP)

19

8

1

1

6

6

6

1

1

1

1

1

1

1

8

6

6

1

1

1

6

1

1

6

1
1

1

1

8

6
6

6
1

1

1

1

6
1

1
1
$-0.633679000$ $-0.977903000$ 0.040522000

1.303725000

$-1.274933000$

$-0.931422000$

$-0.463990000$

$-2.769155000$

$-0.702046000$

0.144103000

$-3.349225000$

$-3.078140000$

$-3.016671000$

$-0.681059000$

0.613646000

$-1.179376000$

$-1.493326000$

$-1.867828000$

$-2.220414000$

$-1.762924000$

$-0.244765000$

0.247984000

0.027620000

0.151604000

$-2.207714000$

$-2.459184000$

$-2.150801000$

$-1.849536000$

$-2.147386000$

$-1.648091000$

$-3.235575000$

$-3.550634000$

$-3.391807000$

$-2.885253000$

$-3.811551000$

$-4.594166000$

$-5.166811000$

$-3.907739000$

$-5.303042000$

$-2.235289000$

$-2.960881000$

$-3.595691000$

$-5.429489000$
$-0.228547000$

0.320560000

0.258642000

0.136861000

0.153617000

$-1.272878000$

1.161117000

0.415193000

2.188760000

$-1.465871000$

$-0.302919000$

0.318096000

1.432242000

1.066415000

0.996060000

$-1.435128000$

$-2.001587000$

1.452844000

1.848523000

3.198423000

3.205052000

2.662311000

2.725864000

4.231169000

3.241676000

3.806639000

4.852089000

4.997628000

3.940003000

3.483201000

3.888155000

3.777529000

$-0.943197000$

$-1.292303000$

$-1.801340000$

$-2.953756000$

$-2.604027000$

$-3.747661000$

$-3.381366000$

$-3.042786000$

$-2.286217000$

$-2.744471000$

$-1.018382000$
$-1.244809000$

1.418493000

1.239774000

0.709506000

2.803065000

3.231102000

3.617110000

2.956208000

3.303587000

3.095600000

2.356064000

4.007437000

2.613907000

4.691511000

3.464369000

4.290885000

2.626709000

0.388800000

$-0.439068000$

$-0.526736000$

$-0.714098000$

0.109368000

$-1.669220000$

$-0.733838000$

$-2.646445000$

$-1.735820000$

$-1.879655000$

0.702794000

0.751069000

1.620111000

0.906336000

$-1.600387000$

$-2.869377000$

$-2.121653000$

$-1.149519000$

$-1.769329000$

$-2.642708000$

$-2.099047000$

$-1.045480000$

$-0.321221000$

0.017417000

0.789598000

0.077116000 


$\begin{array}{rrrr}6 & -4.741408000 & -0.674917000 & -0.709352000 \\ 1 & -5.345772000 & -0.320687000 & -1.559845000 \\ 1 & -4.156128000 & 0.174402000 & -0.326063000 \\ 1 & -2.421350000 & -1.447352000 & 0.485380000 \\ 1 & 2.426255000 & -1.254389000 & 2.110489000 \\ 1 & 2.539058000 & -3.032111000 & 2.206299000 \\ 1 & 3.951712000 & -2.089111000 & 1.721319000 \\ 6 & 2.859548000 & -2.146662000 & 1.635565000 \\ 1 & 0.283722000 & -2.029714000 & 0.752798000 \\ 6 & 0.911637000 & -2.749607000 & 0.205048000 \\ 1 & 0.838197000 & -3.720387000 & 0.717989000 \\ 1 & 0.525321000 & -2.883415000 & -0.816302000 \\ 6 & 2.457475000 & -0.986336000 & -0.653463000 \\ 8 & 1.798331000 & -0.926158000 & -1.695221000 \\ 6 & 3.601515000 & -0.000704000 & -0.519596000 \\ 6 & 2.370653000 & -2.278561000 & 0.190137000 \\ 6 & 4.882791000 & -0.340880000 & -0.075114000 \\ 6 & 3.380420000 & 1.303685000 & -0.988557000 \\ 6 & 4.397043000 & 2.249693000 & -0.983866000 \\ 6 & 5.910832000 & 0.608157000 & -0.077768000 \\ 6 & 5.672161000 & 1.903941000 & -0.523357000 \\ 1 & 5.110747000 & -1.350843000 & 0.260651000 \\ 1 & 6.904913000 & 0.321653000 & 0.270450000 \\ 1 & 6.474085000 & 2.644559000 & -0.516791000 \\ 1 & 4.197944000 & 3.263463000 & -1.336605000 \\ 1 & 2.381204000 & 1.571167000 & -1.337235000 \\ 6 & 3.202158000 & -3.336207000 & -0.564651000 \\ 1 & 4.262464000 & -3.058420000 & -0.648751000 \\ 1 & 3.146175000 & -4.297358000 & -0.030960000 \\ 1 & 2.807129000 & -3.480930000 & -1.580923000\end{array}$

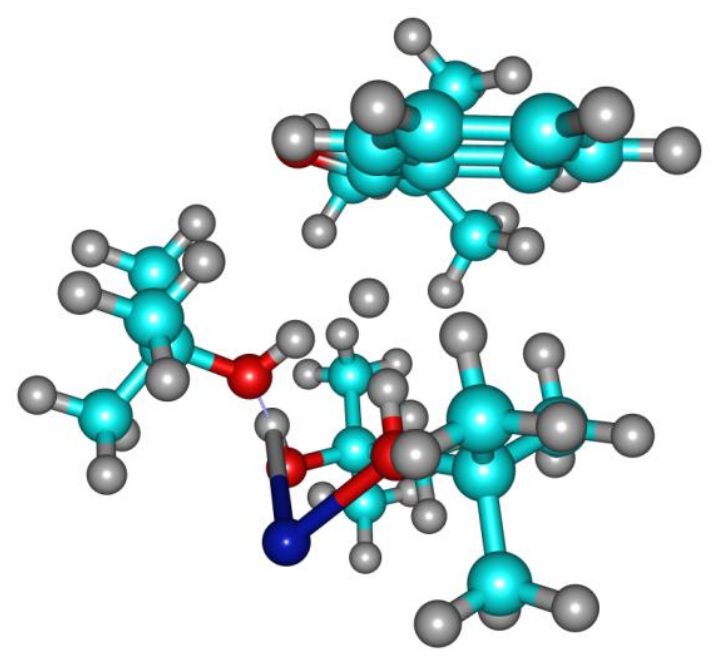

$\mathrm{TS}_{12}$

$G=-1802.399017$ (Temperature $=483.15$ pressure $=39.64$, def2SVP)

Thermal correction to $\mathrm{G}=\mathbf{0 . 4 9 5 1 3 9}$ (def2SVP)

$\mathrm{v}=381 \mathrm{~cm}^{-1}$

$E=-1804.470535$ (def2QZVP)

\begin{tabular}{lr}
1 & 0.694619000 \\
8 & 1.594402000 \\
6 & 1.494374000 \\
6 & 0.244989000 \\
1 & 0.272967000 \\
1 & -0.651045000 \\
1 & 0.163040000 \\
1 & 0.499704000 \\
6 & 1.417296000 \\
1 & 1.398706000 \\
1 & 2.805996000 \\
6 & 2.758323000 \\
1 & 2.778965000 \\
1 & 3.655469000 \\
1 & 2.289233000 \\
8 & 0.260101000 \\
6 & -0.639239000 \\
6 & -1.928656000 \\
1 & -2.372646000 \\
1 & -1.716594000 \\
1 & -2.664073000 \\
1 & 0.297162000 \\
6 & 0.060057000 \\
1 & -0.580035000 \\
1 & -1.620126000 \\
6 & -0.930723000 \\
1 & -1.393539000 \\
1 & -0.000040000 \\
1 & 0.995534000 \\
1 & -0.508838000 \\
1 & 3.090792000 \\
6 & -1.922463000 \\
8 & -1.749474000 \\
6 & -3.024463000 \\
6 & -1.438938000 \\
6 & -3.627188000 \\
6 & -3.517104000 \\
6 & -4.549451000 \\
6 & -4.665321000 \\
6 & -5.128823000 \\
1 & -3.313622000 \\
1 & -5.117410000 \\
1 & -5.939930000 \\
1 & -3.0 .79 \\
1 & -1.907173000 \\
1 & -3.073325000 \\
6 & -2.639602000 \\
6 & -0.899813000 \\
6 & -0.314744000 \\
1 & -3.440044000 \\
1 & -0.666108000 \\
1 & -2.092508000 \\
\hline
\end{tabular}

$-0.501186000$

$-0.475806000$

$-1.059792000$

$-0.520092000$

0.580110000

$-0.824300000$

$-0.914095000$

$-2.859155000$

$-2.578691000$

$-3.057250000$

$-1.143588000$

$-0.650714000$

0.436557000

$-0.936523000$

$-2.958900000$

2.085126000

3.003538000

3.047946000

2.041685000

3.372149000

3.737184000

4.663522000

4.357325000

5.131327000

3.243933000

2.549323000

1.550228000

2.493772000

4.309872000

$-0.314381000$

$-0.554968000$

$-1.730713000$

$-2.352454000$

$-0.674501000$

$-2.393550000$

$-0.101058000$

$-0.309242000$

0.614417000

0.823887000

1.190461000

$-0.374076000$

1.254131000

1.914118000

0.883571000

$-0.772798000$

$-3.206451000$

$-1.470706000$

$-3.366014000$

$-2.567192000$

$-4.168805000$

$-1.088034000$

$-3.872006000$

$-3.832713000$

$-3.818771000$

$-2.825039000$

$-2.049604000$

$-0.603271000$
0.767802000

1.251374000

2.553296000

3.244004000

3.294158000

2.680576000

4.268158000

1.886490000

2.424042000

3.414683000

4.284979000

3.303388000

3.487856000

2.731779000

1.868974000

0.165591000

$-0.447684000$

0.368480000

0.408668000

1.398996000

$-0.073767000$

0.588542000

$-0.442525000$

$-0.890487000$

$-2.380137000$

$-1.876816000$

$-1.862929000$

$-2.462739000$

$-1.023090000$

0.030200000

0.251937000

0.031026000

1.078808000

0.044898000

$-1.286282000$

$-1.084161000$

1.307884000

1.444493000

$-0.949724000$

0.312208000

$-2.088876000$

$-1.845628000$

0.413325000

2.440401000

2.189364000

$-1.813906000$

$-2.384791000$

$-0.923438000$

$-2.212891000$

$-0.261484000$

$-2.111869000$

$-2.624541000$

$-1.015485000$

$-1.838623000$

$-0.405276000$

$-3.317218000$

$-2.602309000$
$19 \quad 2.674705000$

$6 \quad 3.661589000$

$8 \quad 3.862367000$

3.281103000

2.336445000

3.146917000

4.064926000

4.981963000

4.902357000

5.269399000

5.778302000

2.546984000

2.856456000

2.300436000

1.630539000
1.158551000

1.965371000 $-0.319401000$

$-0.159267000$

$-1.770135000$

$-2.019563000$

$-1.945394000$

$-2.446638000$

0.037119000

$-0.049332000$

1.070860000

$-0.636485000$

0.622550000

1.676535000

0.462559000

0.457521000
0.109531000

0.961789000

$-1.615323000$

$-0.207461000$

$-1.896077000$

$-1.387550000$

$-2.973579000$

$-1.523510000$

$-2.281689000$

$-3.374836000$

$-2.035702000$

$-1.932131000$

$-2.075346000$

$-1.967587000$

$-3.135720000$

$-1.484699000$

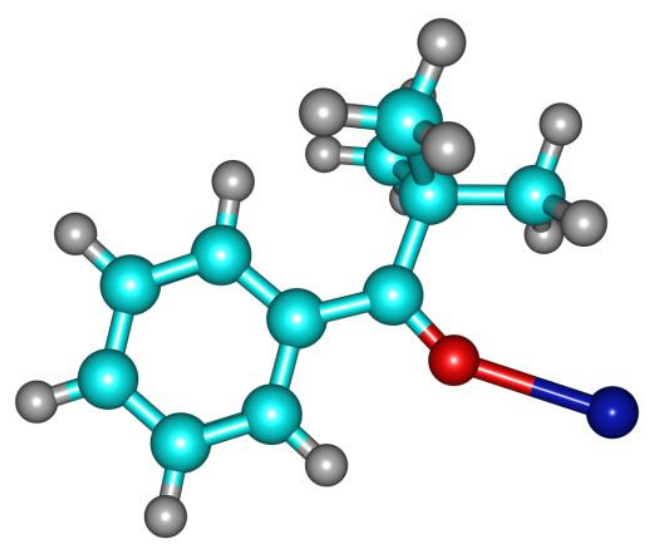


20

$\begin{array}{lccc}\mathbf{G}=\mathbf{- 1 1 0 2 . 6 6 1 6 9 2}(\text { Temperature }=\mathbf{4 8 3 . 1 5}, \text { pressure }=\mathbf{3 9 . 6 4 )} \\ \mathbf{E}=\mathbf{- 1 1 0 2 . 8 0 5 7 3 1 1 2} & & \\ 6 & -0.237616000 & 0.082400000 & -0.000212000 \\ 8 & -1.074526000 & -0.897065000 & -0.000350000 \\ 19 & -3.329343000 & -1.751375000 & 0.000265000 \\ 6 & -0.821408000 & 1.509161000 & -0.000098000 \\ 6 & 1.164410000 & -0.194846000 & -0.000109000 \\ 6 & 2.217818000 & 0.770552000 & 0.000287000 \\ 6 & 1.588777000 & -1.561611000 & -0.000371000 \\ 6 & 3.545247000 & 0.395947000 & 0.000417000 \\ 6 & 2.919626000 & -1.916305000 & -0.000253000 \\ 6 & 3.928616000 & -0.948450000 & 0.000152000 \\ 1 & 1.997326000 & 1.824781000 & 0.000518000 \\ 1 & 0.830464000 & -2.330151000 & -0.000687000 \\ 1 & 4.303964000 & 1.168774000 & 0.000743000 \\ 1 & 3.184759000 & -2.966617000 & -0.000477000 \\ 1 & 4.971839000 & -1.229569000 & 0.000255000 \\ 6 & -0.416253000 & 2.281931000 & 1.264529000 \\ 1 & 0.657060000 & 2.426085000 & 1.351643000 \\ 1 & -0.885237000 & 3.268281000 & 1.266674000 \\ 1 & -0.751357000 & 1.749205000 & 2.155855000 \\ 6 & -2.351212000 & 1.433009000 & -0.000374000 \\ 1 & -2.721652000 & 0.925479000 & -0.892100000 \\ 1 & -2.721969000 & 0.925519000 & 0.891248000 \\ 1 & -2.766821000 & 2.441230000 & -0.000458000 \\ 6 & -0.415808000 & 2.282315000 & -1.264326000 \\ 1 & 0.657595000 & 2.425825000 & -1.351399000 \\ 1 & -0.751253000 & 1.750230000 & -2.155909000 \\ 1 & -0.884182000 & 3.268958000 & -1.265985000\end{array}$

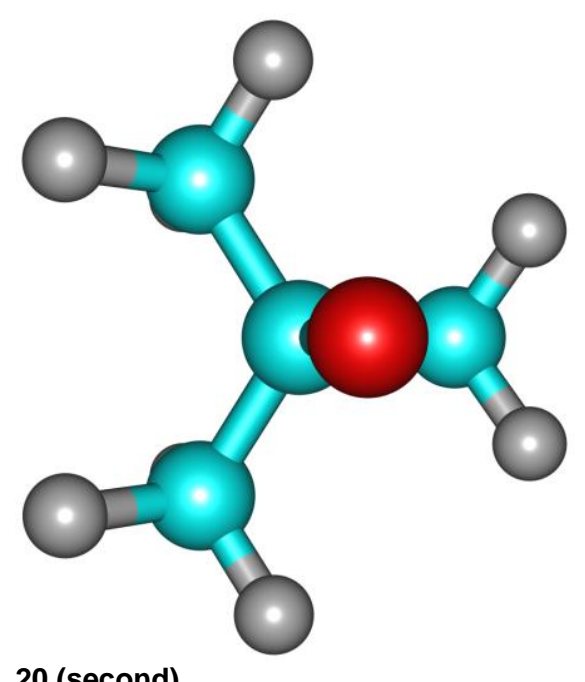

20 (second)

$\mathrm{G}=\mathbf{- 2 3 2 . 9 3 3 3 6 5}($ Temperature $=483.15$, pressure $=39.64)$

$E=-\mathbf{2 3 3 . 0 0 7 4 9 4 7 6 3}$

$\begin{array}{lcrr}8 & 0.000049000 & 0.269777000 & 1.424869000 \\ 6 & -0.000005000 & -0.026613000 & 0.074580000 \\ 6 & 1.265223000 & -0.788621000 & -0.308431000 \\ 1 & 1.294380000 & -1.748254000 & 0.206937000 \\ 1 & 1.285263000 & -0.974566000 & -1.381501000 \\ 1 & 2.150029000 & -0.216604000 & -0.033308000 \\ 6 & 0.000159000 & 1.374219000 & -0.580486000 \\ 1 & -0.888462000 & 1.931896000 & -0.293211000 \\ 1 & 0.888908000 & 1.931691000 & -0.293211000 \\ 1 & 0.000141000 & 1.231929000 & -1.660374000 \\ 6 & -1.265418000 & -0.788319000 & -0.308411000 \\ 1 & -1.294787000 & -1.747955000 & 0.206940000 \\ 1 & -2.150084000 & -0.216101000 & -0.033253000 \\ 1 & -1.285534000 & -0.974240000 & -1.381484000\end{array}$

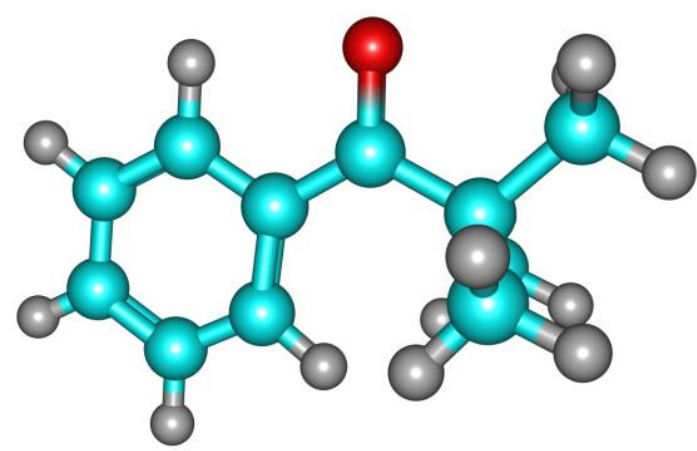

21

$\mathrm{G}=-\mathbf{5 0 2 . 7 7 5 5 3 6}($ Temperature $=483.15$, pressure $=39.64)$ $E=-502.927269541$

$6-0.694576000$

$0.740223000-0.000200000$

$\begin{array}{lll}0.64626000 & 1.991792000 & 0.000266000\end{array}$

$\begin{array}{llll}6 & -1.897730000 & -0.230687000 & 0.000094000\end{array}$

$\begin{array}{llll}6 & 0.664376000 & 0.285241000 & 0.000032000\end{array}$

$\begin{array}{llll}6 & 1.111421000 & -1.075990000 & -0.000244000\end{array}$

$\begin{array}{llll}6 & 1.713104000 & 1.264694000 & 0.000132000\end{array}$

$\begin{array}{llll}6 & 2.450483000 & -1.403373000 & -0.000392000\end{array}$

$\begin{array}{llll}6 & 3.044716000 & 0.917939000 & -0.000013000\end{array}$

$\begin{array}{llll}6 & 3.450335000 & -0.422915000 & -0.000278000\end{array}$

$\begin{array}{llll}1 & 0.403140000 & -1.887567000 & -0.000347000\end{array}$

$\begin{array}{llll}1 & 1.433902000 & 2.307957000 & 0.000335000\end{array}$

$\begin{array}{llll}1 & 2.728784000 & -2.450472000 & -0.000604000\end{array}$

$\begin{array}{lll}3.791625000 & 1.703127000 & 0.000082000\end{array}$

$\begin{array}{lll}4.496797000 & -0.691861000 & -0.000396000\end{array}$

$\begin{array}{lll}-1.913433000 & -1.101482000 & -1.265432000\end{array}$

$\begin{array}{lll}-1.038507000 & -1.738604000 & -1.361973000\end{array}$

$\begin{array}{lll}-2.795913000 & -1.745750000 & -1.266624000\end{array}$

$\begin{array}{lll}-1.960342000 & -0.469701000 & -2.154003000\end{array}$

$\begin{array}{lll}-3.198824000 & 0.574961000 & 0.000382000\end{array}$

$\begin{array}{lll}-3.274400000 & 1.212706000 & 0.879930000\end{array}$

$\begin{array}{lll}-3.274585000 & 1.213037000 & -0.878910000\end{array}$

$\begin{array}{lll}-4.048395000 & -0.110515000 & 0.000341000\end{array}$

$-1.913205000 \quad-1.102037000 \quad 1.265236000$

$\begin{array}{lll}-1.038211000 & -1.739124000 & 1.361384000\end{array}$

$\begin{array}{lll}-1.960052000 & -0.470648000 & 2.154091000\end{array}$

$\begin{array}{lll}-2.795634000 & -1.746374000 & 1.266259000\end{array}$

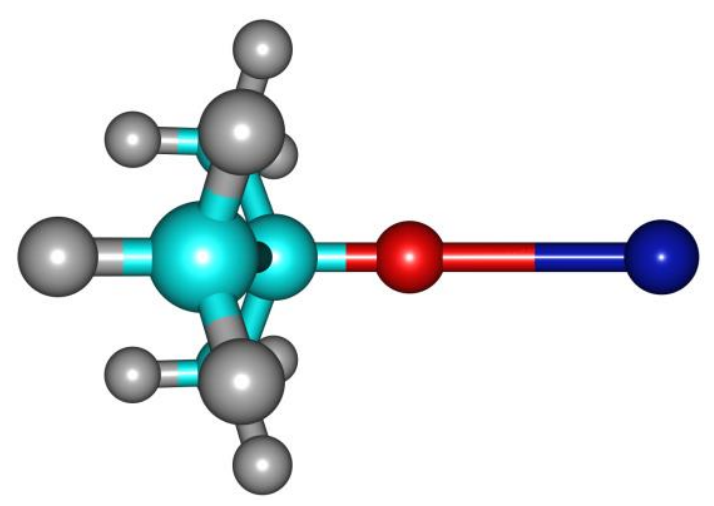

21 (second)

$\mathrm{G}=-\mathbf{8 3 2 . 7 9 9 4 8 1}$ (Temperature $=483.15$, pressure $=39.64$ )

$E=-832.861733233$

$\begin{array}{llll}19 & -2.722930000 & -0.000071000 & 0.033035000\end{array}$

$\begin{array}{llll}8 & -0.178154000 & -0.000468000 & 0.064602000\end{array}$

$\begin{array}{lllll}6 & 1.193515000 & -0.000095000 & 0.039695000\end{array}$

$\begin{array}{llll}6 & 1.485865000 & 0.001862000 & -1.491449000\end{array}$

$\begin{array}{llll}6 & 1.751027000 & 1.268901000 & 0.675232000\end{array}$

$\begin{array}{llll}6 & 1.751711000 & -1.270185000 & 0.672345000\end{array}$

$\begin{array}{lll}1.497194000 & 1.297228000 & 1.734177000\end{array}$

$\begin{array}{lll}1.076194000 & 0.893488000 & -1.958850000\end{array}$

$\begin{array}{lll}1.340139000 & -2.151744000 & 0.183862000\end{array}$

$\begin{array}{lll}2.836047000 & -1.288155000 & 0.577759000\end{array}$

$\begin{array}{lll}1.497842000 & -1.301004000 & 1.731224000\end{array}$

$\begin{array}{lll}2.835349000 & 1.287659000 & 0.580605000\end{array}$

$\begin{array}{lll}1.338953000 & 2.151285000 & 0.188677000\end{array}$

$\begin{array}{lll}2.569858000 & 0.002238000 & -1.595818000\end{array}$ 


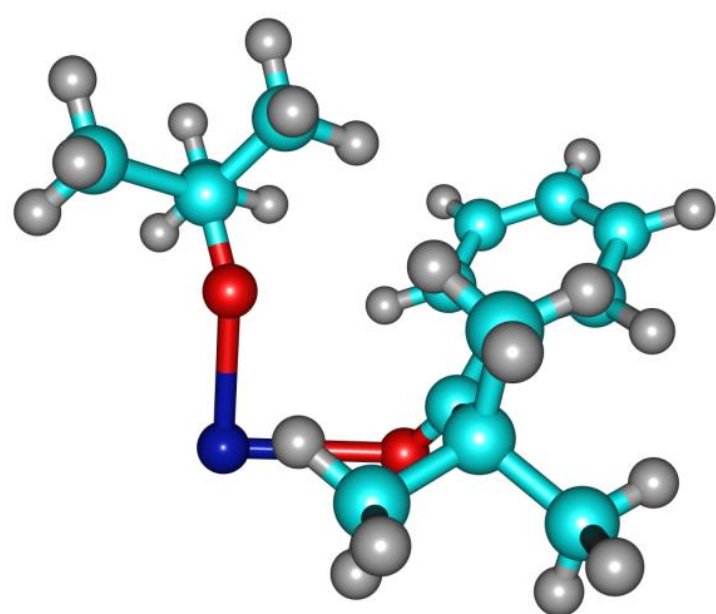

22

$G=-1335.666293($ Temperature $=483.15$, pressure $=39.64)$

\section{$E=-1335.91768589$}

$6-1220416000$

$8 \quad 0.696016000$

$6-2.680789000$

$8-2.096083000$

$19-1.878989000$

$-4.018426000$

$-3.850129000$

$-4.503935000$

$-4.703577000$

$-2.953525000$

$-3.442619000$

$-2.013254000$

$-3.595343000$

$-1.768662000$

$-0.801831000$

$-2.194067000$

$-1.596228000$

1.308298000

1.756164000

2.937829000

1.025056000

3.381855000

1.454634000

2.638016000

3.530822000

0.105493000

4.309002000

0.868165000

2.980512000

1.227379000

2.108427000

0.351302000

1.130584000

0.142492000

0.156341000

0.221829000

$-0.806303000$

2.627645000

2.694621000

2.658646000

3.502583000
$-0.936736000$

0.973252000

$-0.173378000$

$-1.600046000$

$-0.068688000$

1.530598000

0.190036000

1.973706000

2.886833000

2.254638000

1.510216000

1.657448000

1.884972000

2.587155000

0.986677000

$-1.899458000$

0.458994000

0.808134000

1.444491000

2.122095000

2.760985000

3.100808000

0.061849000

1.170708000

2.381128000

3.520316000

4.126183000

$-1.174964000$

$-0.575761000$

$-0.526489000$

$-1.916702000$

$-2.887112000$

$-3.440703000$

$-3.606897000$

$-2.358350000$

$-2.677903000$

$-3.394116000$

$-3.228984000$

$-2.032612000$
$-1.297074000$

0.646674000
$-0.464068000$

0.684047000

0.189073000

$-1.736722000$

1.370433000

2.177955000

1.791069000

0.653003000

$-0.453023000$

$-0.104866000$

$-0.932215000$

$-1.205863000$

1.716226000

1.261539000

2. 101769000

2.560376000

0.720045000

$-0.398501000$

0.251988000

$-1.060961000$

0.229294000

$-1.057968000$

$-0.415404000$

0.757708000

$-1.558216000$

0.720665000

$-1.556114000$

$-0.417324000$

2.065447000

2.099217000

2.858349000

0.631012000

$-0.306583000$

1.444770000

0.741818000

0.589799000

1.409183000

$-0.350089000$

0.636079000
$-1.503237000$

2.278621000

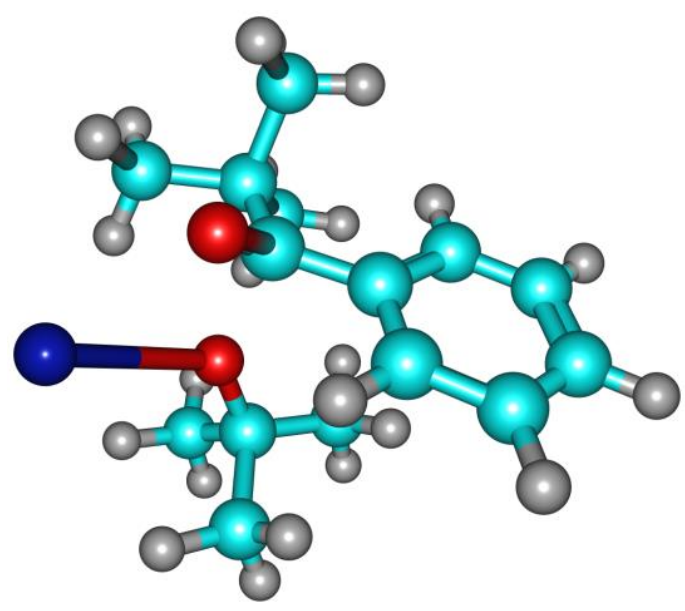

$\mathrm{TS}_{13}$

$\mathrm{G}=\mathbf{- 1 3 3 5 . 6 4 6 0 1 5}$ (Temperature $=483.15$, pressure $=39.64$ )

$v=160 \mathrm{~cm}^{-1}$

$E=-1335.90491674$

0.607184000

0.651400000

0.903730000

0.985398000

$-0.471573000$

$\begin{array}{llll}8 & 0.959070000 & -0.659468000 & 0.178129000\end{array}$

$\begin{array}{llll}19 & 2.633136000 & -0.515577000 & -1.685558000\end{array}$

$\begin{array}{lll}1.797291000 & -1.991194000 & 1.966694000\end{array}$

$\begin{array}{lll}1.861698000 & -1.152930000 & 2.661316000\end{array}$

$\begin{array}{lll}1.666151000 & -2.905361000 & 2.548881000\end{array}$

$\begin{array}{lll}2.747426000 & -2.061513000 & 1.432697000\end{array}$

$\begin{array}{lll}0.569706000 & -3.018371000 & 0.060540000\end{array}$

$\begin{array}{lll}0.355329000 & -3.925775000 & 0.628599000\end{array}$

$\begin{array}{lll}-0.217039000 & -2.887392000 & -0.682898000\end{array}$

$\begin{array}{lll}1.517533000 & -3.172681000 & -0.461503000\end{array}$

$\begin{array}{lll}-0.663464000 & -1.685250000 & 1.752554000\end{array}$

$\begin{array}{lll}-1.530247000 & -1.692311000 & 1.093163000\end{array}$

$\begin{array}{lll}-0.749673000 & -2.540283000 & 2.425183000\end{array}$

$\begin{array}{lll}-0.697975000 & -0.779283000 & 2.355175000\end{array}$

$\begin{array}{lll}0.671585000 & 1.948425000 & 0.540406000\end{array}$

$\begin{array}{lll}-1.331139000 & 0.453243000 & -0.433421000\end{array}$

$\begin{array}{lll}-2.326648000 & 0.971879000 & 0.386631000\end{array}$

$\begin{array}{lll}-1.688270000 & -0.540216000 & -1.347089000\end{array}$

$\begin{array}{lll}-3.627773000 & 0.479275000 & 0.328343000\end{array}$

$\begin{array}{lll}-2.976935000 & -1.038638000 & -1.405208000\end{array}$

$\begin{array}{lll}-3.956783000 & -0.534724000 & -0.555102000\end{array}$

$\begin{array}{lll}-2.118660000 & 1.765040000 & 1.084331000\end{array}$

$\begin{array}{lll}-0.924304000 & -0.931793000 & -2.003069000\end{array}$

$\begin{array}{lll}-4.382815000 & 0.896884000 & 0.980455000\end{array}$

$\begin{array}{lll}-3.221063000 & -1.822302000 & -2.109745000\end{array}$

$\begin{array}{lll}-4.965704000 & -0.921796000 & -0.592488000\end{array}$

$\begin{array}{lll}0.422796000 & 1.667090000 & 2.020625000\end{array}$

$\begin{array}{lll}-0.625202000 & 1.568272000 & 2.286277000\end{array}$

$\begin{array}{lll}0.933872000 & 0.757493000 & 2.323977000\end{array}$

$\begin{array}{lll}0.827943000 & 2.493686000 & 2.607624000\end{array}$

$\begin{array}{lll}2.188779000 & 2.063036000 & 0.358819000\end{array}$

$\begin{array}{lll}2.451790000 & 2.312238000 & -0.668001000\end{array}$

$\begin{array}{lll}2.570514000 & 2.858085000 & 1.000316000\end{array}$

$\begin{array}{lll}2.682008000 & 1.136656000 & 0.653677000\end{array}$

$0.062659000 \quad 3.306911000 \quad 0.151178000$

$0.487946000 \quad 4.089475000 \quad 0.781059000$

$\begin{array}{lll}0.291951000 & 3.545969000 & -0.886842000\end{array}$

$\begin{array}{lll}-1.018499000 & 3.337321000 & 0.270651000\end{array}$ 


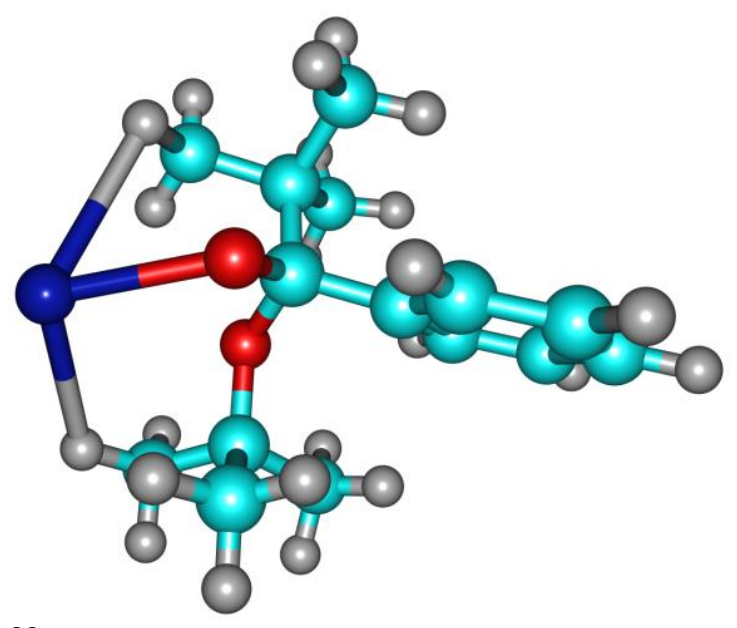

23

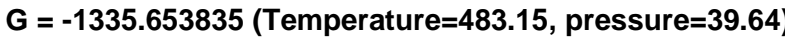

$E=-\mathbf{1 3 3 5 . 9 1 6 4 8 9 2 9}$

$\begin{array}{lrrr}6 & 0.115442000 & 0.530320000 & -0.187598000 \\ 8 & 0.658044000 & 0.672674000 & -1.387662000 \\ 6 & 0.981496000 & -1.846530000 & 0.433774000 \\ 8 & 0.903872000 & -0.427789000 & 0.653031000 \\ 19 & 3.066677000 & 0.292601000 & -1.083626000 \\ 6 & 2.367776000 & -2.247168000 & 0.943287000 \\ 1 & 2.507967000 & -1.896523000 & 1.965420000 \\ 1 & 2.478875000 & -3.330464000 & 0.931621000 \\ 1 & 3.180905000 & -1.849063000 & 0.334741000 \\ 6 & 0.816935000 & -2.282910000 & -1.020997000 \\ 1 & 0.879661000 & -3.371074000 & -1.060809000 \\ 1 & -0.147725000 & -1.980489000 & -1.421027000 \\ 1 & 1.586735000 & -1.885836000 & -1.681041000 \\ 6 & -0.040341000 & -2.603769000 & 1.288744000 \\ 1 & -1.043215000 & -2.543667000 & 0.877058000 \\ 1 & 0.235657000 & -3.657221000 & 1.336901000 \\ 1 & -0.050227000 & -2.207534000 & 2.304341000 \\ 6 & 0.217589000 & 1.881792000 & 0.657656000 \\ 6 & -1.364631000 & 0.082586000 & -0.255202000 \\ 6 & -2.100516000 & -0.323946000 & 0.856360000 \\ 6 & -2.027004000 & 0.156604000 & -1.477759000 \\ 6 & -3.448044000 & -0.642798000 & 0.752222000 \\ 6 & -3.374249000 & -0.163634000 & -1.592150000 \\ 6 & -4.093915000 & -0.561856000 & -0.474498000 \\ 1 & -1.615135000 & -0.419140000 & 1.815113000 \\ 1 & -1.465692000 & 0.469186000 & -2.346169000 \\ 1 & -3.992526000 & -0.961025000 & 1.631324000 \\ 1 & -3.862159000 & -0.098100000 & -2.555565000 \\ 1 & -5.143508000 & -0.808993000 & -0.557144000 \\ 6 & -0.231625000 & 1.783829000 & 2.118130000 \\ 1 & -1.309739000 & 1.673237000 & 2.209758000 \\ 1 & 0.250053000 & 0.957054000 & 2.638723000 \\ 1 & 0.036202000 & 2.706406000 & 2.637275000 \\ 6 & 1.670486000 & 2.365009000 & 0.656827000 \\ 1 & 2.013468000 & 2.570889000 & -0.357192000 \\ 1 & 1.748266000 & 3.300759000 & 1.210927000 \\ 1 & 2.331166000 & 1.649645000 & 1.151488000 \\ 6 & -0.630824000 & 2.951645000 & -0.031739000 \\ 1 & -0.490372000 & 3.913979000 & 0.464212000 \\ 1 & -0.349017000 & 3.064143000 & -1.078070000 \\ 1 & -1.693287000 & 2.710280000 & 0.011703000\end{array}$

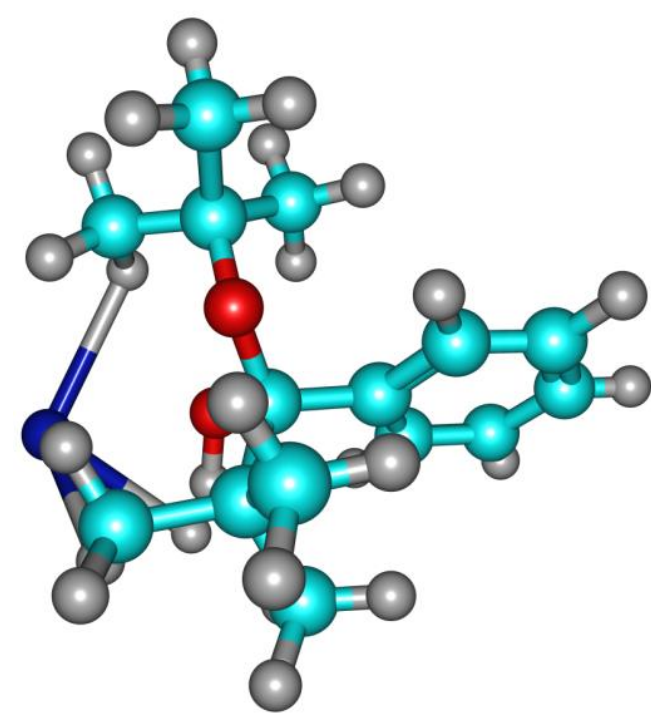

$\mathrm{TS}_{14}$

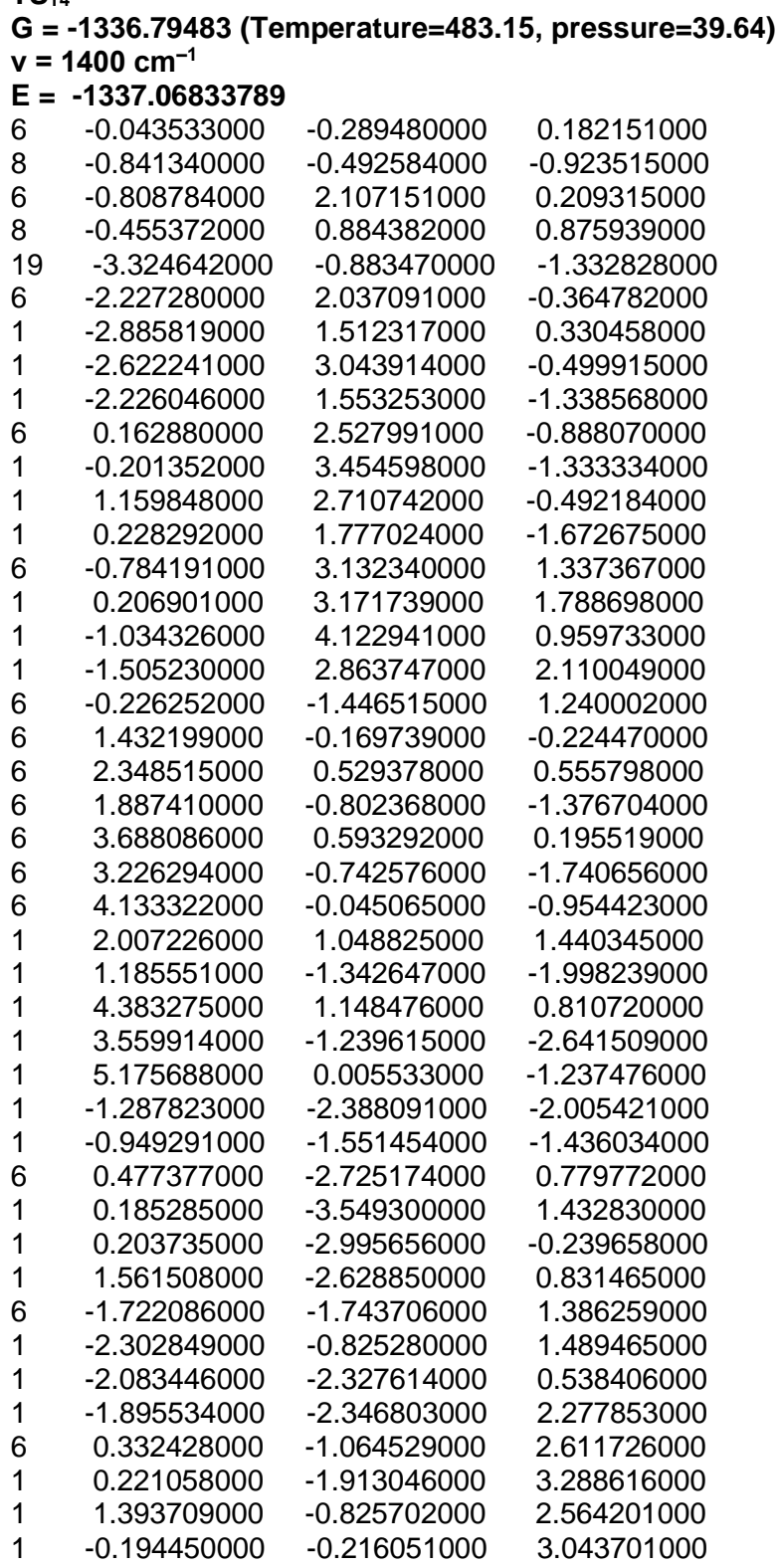




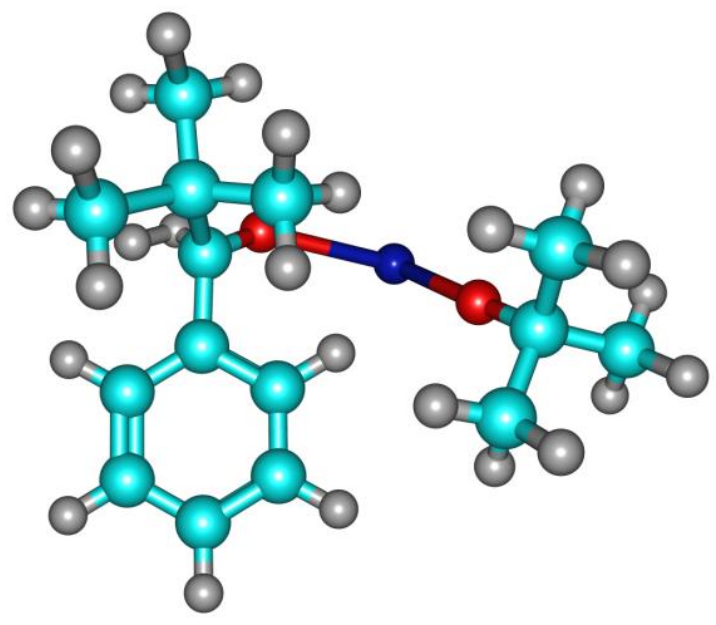

$\mathrm{TS}_{15}$

$\mathrm{G}=\mathbf{- 1 3 3 6 . 7 3 3 9 2}($ Temperature $=483.15$, pressure $=39.64)$

$v=1698 \mathrm{~cm}^{-1}$

$E=-1336.99964361$

$6-1.917388000$

$8-1.164844000$

3.320010000

$-0.804089000$

0.486137000

$-1.254547000$

1.531679000

2.534841000

0.186441000

$-0.596558000$

0.480270000

$1.192091000-0.807615000$

4.759842000

5.190873000

5.408412000

4.754823000

2.770257000

3.371144000

1.749452000

2.748729000

3.359328000

2.351484000

4.002290000

3.726761000

$-1.883581000$

$-1.886354000$

$-0.639930000$

$-3.041305000$

$-0.559590000$

$-2.957070000$

$-1.715676000$

0.270299000

$-4.012072000$

0.410591000

$-3.861320000$

$-1.648210000$

$-3.186388000$

$-2.627566000$

$-0.481984000$

$-0.362992000$

$-0.322756000$

0.290826000

$-2.131395000$

$-3.094296000$

$-2.148058000$

$-1.352100000$

$-2.937570000$

$-3.942333000$

$-2.755524000$

$-2.917338000$
0.479829000

$-0.411212000$

0.786220000

1.276644000

1.444789000

1.744635000

1.263894000

2.276811000

$-0.948380000$

$-1.141731000$

$-0.717813000$

$-1.863578000$

$-1.677160000$

0.695791000

1.310861000

1.459325000

2.682967000

2.833487000

3.447118000

0.722941000

0.984045000

3.155113000

3.421807000

4.517010000

$-0.957371000$

$-1.225626000$

$-2.272935000$

$-0.549461000$

$-1.718829000$

$-3.130285000$

$-3.240874000$

$-3.754340000$

$-3.498420000$

$-1.227737000$

$-1.270566000$

$-0.212779000$

$-1.891436000$
$-1.538438000$
2.358923000

$-0.142932000$

0.318541000

$-0.967135000$

0.603624000

$-1.290923000$

$-2.152769000$

$-1.634215000$

$-0.584150000$

$-1.634799000$

$-2.009176000$

$-2.487621000$

$-1.166015000$

$-0.786834000$

0.285844000

0.205438000

0.182439000

0.015916000

0.002778000

$-0.082411000$

0.271466000

0.251935000

$-0.052236000$

$-0.066005000$

$-0.221654000$

0.778507000

1.465619000

$-1.386416000$

$-2.183060000$

$-0.638218000$

$-0.389837000$

0.115730000

$-1.282484000$

0.273093000

$-1.794910000$

$-1.367818000$

$-2.145444000$

$-2.658916000$
$-1.814326000$

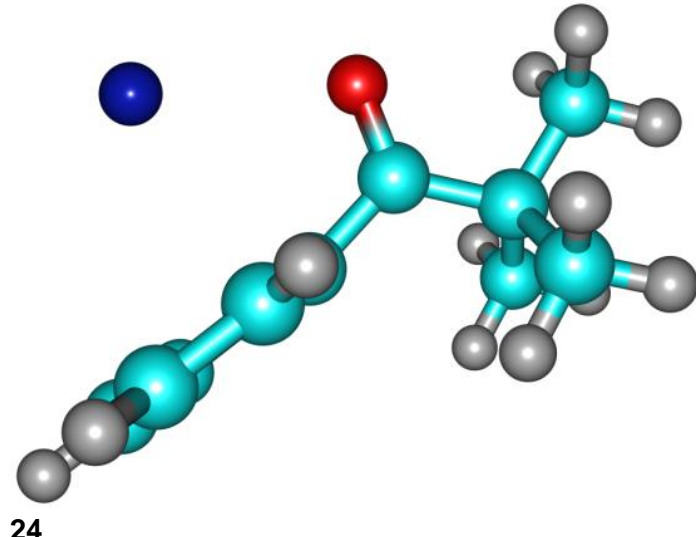

24

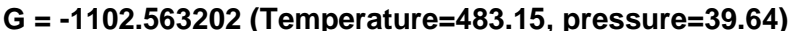

$E=-1102.70964333$

$\begin{array}{llll}6 & -0.951732000 & 0.441065000 & 0.001514000\end{array}$

$\begin{array}{llll}8 & -0.774776000 & 1.645420000 & 0.002234000\end{array}$

$\begin{array}{llll}19 & 1.896688000 & 2.082856000 & -0.000257000\end{array}$

$\begin{array}{llll}6 & -2.322428000 & -0.192914000 & 0.000552000\end{array}$

$\begin{array}{llll}6 & 0.287882000 & -0.413683000 & 0.000388000\end{array}$

$\begin{array}{llll}6 & 0.914027000 & -0.714634000 & 1.207947000\end{array}$

$\begin{array}{llll}6 & 0.913404000 & -0.714905000 & -1.207299000\end{array}$

$\begin{array}{llll}6 & 2.171575000 & -1.305361000 & 1.203575000\end{array}$

$\begin{array}{llll}6 & 2.170953000 & -1.305824000 & -1.203254000\end{array}$

$\begin{array}{llll}6 & 2.802519000 & -1.594636000 & 0.000018000\end{array}$

$\begin{array}{llll}1 & 0.427570000 & -0.473836000 & 2.144138000\end{array}$

$\begin{array}{llll}1 & 0.426799000 & -0.474014000 & -2.143370000\end{array}$

$\begin{array}{llll}1 & 2.658952000 & -1.533938000 & 2.140639000\end{array}$

$\begin{array}{llll}1 & 2.657862000 & -1.534825000 & -2.140458000\end{array}$

$\begin{array}{llll}1 & 3.783632000 & -2.047316000 & -0.000170000\end{array}$

$\begin{array}{llll}6 & -2.443004000 & -1.085516000 & 1.243867000\end{array}$

$\begin{array}{llll}1 & -3.435928000 & -1.534176000 & 1.252271000\end{array}$

$\begin{array}{llll}1 & -1.709831000 & -1.890732000 & 1.237278000\end{array}$

$\begin{array}{llll}1 & -2.323754000 & -0.508452000 & 2.160942000\end{array}$

$\begin{array}{llll}6 & -3.397165000 & 0.885421000 & 0.013914000\end{array}$

$\begin{array}{llll}1 & -3.324034000 & 1.527903000 & -0.862467000\end{array}$

$\begin{array}{llll}1 & -4.379060000 & 0.413984000 & 0.012421000\end{array}$

$\begin{array}{llll}1 & -3.318559000 & 1.511025000 & 0.902006000\end{array}$

$6 \quad-2.448238000 \quad-1.058923000 \quad-1.261284000$

$\begin{array}{llll}1 & -2.332233000 & -0.462457000 & -2.166273000\end{array}$

$\begin{array}{llll}1 & -1.715555000 & -1.864503000 & -1.274447000\end{array}$

$\begin{array}{llll}1 & -3.441472000 & -1.506824000 & -1.275123000\end{array}$

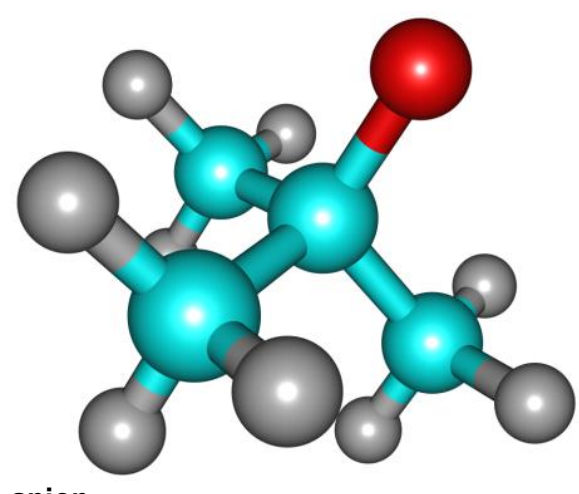

anion

$\mathrm{G}=\mathbf{- 2 3 3 . 0 9 2 7 6 3}($ Temperature $=483.15$, pressure $=39.64)$

$E=-233.168150104$

$6-0.000628000$

$-0.009137000$

1.098004000

0.000160000

0.121219000

$0.000310000 \quad 1.496279000$

$0.933332000-0.424485000$

2.074102000

0.602671000

$-0.063353000$

1.128916000

0.963642000

$-1.516906000$

0.931955000

$-1.354482000$

1.948615000

0.480735000

$-0.058149000$

$-0.435750000$

$-1.389468000$

0.489214000

$-1.528405000$

$-0.170817000 \quad-0.072045000$

$-1.558873000$

1.492325000

$-0.078643000$

0.264270000

$-1.414434000$

$-0.427923000$ 


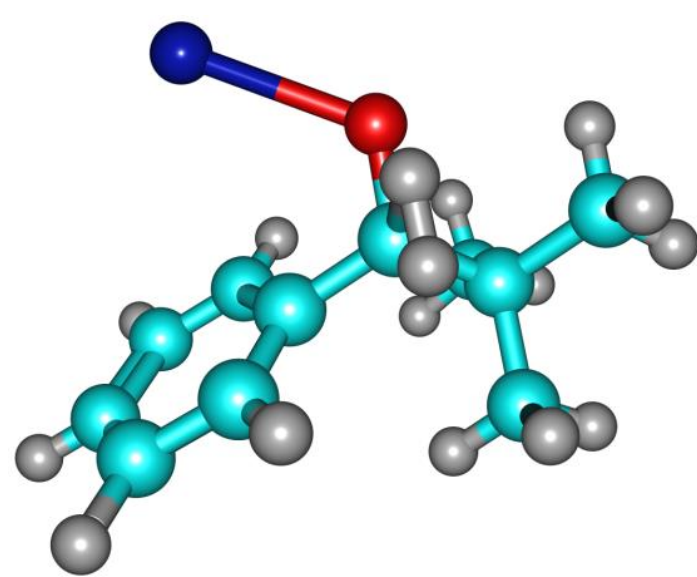

$-0.744799000$

$-1.096855000$

$-2.467053000$

$-2.116854000$

$-2.977249000$

$-0.073901000$

$-2.514764000$

$-4.046857000$

$-3.136969000$

$-0.686567000$

3.379482000

3.690115000

3.802816000

3.691145000
$-1.172468000$

1.214210000

1.012246000

$-1.370153000$

$-0.280040000$

$-2.018455000$

$-2.374953000$

$-0.437729000$

1.860291000

2.213801000

$-0.546997000$

$-0.003294000$

$-1.545105000$

$-0.005303000$
0.000064000

$-0.000191000$

$-0.000063000$

0.000144000

0.000070000

0.000221000

0.000272000

0.000052000

$-0.000053000$

$-0.000269000$

0.000157000

0.889067000

0.001513000

$-0.889624000$
$\mathrm{TS}_{16}$

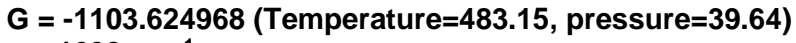

$\mathrm{v}=1638 \mathrm{~cm}^{-1}$

$E=-1103.79133013$

$\begin{array}{ll}-0.889812000 & 0.234736000\end{array}$

$8-0.727009000 \quad 1.596432000$

$\begin{array}{lll}19 & 1.565295000 & 2.619999000\end{array}$

$\begin{array}{lll}6 & -2.183274000 & -0.323802000\end{array}$

$6 \quad 0.395581000$

$6 \quad 1.091450000$

0.917315000

2.294389000

2.123490000

2.813141000

0.683454000

0.388709000

2.823429000

2.523739000

3.751659000

$-1.132537000$

$-0.998067000$

$-2.097640000$

$-3.061434000$

$-1.344415000$

$-1.877751000$

$-3.369697000$

$-3.405145000$

$-4.296396000$

$-3.320039000$

$-2.341295000$

$-2.383198000$

$-1.525109000$

$-3.273302000$

$-0.529737000$

$-0.253294000$

$-1.444933000$

$-0.890908000$

$-2.080239000$

$-1.805716000$

0.450070000

$-1.665065000$

$-0.675467000$

$-2.787686000$

$-2.300507000$

$-0.119902000$

0.791776000

$-0.131066000$

$-0.389159000$

$-0.777110000$

0.904950000

0.463587000

0.406122000

0.042702000

1.511275000

$-1.805176000$

$-1.969624000$

$-2.400642000$

$-2.173884000$

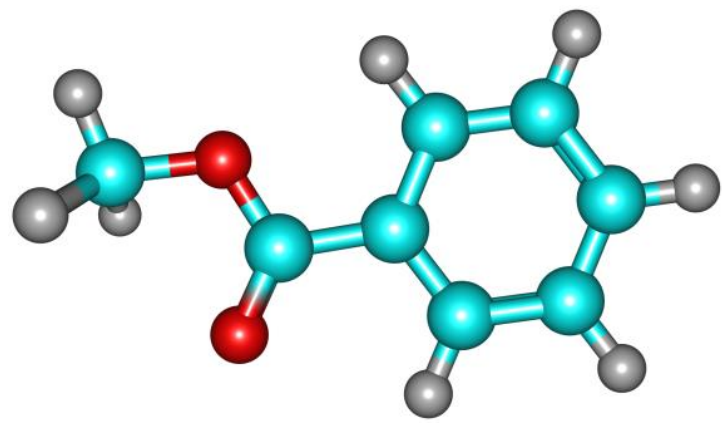

ester

$\mathrm{G}=-\mathbf{4 6 0 . 0 7 6 2 3 1}($ Temperature $=483.15$, pressure $=39.64)$

$E=-460.162403564$

23000

1.718581000

0.385633000

1.491242000

0.122548000

$-0.000032000$

0.000294000

1.960277000

$-0.726132000$
$-0.479334000$

$-0.425958000$

0.147820000

$-0.236669000$

0.939857000

$-0.873173000$

297927000

059910000

2.123785000

$-1.585537000$

$-1.708074000$

$-1.625032000$

1.662877000

2.100873000

2.111225000

1.919285000

403202000

$-0.015823000$

$-0.115195000$

$-0.184537000$

$-1.263622000$

0.222997000

0.242287000

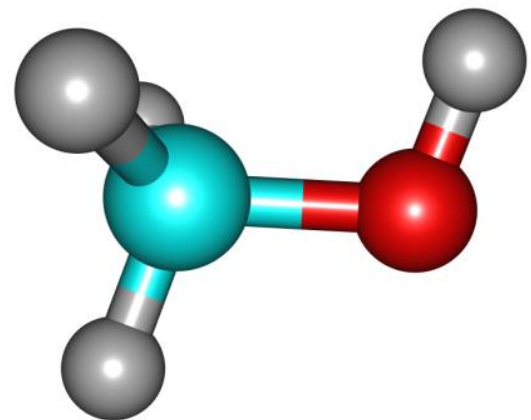

$G=\mathbf{- 1 1 5 . 7 1 7 6 8 1}($ Temperature $=483.15$, pressure $=39.64)$

$E=-115.734654366$

$\begin{array}{llll}1 & -1.140998000 & 0.752565000 & -0.000018000\end{array}$

$\begin{array}{llll}8 & -0.746704000 & -0.123352000 & 0.000016000\end{array}$

$\begin{array}{llll}6 & 0.665196000 & 0.019794000 & 0.000000000\end{array}$

$\begin{array}{lll}1.015804000 & 0.545999000 & -0.889371000\end{array}$

$\begin{array}{lll}1.015828000 & 0.549793000 & 0.887114000\end{array}$

$\begin{array}{lll}1.091819000 & -0.980299000 & 0.002147000\end{array}$

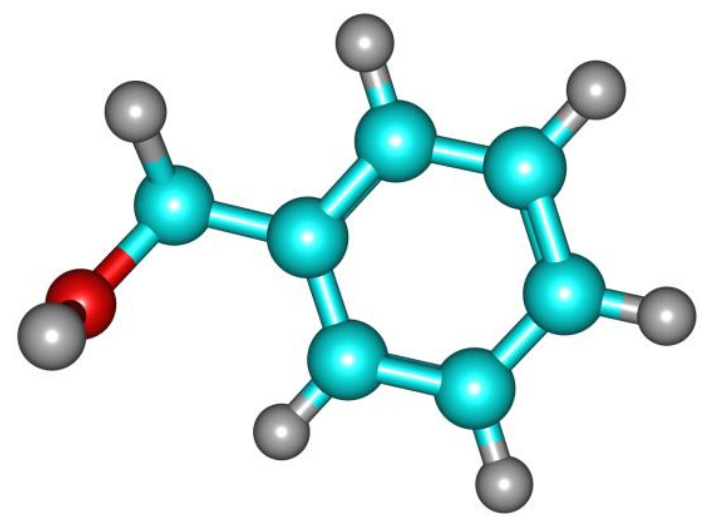

$\mathbf{G}=\mathbf{- 3 4 6 . 7 2 0 9 2 1}($ Temperature $=483.15$, pressure $=39.64)$ $E=-346.801680687$

$6-1.906954000$

$-2.768815000$

0.601312000

$-0.181443000$

$-2.625170000$

$-0.470139000$

0.149027000

$-2.118954000$

$-0.706531000$

1.070710000

$-0.439478000$

.475280000

0.261557000

0.438978000

0.495028000

1.289346000

0.006766000

$-1.054511000$

1.365663000

$-1.338412000$

1.851442000

1.008140000

2.291501000

$-0.309692000$

0.154468000

2.316950000

$-0.080598000$

0.021210000

$-0.111615000$

$-0.043399000$

0.083280000

0.052304000

2.565532000

1.816241000

0.055782000

3.348229000

$-0.531501000$

0.163842000

1.699937000

$-2.366640000$

0.106373000

$-0.710288000$

$-1.859928000$

$-0.066267000$

$-2.147034000$

0.870803000

$-0.189405000$

$-1.210667000$ 


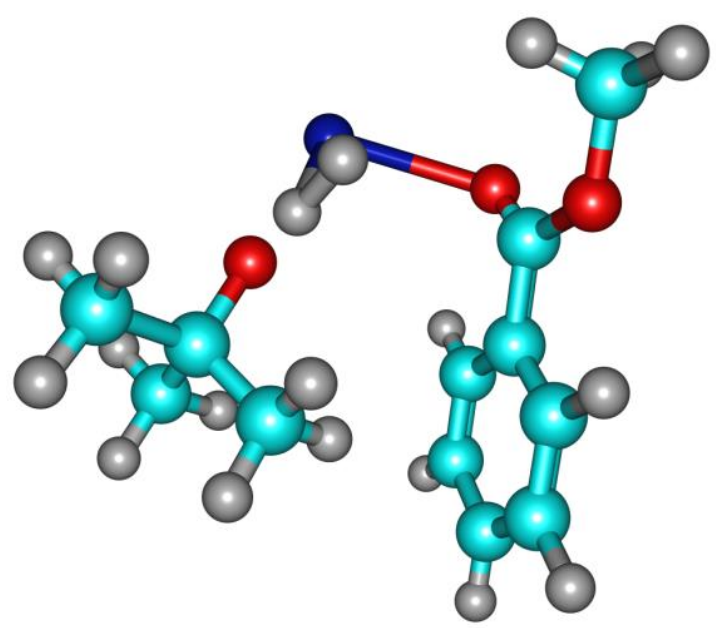

$\mathrm{TS}_{17}$

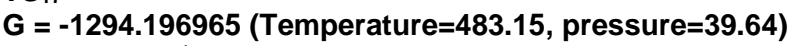

$\mathrm{v}=1538 \mathrm{~cm}^{-1}$

$E=-1294.38663743$

$\begin{array}{llll}6 & -1.961941000 & -0.669737000 & -0.014100000\end{array}$

$8 \quad-1.747227000 \quad-1.517225000 \quad-0.858736000$

$\begin{array}{llll}19 & 0.576122000 & -2.620774000 & -1.172805000\end{array}$

$\begin{array}{llll}8 & 1.885217000 & -0.846878000 & -0.061466000\end{array}$

$\begin{array}{llll}1 & 1.121163000 & -1.336983000 & 0.814977000\end{array}$

$\begin{array}{llll}1 & 0.470417000 & -1.816470000 & 1.392989000\end{array}$

$\begin{array}{llll}6 & -1.618161000 & 0.764483000 & -0.181445000\end{array}$

$\begin{array}{llll}8 & -2.549155000 & -0.941217000 & 1.137355000\end{array}$

$\begin{array}{llll}6 & 2.673878000 & 0.180594000 & 0.481669000\end{array}$

$\begin{array}{llll}6 & -1.815375000 & 1.684004000 & 0.844908000\end{array}$

$\begin{array}{llll}6 & -1.081632000 & 1.177627000 & -1.397639000\end{array}$

$\begin{array}{llll}6 & -0.749698000 & 2.508423000 & -1.590197000\end{array}$

$\begin{array}{llll}6 & -1.478696000 & 3.014138000 & 0.649222000\end{array}$

$\begin{array}{llll}6 & -0.949151000 & 3.426662000 & -0.566671000\end{array}$

$\begin{array}{llll}1 & -2.221986000 & 1.358609000 & 1.790648000\end{array}$

$\begin{array}{llll}1 & -1.627136000 & 3.728748000 & 1.446146000\end{array}$

$\begin{array}{llll}1 & -0.687575000 & 4.465052000 & -0.715885000\end{array}$

$\begin{array}{llll}1 & -0.334276000 & 2.829691000 & -2.534679000\end{array}$

$\begin{array}{llll}1 & -0.934431000 & 0.455344000 & -2.187913000\end{array}$

$\begin{array}{llll}6 & -2.880766000 & -2.312461000 & 1.377482000\end{array}$

$\begin{array}{llll}6 & 1.781477000 & 1.239040000 & 1.137597000\end{array}$

$\begin{array}{lllll}6 & 3.632232000 & -0.397580000 & 1.526637000\end{array}$

$\begin{array}{llll}6 & 3.475171000 & 0.827724000 & -0.646041000\end{array}$

$\begin{array}{llll}1 & -1.974422000 & -2.913686000 & 1.399774000\end{array}$

$\begin{array}{llll}1 & 4.268528000 & -1.157782000 & 1.070802000\end{array}$

$\begin{array}{llll}1 & 1.182202000 & 0.793707000 & 1.932743000\end{array}$

$\begin{array}{llll}1 & 2.797420000 & 1.238039000 & -1.396678000\end{array}$

$1 \quad 4.107326000 \quad 1.636365000$

$4.113787000 \quad 0.086476000$

$4.272578000 \quad 0.374051000$

$3.069097000-0.865737000$

$2.373661000 \quad 2.049195000$

$1.104282000 \quad 1.663476000$

$-3.366495000-2.331151000$

$-3.555191000$

$-1.128953000$

1.957324000

2.335070000

1.567113000

0.395632000

2.346293000
25

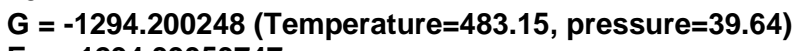

$E=-1294.39353747$

$6-1.985432000$

$\begin{array}{llll}8 & -1.781886000 & -1.154290000 & -0.985580000\end{array}$

$\begin{array}{llll}19 & 0.030526000 & -2.986654000 & -0.706942000\end{array}$

$8 \quad 1.519292000-0.995493000-0.005263000$

$\begin{array}{llll}1 & 0.976498000 & -1.232454000 & 0.801846000\end{array}$

$1 \quad-0.046657000 \quad-1.959455000 \quad 1.709282000$

$\begin{array}{llll}6 & -1.371253000 & 1.065544000 & -0.214167000\end{array}$

$\begin{array}{llll}8 & -2.811663000 & -0.444415000 & 0.858454000\end{array}$

$\begin{array}{llll}6 & 2.636424000 & -0.197583000 & 0.392345000\end{array}$

$\begin{array}{llll}6 & -1.503269000 & 1.964124000 & 0.840922000\end{array}$

$\begin{array}{llll}6 & -0.657696000 & 1.424020000 & -1.354121000\end{array}$

$\begin{array}{llll}6 & -0.094077000 & 2.686035000 & -1.447221000\end{array}$

$\begin{array}{llll}6 & -0.930130000 & 3.222783000 & 0.747009000\end{array}$

$\begin{array}{llll}6 & -0.232143000 & 3.586082000 & -0.397466000\end{array}$

$\begin{array}{lll}-2.045157000 & 1.677258000 & 1.729608000\end{array}$

$\begin{array}{lll}-1.027591000 & 3.919806000 & 1.567104000\end{array}$

$\begin{array}{lll}0.209971000 & 4.570132000 & -0.469303000\end{array}$

$\begin{array}{lll}0.454057000 & 2.967759000 & -2.335109000\end{array}$

$\begin{array}{lll}-0.555374000 & 0.713875000 & -2.162288000\end{array}$

$\begin{array}{lll}-3.422983000 & -1.730202000 & 0.994030000\end{array}$

$\begin{array}{lll}2.142848000 & 0.966469000 & 1.242529000\end{array}$

$\begin{array}{lll}3.612916000 & -1.061088000 & 1.183022000\end{array}$

$\begin{array}{lll}3.279065000 & 0.310450000 & -0.887204000\end{array}$

$\begin{array}{lll}-2.660755000 & -2.479514000 & 1.198151000\end{array}$

$\begin{array}{lll}3.953608000 & -1.900607000 & 0.576303000\end{array}$

$\begin{array}{lll}1.630501000 & 0.596978000 & 2.132002000\end{array}$

$\begin{array}{lll}2.565868000 & 0.910104000 & -1.454030000\end{array}$

$\begin{array}{lll}4.147754000 & 0.928435000 & -0.661059000\end{array}$

$\begin{array}{lll}3.603329000 & -0.523999000 & -1.509775000\end{array}$

$\begin{array}{lll}4.484296000 & -0.482236000 & 1.490279000\end{array}$

$\begin{array}{lll}3.128542000 & -1.455166000 & 2.077126000\end{array}$

$\begin{array}{lll}2.976025000 & 1.593842000 & 1.560514000\end{array}$

$\begin{array}{lll}1.444681000 & 1.579311000 & 0.672457000\end{array}$

$-4.095821000 \quad-1.647608000 \quad 1.840004000$

$\begin{array}{lll}-3.975314000 & -1.983214000 & 0.092600000\end{array}$

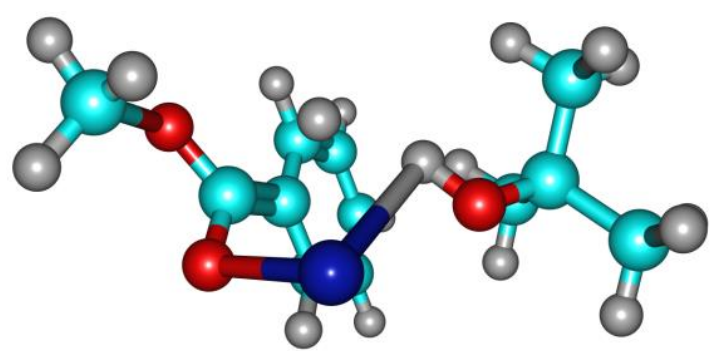

$\mathrm{TS}_{18}$

0.606877000

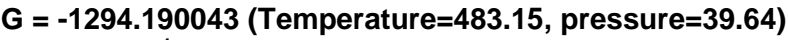

$\mathrm{v}=723 \mathrm{~cm}^{-1}$

$E=-1294.38336760$

$6 \quad-1.948462000-0.460781000 \quad-0.038052000$

$\begin{array}{llll}8 & -1.915624000 & -1.246950000 & -0.986297000\end{array}$

$\begin{array}{llll}19 & 0.079365000 & -2.797203000 & -0.808033000\end{array}$

$\begin{array}{llll}8 & 1.783348000 & -1.027905000 & -0.011736000\end{array}$

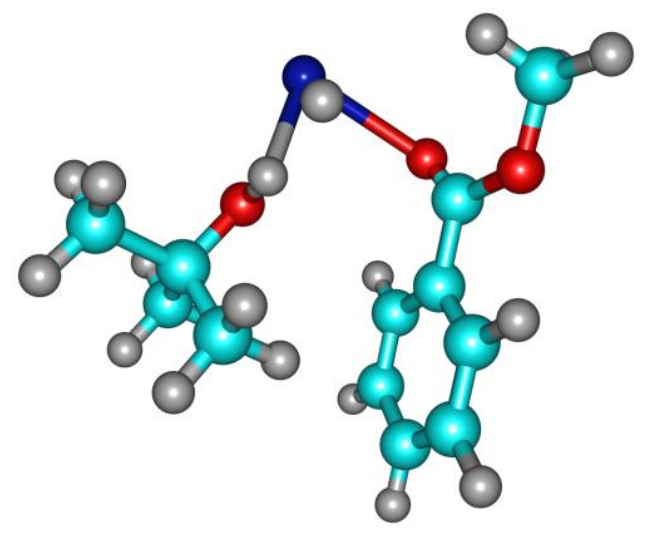

$\begin{array}{llll}1 & 0.981053000 & -0.863369000 & 0.551639000\end{array}$

$\begin{array}{llll}1 & -0.478708000 & -1.108161000 & 1.087615000\end{array}$

$\begin{array}{llll}6 & -1.431894000 & 0.940985000 & -0.142018000\end{array}$

$\begin{array}{llll}8 & -2.859507000 & -0.584776000 & 0.946824000\end{array}$

$\begin{array}{lll}-2.859507000 & -0.584776000 & 0.946824000 \\ 2.830196000 & -0.129574000 & 0.369676000\end{array}$

$\begin{array}{lll}-1.445571000 & 1.807244000 & 0.948540000\end{array}$

$\begin{array}{lll}-0.967955000 & 1.387649000 & -1.372341000\end{array}$

$\begin{array}{lll}-0.523133000 & 2.695679000 & -1.516709000\end{array}$

$\begin{array}{lll}-0.999033000 & 3.109750000 & 0.803263000\end{array}$

$\begin{array}{lll}-0.537534000 & 3.557794000 & -0.430997000\end{array}$

$\begin{array}{lll}-1.792387000 & 1.451806000 & 1.908255000\end{array}$

$\begin{array}{lll}-1.007462000 & 3.778431000 & 1.652760000\end{array}$

$\begin{array}{lll}-0.189481000 & 4.575497000 & -0.541399000\end{array}$

$\begin{array}{lll}-0.164414000 & 3.038302000 & -2.477415000\end{array}$

$\begin{array}{lll}-0.964944000 & 0.711050000 & -2.215079000\end{array}$

$\begin{array}{lll}-3.353211000 & -1.897310000 & 1.157517000\end{array}$

$\begin{array}{lll}2.406538000 & 1.294404000 & 0.034691000\end{array}$ 


$\begin{array}{rrr}3.091032000 & -0.273366000 & 1.863795000 \\ 4.053319000 & -0.533866000 & -0.434861000 \\ -2.524577000 & -2.557444000 & 1.426400000 \\ 3.360446000 & -1.301855000 & 2.104431000 \\ 1.485136000 & 1.546527000 & 0.563460000 \\ 3.844421000 & -0.466451000 & -1.502703000 \\ 4.893569000 & 0.121315000 & -0.207085000 \\ 4.342249000 & -1.558700000 & -0.201110000 \\ 3.903381000 & 0.381147000 & 2.179489000 \\ 2.197106000 & -0.009159000 & 2.430736000 \\ 3.176982000 & 2.008988000 & 0.325600000 \\ 2.228077000 & 1.393347000 & -1.035874000 \\ -4.051946000 & -1.831937000 & 1.985293000 \\ -3.857920000 & -2.277074000 & 0.272253000\end{array}$

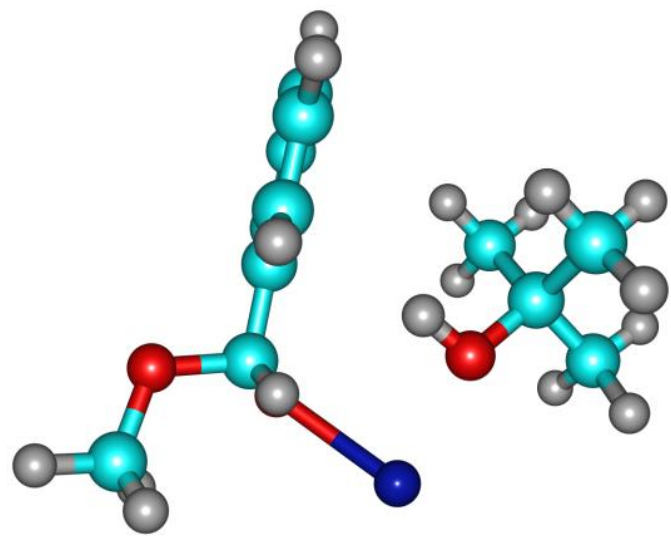

26

$\mathrm{G}=-\mathbf{1 2 9 4 . 2 2 6 6 1 3}($ Temperature $=483.15$, pressure $=39.64)$

$E=-1294.42813223$

$\mathrm{v}=10 \mathrm{~cm}^{-1}$

$\begin{array}{lrrr}6 & -1.762181000 & -0.589024000 & 0.114799000 \\ 8 & -1.545884000 & -1.336465000 & -0.956681000 \\ 19 & 0.334830000 & -2.840434000 & -0.383341000 \\ 8 & 1.562223000 & -0.762481000 & 0.683286000 \\ 1 & 0.911581000 & -0.091757000 & 0.927208000 \\ 1 & -1.265268000 & -0.986003000 & 1.040060000 \\ 6 & -1.335798000 & 0.874761000 & -0.012128000 \\ 8 & -3.166517000 & -0.507527000 & 0.503963000 \\ 6 & 2.725450000 & -0.080135000 & 0.173649000 \\ 6 & -0.933723000 & 1.594914000 & 1.108065000 \\ 6 & -1.332311000 & 1.502550000 & -1.252188000 \\ 6 & -0.939343000 & 2.828299000 & -1.370338000 \\ 6 & -0.527325000 & 2.919914000 & 0.994382000 \\ 6 & -0.531515000 & 3.540112000 & -0.247670000 \\ 1 & -0.937939000 & 1.112382000 & 2.079604000 \\ 1 & -0.209580000 & 3.465875000 & 1.872473000 \\ 1 & -0.215791000 & 4.570020000 & -0.342054000 \\ 1 & -0.943129000 & 3.307535000 & -2.340179000 \\ 1 & -1.632360000 & 0.937499000 & -2.123883000 \\ 6 & -3.704346000 & -1.773530000 & 0.797966000 \\ 6 & 2.300484000 & 0.840787000 & -0.959967000 \\ 6 & 3.363344000 & 0.711343000 & 1.305427000 \\ 6 & 3.657218000 & -1.170748000 & -0.322401000 \\ 1 & -3.106865000 & -2.287130000 & 1.561479000 \\ 1 & 3.650989000 & 0.047502000 & 2.120354000 \\ 1 & 1.615051000 & 1.606747000 & -0.591684000 \\ 1 & 3.216553000 & -1.709234000 & -1.163227000 \\ 1 & 4.595238000 & -0.737393000 & -0.665974000 \\ 1 & 3.878911000 & -1.877530000 & 0.477193000 \\ 1 & 4.250691000 & 1.237765000 & 0.955377000 \\ 1 & 2.659084000 & 1.450384000 & 1.691471000 \\ 1 & 3.167069000 & 1.338821000 & -1.394424000 \\ 1 & 1.795608000 & 0.275015000 & -1.743835000 \\ 1 & -4.709873000 & -1.625523000 & 1.188188000 \\ 1 & -3.760032000 & -2.410404000 & -0.086785000\end{array}$
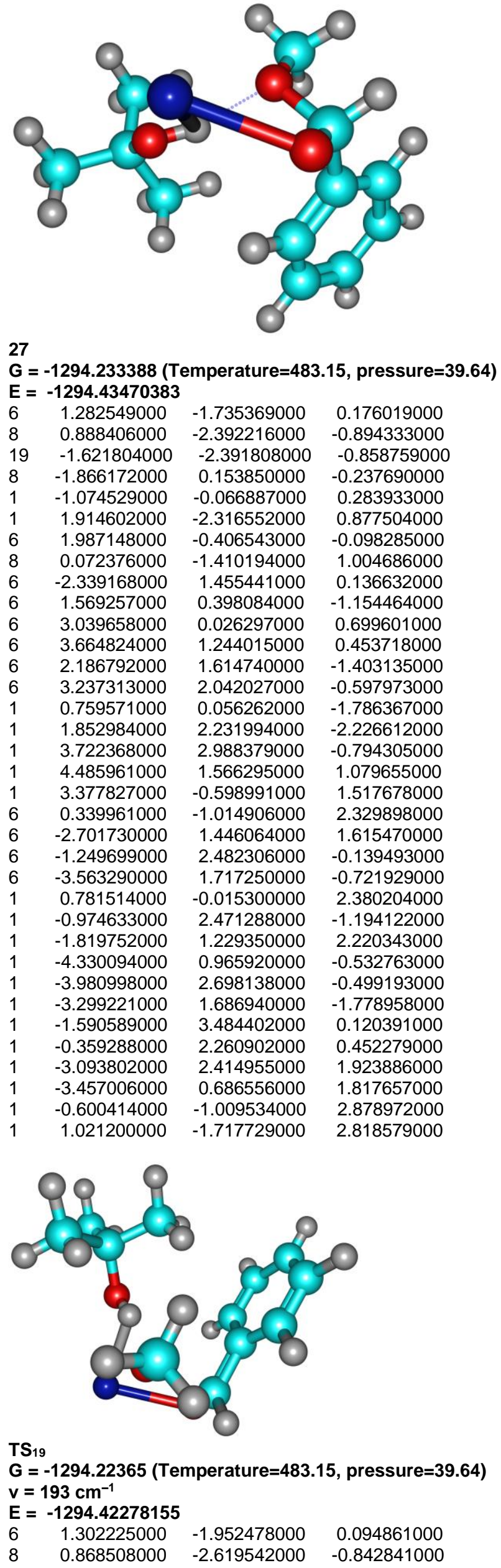


\begin{tabular}{lrrr}
19 & -1.694954000 & -2.318171000 & -0.815457000 \\
8 & -1.635105000 & 0.301512000 & -0.467231000 \\
1 & -1.123375000 & -0.183717000 & 0.261877000 \\
1 & 1.544826000 & -2.425788000 & 1.053388000 \\
6 & 1.886702000 & -0.599079000 & -0.099523000 \\
8 & -0.480980000 & -1.302377000 & 1.152744000 \\
6 & -1.940815000 & 1.619080000 & -0.020720000 \\
6 & 1.780653000 & 0.050002000 & -1.326655000 \\
6 & 2.586376000 & 0.001296000 & 0.941831000 \\
6 & 3.164957000 & 1.250535000 & 0.764945000 \\
6 & 2.365231000 & 1.292720000 & -1.506101000 \\
6 & 3.053336000 & 1.896552000 & -0.459164000 \\
1 & 1.245655000 & -0.429431000 & -2.135046000 \\
1 & 2.282206000 & 1.796420000 & -2.459268000 \\
1 & 3.506985000 & 2.867908000 & -0.600445000 \\
1 & 3.706730000 & 1.715999000 & 1.576442000 \\
1 & 2.680937000 & -0.516625000 & 1.887617000 \\
6 & -0.176421000 & -0.923372000 & 2.447554000 \\
6 & -2.732986000 & 1.540515000 & 1.280753000 \\
6 & -0.641250000 & 2.387911000 & 0.196811000 \\
6 & -2.771456000 & 2.274571000 & -1.111608000 \\
1 & 0.205198000 & 0.107835000 & 2.520197000 \\
1 & -0.109274000 & 2.503619000 & -0.746708000 \\
1 & -2.138265000 & 1.060826000 & 2.059659000 \\
1 & -3.701064000 & 1.725540000 & -1.264714000 \\
1 & -3.018516000 & 3.302010000 & -0.845165000 \\
1 & -2.218018000 & 2.285854000 & -2.050934000 \\
1 & -0.834897000 & 3.378763000 & 0.609004000 \\
1 & 0.006533000 & 1.846023000 & 0.890467000 \\
1 & -3.011221000 & 2.535432000 & 1.629047000 \\
1 & -3.643973000 & 0.959192000 & 1.135073000 \\
1 & -1.048922000 & -0.981410000 & 3.114458000 \\
1 & 0.593895000 & -1.569453000 & 2.899461000 \\
& & & \\
\hline & &
\end{tabular}

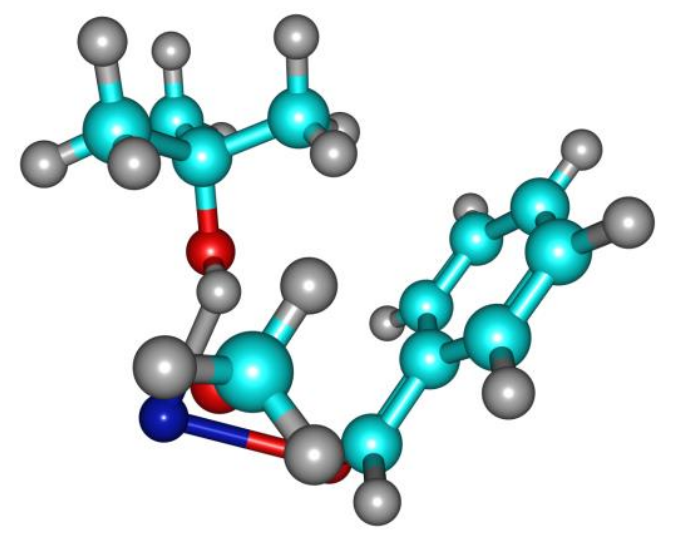

28

$G=-1294.230172($ Temperature $=483.15$, pressure $=39.64)$ $E=-1294.42413968$

$\begin{array}{llll}8 & 0.582680000 & -2.602390000 & -1.021901000\end{array}$

$19 \quad-1.995631000 \quad-2.139866000 \quad-0.766987000$

$\begin{array}{llll}8 & -1.501621000 & 0.443768000 & -0.493846000\end{array}$

$\begin{array}{llll}1 & -1.197641000 & -0.115608000 & 0.321219000\end{array}$

$\begin{array}{llll}1 & 1.202608000 & -2.599508000 & 0.892722000\end{array}$

$\begin{array}{llll}6 & 1.869023000 & -0.802546000 & -0.152657000\end{array}$

$\begin{array}{llll}8 & -0.926280000 & -1.229569000 & 1.280310000\end{array}$

$\begin{array}{llll}6 & -1.700490000 & 1.791387000 & -0.089481000\end{array}$

$\begin{array}{llll}6 & 1.918166000 & -0.082444000 & -1.345020000\end{array}$

$\begin{array}{llll}6 & 2.539470000 & -0.336243000 & 0.973800000\end{array}$

$\begin{array}{llll}6 & 3.252634000 & 0.852400000 & 0.913697000\end{array}$

$\begin{array}{llll}6 & 2.638800000 & 1.096566000 & -1.406465000\end{array}$

$\begin{array}{llll}6 & 3.302304000 & 1.565490000 & -0.276416000\end{array}$

$\begin{array}{llll}1 & 1.393251000 & -0.456429000 & -2.213215000\end{array}$

$\begin{array}{llll}1 & 2.680887000 & 1.658206000 & -2.329098000\end{array}$

$\begin{array}{llll}1 & 3.861182000 & 2.489835000 & -0.326604000\end{array}$

$\begin{array}{llll}1 & 3.771634000 & 1.218537000 & 1.788111000\end{array}$

$\begin{array}{llll}1 & 2.501392000 & -0.909049000 & 1.891222000\end{array}$

$\begin{array}{llll}6 & -0.423732000 & -0.868261000 & 2.514107000\end{array}$

$\begin{array}{llll}6 & -2.574877000 & 1.826914000 & 1.161550000\end{array}$

$\begin{array}{llll}6 & -0.346743000 & 2.434701000 & 0.198980000\end{array}$
$6 \quad-2.391369000$

$1 \quad 0.085871000$

0.250518000

$-2.078693000$

$-3.365661000$

$-2.538869000$

$-1.788031000$

$-0.463796000$

0.199571000

$-2.777838000$

$-3.526908000$

$-1.202335000$

0.317386000

$2.509316000-1.238437000$

$0.110435000 \quad 2.508629000$

$2.478583000-0.711120000$

$1.317252000 \quad 1.988924000$

$2.061353000-1.437000000$

$3.564081000-1.006310000$

$2.437514000-2.143929000$

$3.448263000 \quad 0.584714000$

$1.844527000 \quad 0.938483000$

$2.853916000 \quad 1.466306000$

$\begin{array}{ll}1.329306000 & 0.972944000\end{array}$

$-0.800957000 \quad 3.291543000$

$-1.590432000 \quad 2.897264000$

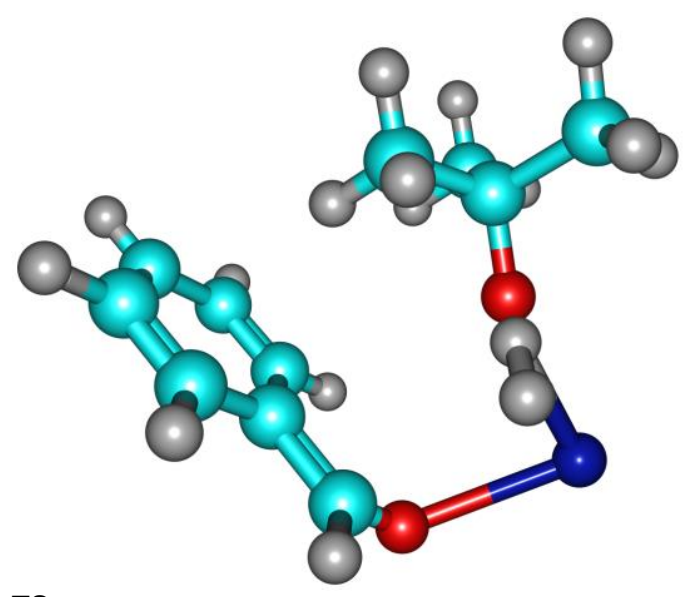

$\mathrm{TS}_{20}$

$\mathrm{G}=-\mathbf{1 1 7 9 . 6 5 4 1 0 1}$ (Temperature $=483.15$, pressure $=39.64$ )

$\mathrm{v}=1553 \mathrm{~cm}^{-1}$

$E=-1179.81739892$

$\begin{array}{llll}6 & 0.802808000 & -2.164134000 & -0.558535000\end{array}$

$\begin{array}{llll}8 & 0.055548000 & -2.736432000 & 0.210323000\end{array}$

$\begin{array}{llll}19 & -2.447978000 & -2.005196000 & 0.229358000\end{array}$

$\begin{array}{llll}8 & -1.604907000 & 0.312144000 & -0.006265000\end{array}$

$\begin{array}{llll}1 & -1.446582000 & -0.241603000 & -1.133927000\end{array}$

$\begin{array}{llll}1 & -1.374747000 & -0.806935000 & -1.942935000\end{array}$

$\begin{array}{llll}6 & 1.669983000 & -1.038395000 & -0.189288000\end{array}$

$\begin{array}{llll}6 & -1.491090000 & 1.708426000 & -0.092281000\end{array}$

$\begin{array}{llll}6 & 2.474000000 & -0.471734000 & -1.173269000\end{array}$

$\begin{array}{llll}6 & 1.690401000 & -0.539870000 & 1.113115000\end{array}$

$\begin{array}{llll}6 & 2.527831000 & 0.514367000 & 1.426851000\end{array}$

$\begin{array}{llll}6 & 3.309815000 & 0.589163000 & -0.857429000\end{array}$

$\begin{array}{llll}6 & 3.337411000 & 1.076338000 & 0.441889000\end{array}$

$\begin{array}{llll}1 & 2.436259000 & -0.863473000 & -2.181916000\end{array}$

$1 \quad 3.934564000 \quad 1.034403000 \quad-1.618345000$

$\begin{array}{llll}1 & 3.988475000 & 1.902749000 & 0.691499000\end{array}$

$1 \quad 2.552030000 \quad 0.906789000 \quad 2.433572000$

$\begin{array}{llll}1 & 1.051028000 & -0.984046000 & 1.863442000\end{array}$

$\begin{array}{llll}6 & -0.227696000 & 2.091566000 & -0.869494000\end{array}$

$\begin{array}{llll}6 & -2.719742000 & 2.286851000 & -0.800081000\end{array}$

$\begin{array}{llll}6 & -1.400468000 & 2.276583000 & 1.322723000\end{array}$

$\begin{array}{lll}-3.626161000 & 2.020936000 & -0.253721000\end{array}$

$\begin{array}{lll}-0.257685000 & 1.678871000 & -1.878630000\end{array}$

$\begin{array}{lll}-0.531699000 & 1.858989000 & 1.834960000\end{array}$

$\begin{array}{lll}-1.305543000 & 3.363953000 & 1.313895000\end{array}$

$\begin{array}{lll}-2.292789000 & 2.014485000 & 1.893415000\end{array}$

$\begin{array}{lll}-2.670357000 & 3.374749000 & -0.872589000\end{array}$

$\begin{array}{lll}-2.797950000 & 1.879708000 & -1.809219000\end{array}$

$\begin{array}{lll}-0.121745000 & 3.175088000 & -0.946096000\end{array}$

$\begin{array}{lll}0.653507000 & 1.695523000 & -0.363837000\end{array}$

$0.876324000-2.492123000-1.605053000$ 


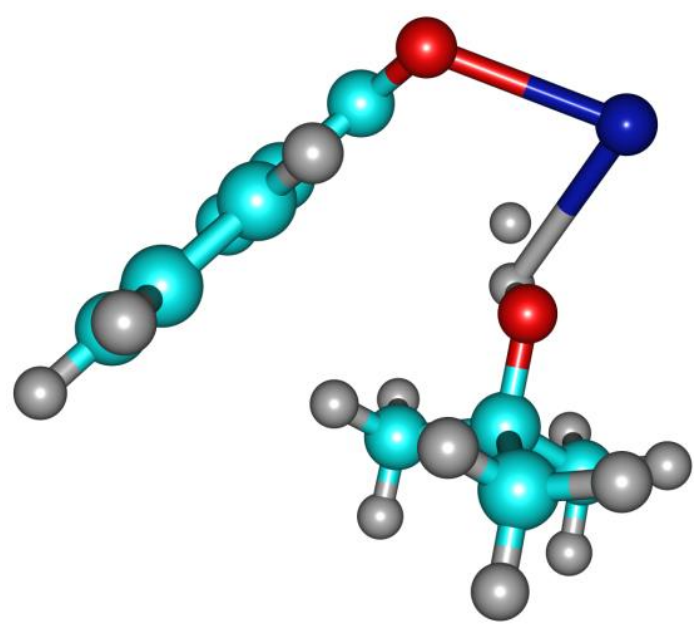

29

$\mathrm{G}=\mathbf{- 1 1 7 9 . 6 5 8 0 4}($ Temperature $=483.15$, pressure $=39.64)$

$E=\mathbf{- 1 1 7 9 . 8 2 4 6 7 5 8 4}$

$6-0.694703000$

$\begin{array}{ll}0.694703000 & 2.176768000\end{array}$

$\begin{array}{lll}8 & 0.059071000 & 2.697082000\end{array}$

$\begin{array}{lll}19 & 2.560574000 & 1.982973000\end{array}$

$8 \quad 1.566534000$

1.512971000

1.627637000

$-0.392831000$

$-0.010261000$

0.984751000

1.088555000

$-1.624279000$

1.405357000

$-2.419784000$

$-1.713682000$

$-2.608801000$

$-3.313767000$

$-3.408877000$

$-2.329599000$

$-3.932523000$

$-4.106247000$

$-2.686898000$

$-1.082262000$

0.113236000

2.602164000

1.335966000

3.526772000

0.140391000

0.492295000

1.209469000

2.250911000

2.516077000

2.664425000

$-0.030125000$

$-1.811833000$

0.560323000

0.591457000

$-0.423176000$

$-0.461138000$

$-0.947039000$

0.950463000

$-0.876357000$

$-1.741913000$

$-0.812548000$

1.007658000

$-2.139870000$

$-2.420869000$

$-2.297919000$

$-2.170853000$

$-1.732657000$

$-1.834939000$

$-3.379765000$

$-2.041663000$

$-3.506677000$

$-2.035560000$

$-3.218663000$

$-0.739831000-1.709064000$

2.525992000

$-0.514165000$

0.285546000

0.183023000

$-0.013772000$

$-0.937960000$

$-2.109786000$

$-0.182212000$

$-0.075894000$

$-1.194028000$

1.118193000

1.401796000

$-0.908207000$

0.388479000

$-2.199877000$

$-1.690666000$

0.614252000

2.407023000

1.891134000

$-0.814852000$

$-0.797597000$

1.361757000

$-0.276679000$

$-1.826696000$

1.875214000

1.395279000

1.896645000

$-0.845371000$

$-1.815951000$

$-0.881836000$

$-0.291008000$

$-1.554968000$

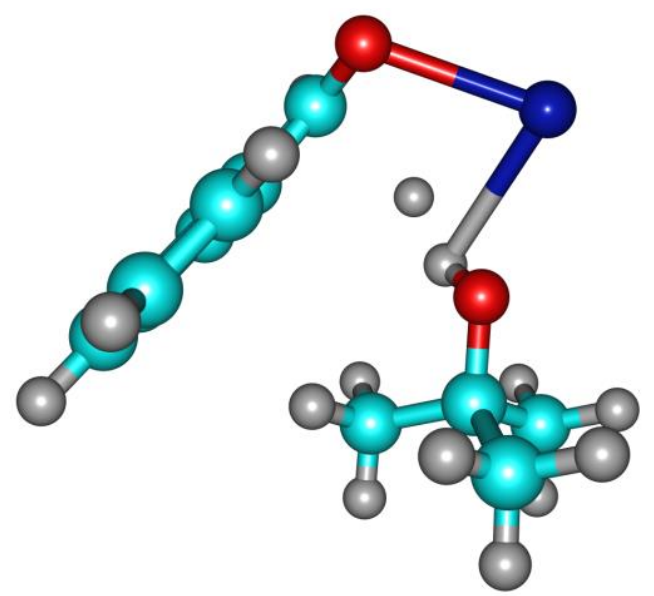

$\mathrm{TS}_{21}$

$G=-1179.652984($ Temperature $=483.15$, pressure $=39.64)$

$\mathrm{v}=552 \mathrm{~cm}^{-1}$

$E=-1179.82201028$
$6-0.911986000$

$8-0.328361000$

$19 \quad 2.162229000$

1.565515000

1.150955000

0.677966000

$-1.666013000$

1.648593000

$-2.374111000$

$-1.734627000$

$-2.512248000$

$-3.146994000$

$-3.218751000$

$-2.306855000$

$-3.694491000$

$-3.824574000$

$-2.570183000$

$-1.186744000$

0.336869000

2.809121000

1.887684000

3.745003000

0.151609000

1.056896000

1.980219000

2.804800000

2.905975000

2.648058000

0.367357000

$-0.493162000$

$-1.097645000$

2.141856000 2.817449000

2.255359000

$-0.281475000$

0.130404000

1.169637000

0.910333000

$-1.697795000$

0.243534000

0.466773000

$-0.635757000$

$-0.861779000$

$-1.299690000$

0.593302000

$-1.380642000$

$-2.159696000$

$-0.978220000$

0.996528000

$-2.202545000$

$-2.008209000$

$-2.300724000$

$-1.636176000$

$-1.737839000$

$-2.063948000$

$-3.384415000$

$-1.903576000$

$-3.082623000$

$-1.531861000$

$-3.283494000$

$-1.960615000$

2.537631000

$-0.561383000$

0.282672000

0.166376000

0.119081000

$-0.692905000$

$-1.692760000$

$-0.218964000$

$-0.068991000$

$-1.213811000$

1.098433000

1.417528000

$-0.896234000$

0.421416000

$-2.236679000$

$-1.670493000$

0.671465000

2.441329000

1.865628000

$-0.658065000$

$-1.007406000$

1.304728000

$-0.589626000$

$-1.627952000$

1.970175000

1.238038000

1.740749000

$-1.165444000$

$-1.975234000$

$-0.796417000$

0.005483000

$-1.564017000$

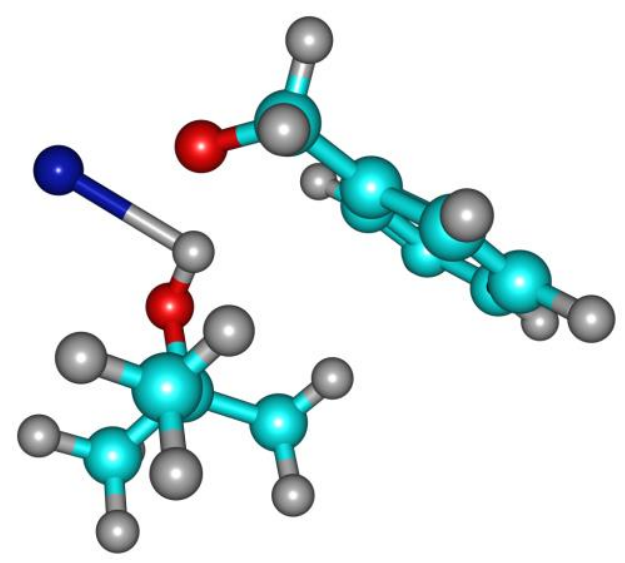

30

$\mathrm{G}=\mathbf{- 1 1 7 9 . 7 0 8 2 4}($ Temperature $=483.15$, pressure $=39.64)$

$E=-1179.88351947$

$\begin{array}{llll}8 & 0.747894000 & -1.728057000 & 0.423335000\end{array}$

$\begin{array}{llll}19 & 3.018286000 & -1.633509000 & -0.467994000\end{array}$

$8 \quad 1.452854000$

$\begin{array}{rrr}1.452854000 & 0.488090000 & -0.582498000 \\ 0.994197000 & -0.304466000 & -0.123129000\end{array}$

$\begin{array}{lll}-0.427343000 & -1.547675000 & 2.116583000\end{array}$

$\begin{array}{lll}-1.590644000 & -1.068155000 & 0.408793000\end{array}$

$\begin{array}{lll}1.193992000 & 1.709028000 & 0.102349000\end{array}$

$\begin{array}{lll}-2.544843000 & -0.405882000 & 1.175592000\end{array}$

$\begin{array}{lll}-1.675529000 & -0.976262000 & -0.978923000\end{array}$

$\begin{array}{lll}-2.687433000 & -0.244757000 & -1.583659000\end{array}$

$\begin{array}{lll}-3.559769000 & 0.330790000 & 0.576199000\end{array}$

$\begin{array}{lll}-3.633307000 & 0.415133000 & -0.807446000\end{array}$

$\begin{array}{lll}-2.483364000 & -0.454937000 & 2.256385000\end{array}$

$\begin{array}{lll}-4.285907000 & 0.847328000 & 1.189582000\end{array}$

$\begin{array}{lll}-4.416775000 & 0.993477000 & -1.277528000\end{array}$

$\begin{array}{lll}-2.735441000 & -0.181163000 & -2.662620000\end{array}$

$-0.927580000 \quad-1.470845000 \quad-1.584785000$

$\begin{array}{lll}-0.136237000 & 2.291147000 & -0.369853000\end{array}$

$\begin{array}{lll}1.147587000 & 1.456187000 & 1.605870000\end{array}$

$\begin{array}{lll}2.332361000 & 2.658327000 & -0.242525000\end{array}$

$\begin{array}{lll}2.084581000 & 1.015279000 & 1.949822000\end{array}$

$\begin{array}{lll}-0.960953000 & 1.628394000 & -0.104674000\end{array}$ 


$\begin{array}{rrrr}1 & 2.387500000 & 2.803511000 & -1.321972000 \\ 1 & 2.185927000 & 3.630704000 & 0.227442000 \\ 1 & 3.284656000 & 2.250935000 & 0.099483000 \\ 1 & 0.986081000 & 2.387274000 & 2.149574000 \\ 1 & 0.333695000 & 0.771862000 & 1.852400000 \\ 1 & -0.318961000 & 3.264646000 & 0.086915000 \\ 1 & -0.128195000 & 2.414534000 & -1.453441000 \\ 1 & -0.793059000 & -2.916539000 & 1.088533000\end{array}$

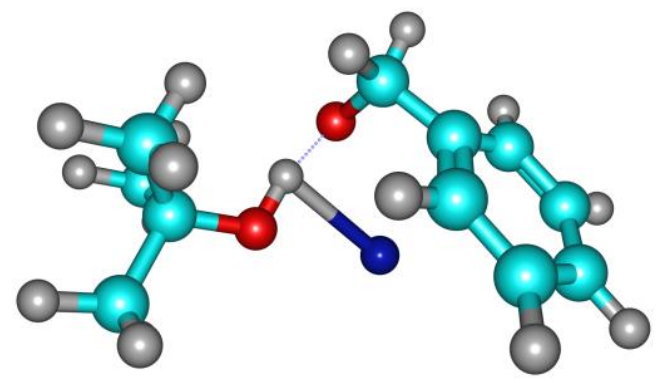

31

$\mathbf{G}=\mathbf{- 1 1 7 9 . 7 1 2 8 9 6}($ Temperature $=483.15$, pressure $=39.64)$

$E=\mathbf{- 1 1 7 9 . 8 8 7 7 2 7 4 6}$

$\begin{array}{llll}6 & 0.569083000 & 1.979971000 & 0.390532000\end{array}$

$\begin{array}{llll}8 & -0.218792000 & 1.624702000 & -0.702057000\end{array}$

$\begin{array}{llll}19 & 0.627479000 & -0.797061000 & -1.557825000\end{array}$

$\begin{array}{llll}8 & -1.583831000 & -0.403719000 & -0.308458000\end{array}$

$\begin{array}{llll}1 & -1.096856000 & 0.526314000 & -0.406350000\end{array}$

$\begin{array}{llll}1 & -0.011446000 & 2.026746000 & 1.325748000\end{array}$

$\begin{array}{llll}6 & 1.675102000 & 0.960527000 & 0.568888000\end{array}$

$\begin{array}{llll}6 & -2.898228000 & -0.229234000 & 0.195287000\end{array}$

$\begin{array}{llll}6 & 1.483281000 & -0.161244000 & 1.373370000\end{array}$

$\begin{array}{llll}6 & 2.813355000 & 1.016701000 & -0.236228000\end{array}$

$\begin{array}{llll}6 & 3.720729000 & -0.035039000 & -0.262488000\end{array}$

$\begin{array}{llll}6 & 2.387469000 & -1.218072000 & 1.351123000\end{array}$

$\begin{array}{llll}6 & 3.502766000 & -1.162236000 & 0.523746000\end{array}$

$\begin{array}{llll}1 & 0.601605000 & -0.216879000 & 1.999943000\end{array}$

$\begin{array}{llll}1 & 2.218414000 & -2.086156000 & 1.973495000\end{array}$

$\begin{array}{llll}1 & 4.202967000 & -1.985452000 & 0.498991000\end{array}$

$\begin{array}{lllll}1 & 4.594963000 & 0.020386000 & -0.896747000\end{array}$

$\begin{array}{llll}1 & 2.972322000 & 1.884690000 & -0.864807000\end{array}$

$\begin{array}{llll}6 & -3.457980000 & -1.612988000 & 0.490689000\end{array}$

$\begin{array}{llll}6 & -2.845809000 & 0.604877000 & 1.473003000\end{array}$

$\begin{array}{llll}6 & -3.755029000 & 0.476038000 & -0.853400000\end{array}$

$\begin{array}{llll}1 & -2.432902000 & 1.592684000 & 1.262333000\end{array}$

$\begin{array}{lll}-2.840071000 & -2.120223000 & 1.232482000\end{array}$

$\begin{array}{lll}-3.792010000 & -0.115152000 & -1.769060000\end{array}$

$\begin{array}{lll}-4.775088000 & 0.623288000 & -0.496877000\end{array}$

$\begin{array}{lll}-3.329980000 & 1.452059000 & -1.091260000\end{array}$

$\begin{array}{lll}-3.841056000 & 0.735146000 & 1.899103000\end{array}$

$\begin{array}{lll}-2.214348000 & 0.117025000 & 2.216747000\end{array}$

$\begin{array}{lll}-4.475946000 & -1.549412000 & 0.875221000\end{array}$

$\begin{array}{lll}-3.471166000 & -2.217291000 & -0.417158000\end{array}$

$\begin{array}{lll}1.021044000 & 2.972726000 & 0.253860000\end{array}$

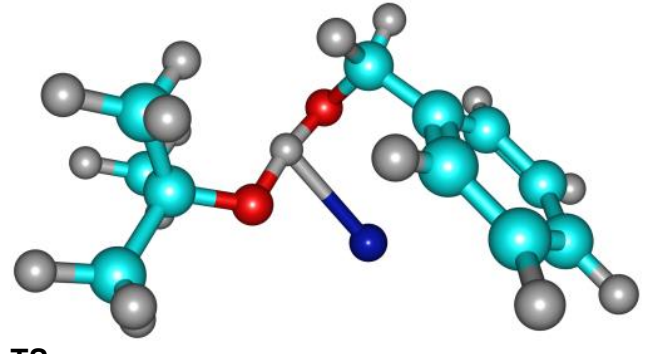

$\mathrm{TS}_{22}$

$\mathrm{G}=-\mathbf{1 1 7 9 . 7 1 4 1 2 9}($ Temperature $=483.15$, pressure $=39.64)$

$\mathrm{v}=886 \mathrm{~cm}^{-1}$

$E=-1179.88666473$

$\begin{array}{lccc}6 & 0.515806000 & 2.005334000 & 0.187719000 \\ 8 & -0.280083000 & 1.546956000 & -0.872522000 \\ 19 & 0.596846000 & -1.071200000 & -1.375019000 \\ 8 & -1.526571000 & -0.397967000 & -0.219083000 \\ 1 & -0.971007000 & 0.640120000 & -0.506063000\end{array}$

$1-0.067421000$

$6 \quad 1.645418000$

$6-2.850274000$

$6 \quad 1.526629000$

2.740581000

3.677804000

2.462671000

3.533889000

0.678367000

2.351242000

4.257912000

4.518266000

2.841594000

$-3.381925000$

$-2.893749000$

$-3.714516000$

$-2.523120000$

$-2.768763000$

$-3.700043000$

$-4.751108000$

$-3.324272000$

$-3.908548000$

$-2.265157000$

$-4.413222000$

$-3.346610000$

0.931048000
2.118483000

1.031613000

$-0.206629000$

0.033639000

$-0.021694000$

$-0.992575000$

$-1.028620000$

0.045984000

$-1.766021000$

$-1.829333000$

$-0.036507000$

1.772738000

$-1.531954000$

0.851091000

0.254325000

1.806109000

$-1.869016000$

$-0.495544000$

0.417305000

1.188644000

1.002869000

0.543971000

$-1.441016000$

$-2.295526000$

2.988821000
1.000271000
1.111021000

0.440320000

0.209167000

1.404797000

$-0.424161000$

$-0.348125000$

1.485818000

2.077488000

2.233253000

0.658346000

$-1.028452000$

$-1.176898000$

0.748353000

1.314841000

$-0.967018000$

0.938418000

1.585546000

$-1.759384000$

$-0.667745000$

$-1.373872000$

1.685629000

2.152423000

1.092321000

$-0.030175000$

$-0.055434000$
0.601920000

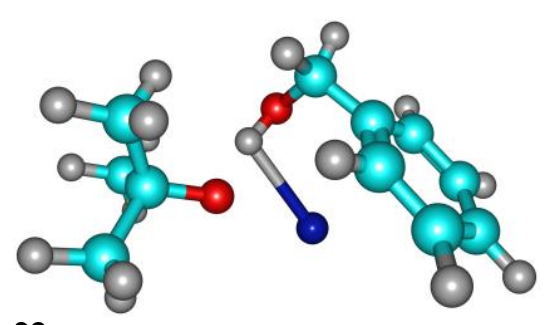

32

$\mathrm{G}=\mathbf{- 1 1 7 9 . 6 7 3 2 0 9}($ Temperature $=483.15$, pressure $=39.64)$

$E=-\mathbf{1 1 7 9 . 8 8 7 3 5 6 7 3}$

$\begin{array}{lll}6 & 0.548598000 & 2.050527000\end{array}$

$8-0.258356000$

$19 \quad 0.578354000$

1.657193000

0.217836000

$8-1.504780000$

$-1.076902000$

$-0.871512000$

$1-0.867989000$

$-0.046891000$

$-0.381628000$

0.837871000

2.159808000

1.641322000

1.034080000

$-2.813264000$

1.500287000

$-0.219746000$

0.037567000

0.978957000

$2.7304050 \mathrm{C}$

$-0.066067000$

3.642224000

$-1.010991000$

$-1.070595000$

0.068912000

0.655021000

$-1.783480000$

2.284061000

$-1.888792000$

4.182182000

$-0.099892000$

1.751401000

$-1.520602000$

2.847844000

$-3.295352000$

$-2.879929000$

0.907746000

$-1.382998000$

$-0.252201000$

$-0.569029000$

1.130037000

0.449468000

0.201646000

1.411828000

$-0.421051000$

$-0.351753000$

1.486031000

0.597376000

2.087238000

2.231743000

0.649197000

$-1.035592000$

$-1.171274000$

0.848456000

$-3.736146000$

0.130852000

1.239790000

$-0.971888000$

$-2.549719000$

1.848647000

0.795401000

$-2.646681000$

$-1.781741000$

1.686582000

$-3.711125000$

$-0.666709000$

$-1.716479000$

$-4.771294000$

0.271088000

$-0.654660000$

$-3.396463000$

$-3.891874000$

$-2.224609000$

$-4.318867000$

051968000

$-1.449191000$

1.623326000

1.050249000

2.082282000

1.219018000

$-3.258516000$

$-2.335287000$

0.123152000

0.988758000

$-0.011520000$ 